\title{
ARITHMETIC OF FUNCTION FIELD UNITS
}

\author{
BRUNO ANGLÈS AND FLORIC TAVARES RIBEIRO
}

Abstract. We prove a "discrete analogue" for Taelman's class modules of certain Conjectures formulated by R. Greenberg for cyclotomic fields.

\section{Contents}

1. Introduction

2. Stark Units

2.1. Preliminaries

2.2. $\quad \mathbb{F}_{q}(z)[\theta]$-Drinfeld modules

2.3. Integrality results

2.4. The canonical $z$-deformation of a Drinfeld module 10

2.5. Stark units for Drinfeld modules 13

2.6. An example 20

3. Deformation of Drinfeld modules over several variable Tate algebras 24

3.1. Basic properties 24

3.2. Evaluation at $z=1 \quad 28$

3.3. The case of the Carlitz module 31

3.4. $P$-adic $L$-series 40

4. Arithmetic of cyclotomic function fields 45

4.1. Dirichlet characters 45

4.2. $P$-adic Dirichlet-Goss $L$-series 47

4.3. The class module of the $P$ th cyclotomic function field 52

References 56

\section{INTRODUCTION}

Let us recall some facts from classical cyclotomic theory (we refer the reader to [30, chapters 13 and 15). Let $p \geq 3$ be an odd prime number and let $\Delta=$ $\operatorname{Gal}\left(\mathbb{Q}\left(\mu_{p}\right) / \mathbb{Q}\right)$. Let $M$ be the projective limit for the norm map of the $p$-Sylow subgroups of the ideal class groups along the cyclotomic $\mathbb{Z}_{p}$-extension of $\mathbb{Q}\left(\mu_{p}\right)$ : $\mathbb{Q}\left(\mu_{p^{\infty}}\right)$. Let $\Gamma=\operatorname{Gal}\left(\mathbb{Q}\left(\mu_{p^{\infty}}\right) / \mathbb{Q}\left(\mu_{p}\right)\right)$, and let $\gamma \in \Gamma$ such that:

$$
\forall \zeta \in \mu_{p^{\infty}}, \gamma(\zeta)=\zeta^{1+p}
$$

We can identify $\mathbb{Z}_{p}[[\Gamma]]$ with $\mathbb{Z}_{p}[[T]]$ by sending $\gamma$ to $1+T$. Therefore $M$ is a $\mathbb{Z}_{p}[[T]][\Delta]$-module. Let $\chi \in \widehat{\Delta}:=\operatorname{Hom}\left(\Delta, \mathbb{Z}_{p}^{\times}\right)$, and let $e_{\chi}=\frac{1}{p-1} \sum_{\delta \in \Delta} \chi(\delta) \delta^{-1} \in$

Date: October 8, 2018. 
$\mathbb{Z}_{p}[\Delta]$. For $\chi \in \widehat{\Delta}$, we set:

$$
M_{\chi}=e_{\chi} M \text {. }
$$

Then $M_{\chi}$ is a finitely generated and torsion $\mathbb{Z}_{p}[[T]]$-module. If $\chi$ is odd distinct from the Teichmüller character : $\omega_{p}$, B. Mazur and A. Wiles proved that the characteristic ideal of the $\mathbb{Z}_{p}[[T]]$-module $M_{\chi}$ is generated by some polynomial $P_{\chi}(T) \in \mathbb{Z}_{p}[T]$ such that there exists $U_{\chi}(T) \in \mathbb{Z}_{p}[[T]]^{\times}$with the property ([21]):

$$
\forall y \in \mathbb{Z}_{p}, P_{\chi}\left((1+p)^{y}-1\right) U_{\chi}\left((1+p)^{y}-1\right)=L_{p}\left(y, \omega_{p} \chi^{-1}\right),
$$

where $L_{p}\left(., \omega_{p} \chi^{-1}\right)$ is the $p$-adic $L$-function attached to the even character $\omega_{p} \chi^{-1}$. Observe that if Vandiver's Conjecture is true for the prime $p$, then for $\chi$ odd, $M_{\chi}$ is a cyclic $\mathbb{Z}_{p}[[T]]$-module and for $\chi$ even: $M_{\chi}=\{0\}$. R. Greenberg has conjectured weaker statements (see [17]):

Pseudo-cyclicity Conjecture: If $\chi$ is odd then there exists an injective morphism of $\mathbb{Z}_{p}[[T]]$-modules between a cyclic $\mathbb{Z}_{p}[[T]]$-module and $M_{\chi}$ such that the cokernel of this morphism is finite;

Pseudo-nullity Conjecture: If $\chi$ is even then $M_{\chi}$ is finite.

These two conjectures are open.

L. Taelman has recently introduced new arithmetic objects associated to Drinfeld modules ([26]): class modules and modules of units; in the case of the Carlitz module, these latter objects have similar properties to that of the ideal class groups and units of number fields (see for example [7). While Greenberg's Conjectures are "vertical" problems (one fixes a cyclotomic field and study the structures of the $p$-class groups along the cyclotomic $\mathbb{Z}_{p}$-extension), in this article we consider analogues of these problems for Taelman's class modules but in the "horizontal" case.

Let $\mathbb{F}_{q}$ be a finite field having $q$ elements and let $\theta$ be an indeterminate over $\mathbb{F}_{q}$. Let $\chi:\left(\frac{A}{f A}\right)^{\times} \rightarrow\left(K^{a c}\right)^{\times}$be a Dirichlet character of conductor $f$ (see section 4.1), where $A=\mathbb{F}_{q}[\theta]$, and $K^{a c}$ is a fixed algebraic closure of $K=\mathbb{F}_{q}(\theta)$. Let $L$ be the $f$ th cyclotomic function field (see 25, chapter 12). Let $C$ be the Carlitz module (see [13], chapter 3, and also section [2.6). Let's consider the isotypic component of Taelman's class module ([26, 27]) attached to the $f$ th cyclotomic function field :

$$
H_{\chi}:=e_{\chi}\left(\frac{L_{\infty}}{O_{L}+\exp _{C}\left(L_{\infty}\right)} \otimes_{\mathbb{F}_{q}} \mathbb{F}_{q}(\chi)\right)
$$

where $\exp _{C}$ is the Carlitz exponential map ([13], chapter 3), $O_{L}$ is the integral closure of $A$ in $L, \mathbb{F}_{q}(\chi) \subset K^{a c}$ is the finite extension of $\mathbb{F}_{q}$ obtained by adjoining to $\mathbb{F}_{q}$ the values of $\chi$, and $e_{\chi} \in \mathbb{F}_{q}(\chi)[\operatorname{Gal}(L / K)]$ is the usual idempotent associated to $\chi$ (note that $f$ is square free). Then L. Taelman proved that $H_{\chi}$ is a finite $A[\chi]:=$ $\mathbb{F}_{q}(\chi)[\theta]$-module (see for example [26]). In this article, we study the $A[\chi]$-module structure of $H_{\chi}$ when $\chi$ runs through the infinite family of Dirichlet characters of a given type (see 4.1).

Let $n \geq 1$ be an integer and let $t_{1}, \ldots, t_{n}$ be $n$ variables over $K_{\infty}:=\mathbb{F}_{q}\left(\left(\frac{1}{\theta}\right)\right)$. Let $\mathbb{T}_{n}\left(K_{\infty}\right)$ be the Tate algebra in the variables $t_{1}, \ldots, t_{n}$ with coefficients in $K_{\infty}$ (see [12], chapter 3). Let $\tau: \mathbb{T}_{n}\left(K_{\infty}\right) \rightarrow \mathbb{T}_{n}\left(K_{\infty}\right)$ be the continuous morphism of $\mathbb{F}_{q}\left[t_{1}, \ldots, t_{n}\right]$-algebras such that $\forall x \in K_{\infty}, \tau(x)=x^{q}$. Let $\phi: A \rightarrow A\left[t_{1}, \ldots, t_{n}\right]\{\tau\}$ be the morphism of $\mathbb{F}_{q}$-algebras such that:

$$
\phi_{\theta}=\left(t_{1}-\theta\right) \cdots\left(t_{n}-\theta\right) \tau+\theta .
$$


Let $\exp _{\phi}$ be the exponential function attached to $\phi$, i.e. $\exp _{\phi}$ is the unique element in $\mathbb{T}_{n}\left(K_{\infty}\right)\{\{\tau\}\}$ such that $\exp _{\phi} \equiv 1(\bmod \tau)$ and:

$$
\exp _{\phi} \theta=\phi_{\theta} \exp _{\phi} \text {. }
$$

Then inspired by Taelman's work ([26], 27, [28]), one can introduce a "generic class module" of level $n$ (see [5]):

$$
H_{n}:=\frac{\mathbb{T}_{n}\left(K_{\infty}\right)}{A\left[t_{1}, \ldots, t_{n}\right]+\exp _{\phi}\left(\mathbb{T}_{n}\left(K_{\infty}\right)\right)} .
$$

Observe that $H_{n}$ is an $A\left[t_{1}, \ldots, t_{n}\right]$-module via $\phi$, furthermore it is a finitely generated $\mathbb{F}_{q}\left[t_{1}, \ldots, t_{n}\right]$-module and a torsion $A\left[t_{1}, \ldots, t_{n}\right]$-module (we refer the reader to section 3 for more details). The terminology "generic class module" comes from the fact that the evaluations of elements of $\mathbb{T}_{n}\left(K_{\infty}\right)$ on elements of ${\overline{\mathbb{F}_{q}}}^{n}\left(\overline{\mathbb{F}_{q}}\right.$ being the algebraic closure of $\mathbb{F}_{q}$ in $K^{a c}$ ) induce surjective morphisms from $H_{n}$ to isotypic components of Taelman's class modules associated to Dirichlet characters of type $n$. These generic class modules can be viewed as discrete analogues of the Iwasawa modules discussed above, having in mind that the role of $\mathbb{Z}_{p}$ is played by $\mathbb{F}_{q}\left[t_{1}, \ldots, t_{n}\right]$, the role of $T$ is played by $\phi_{\theta}$. For example Mazur-Wiles Theorem has an analogue in this situation, let $n \geq q, n \equiv 1(\bmod q-1)$ (this is the analogue of the condition $\chi$ odd, $\chi \neq \omega_{p}$, for Iwasawa modules), then there exists $\mathbb{B}\left(t_{1}, \ldots, t_{n}\right) \in A\left[t_{1}, \ldots, t_{n}\right]$ which is a monic polynomial in $\theta$, such that the Fitting ideal of the $A\left[t_{1}, \ldots, t_{n}\right]$-module $H_{n}$ is generated by $\mathbb{B}\left(t_{1}, \ldots, t_{n}\right)$ and ([5], Theorem 7.7):

$$
(-1)^{\frac{n-1}{q-1}} \mathbb{B}\left(t_{1}, \ldots, t_{n}\right) \frac{\widetilde{\pi}}{\omega\left(t_{1}\right) \cdots \omega\left(t_{n}\right)}=L\left(t_{1}, \ldots, t_{n}\right),
$$

where $L\left(t_{1}, \ldots, t_{n}\right) \in \mathbb{T}_{n}\left(K_{\infty}\right)^{\times}$is the $L$-series attached to $\phi / A\left[t_{1}, \ldots, t_{n}\right]$ (see section 3), and $\omega(t)$ is the Anderson-Thakur special function (see [2], and also [5]). The reader can now easily guess what are the discrete analogues of Greenberg's Conjectures in our situation. We prove these discrete Greenberg's Conjectures in section 3.3 which was left as open questions in [5], more precisely:

Pseudo-cyclicity: let $n \geq 2, n \equiv 1(\bmod q-1)$, there exists an injective morphism of $A\left[t_{1}, \ldots, t_{n}\right]$-modules between a cyclic $A\left[t_{1}, \ldots, t_{n}\right]$-module and $H_{n}$ such that the cokernel of this morphism is a finitely generated and torsion $\mathbb{F}_{q}\left[t_{1}, \ldots, t_{n}\right]$-module (Theorem 3.17);

Pseudo-nullity: let $n \geq 2, n \not \equiv 1(\bmod q-1)$, then $H_{n}$ is a finitely generated and torsion $\mathbb{F}_{q}\left[t_{1}, \ldots, t_{n}\right]$-module (Theorem 3.10).

By [5], Theorem 3.10 and Theorem 3.17 imply that there exists $F\left(t_{1}, \ldots, t_{n}\right) \in$ $\mathbb{F}_{q}\left[t_{1}, \ldots, t_{n}\right] \backslash\{0\}$ (depending on the module structure of $H_{n}$ ) such that for every Dirichlet character $\chi$ of type $n$ verifying $F\left(\eta_{1}, \ldots, \eta_{n}\right) \neq 0$, then, if $n \equiv 1(\bmod q-$ $1), H_{\chi}$ is a cyclic $A[\chi]$-module and if $n \not \equiv 1(\bmod q-1)$ we have $H_{\chi}=\{0\}$.

The paper is organized as follows: in section 2, we introduce a natural submodule of finite index in the module of Taelman's units associated to a Drinfeld module : the module of Stark units; we prove several basic properties of this module and study its connection to $L$-series. In section 3, we show how the constructions of section 2 can also be made in the context of deformation of Drinfeld modules over Tate algebras; we then study in deep the arithmetic properties of Stark units attached to deformations of the Carlitz module and their connections to $L$-series leading to the proof of the discrete Greenberg Conjectures. In the last section, 
combining the ideas developed in section 2 and section [3, we prove a cyclicity result involving the "derivatives" of Dirichlet-Goss $L$-series.

The authors warmly thank David Goss for fruitful comments on an earlier version of this article.

\section{Stark Units}

In this section, we construct a natural submodule of the module of Taelman's units associated to a Drinfeld module defined over the ring of integers of a finite extension of $K$. The ideas developed in this section will be used in section 3 to prove the discrete Greenberg Conjectures.

\subsection{Preliminaries.}

Let $k$ be a field and let $\theta$ be an indeterminate over $k$. We set $R=k[\theta]$ and $\mathbb{K}=k\left(\left(\frac{1}{\theta}\right)\right)$. We endow $\mathbb{K}$ with the $\frac{1}{\theta}$-adic topology. An element $x \in \mathbb{K}^{\times}$is said to be monic if $x=\frac{1}{\theta^{m}}+\sum_{i>m} x_{i} \frac{1}{\theta^{i}}, m \in \mathbb{Z}, x_{i} \in k$.

Let $M$ be a finite dimensional $k$-vector space which is an $R$-module, then there exist $r_{1}, \ldots, r_{n}$ which are monic elements in $R$ such that we have an isomorphism of $R$-modules:

$$
M \simeq \prod_{j=1}^{n} \frac{R}{r_{j} R}
$$

We set:

$$
[M]_{R}=r_{1} \cdots r_{n}
$$

the monic generator of the Fitting ideal of $M$. Observe that:

$$
[M]_{R}=\left.\operatorname{det}_{k[X]}\left((1 \otimes X) \operatorname{Id}-\left.(\theta \otimes 1)\right|_{M \otimes_{k} k[X]}\right)\right|_{X=\theta} .
$$

Therefore, if $M_{1}, M, M_{2}$ are $R$-modules such that $M$ is a finite dimensional $k$-vector space, and suppose that we have a short exact sequence of $R$-modules:

$$
0 \rightarrow M_{1} \rightarrow M \rightarrow M_{2} \rightarrow 0,
$$

then:

$$
[M]_{R}=\left[M_{1}\right]_{R}\left[M_{2}\right]_{R}
$$

Let $V$ be a finite dimensional $\mathbb{K}$-vector space. We recall the following basic fact:

Lemma 2.1. Let $M$ be a sub $R$-module of $V$ such that $M$ is discrete in $V$. Let $W$ be the sub $\mathbb{K}$-vector space of $V$ generated by $M$. Then $M$ is a finitely generated and free $R$-module of rank $\operatorname{dim}_{\mathbb{K}} W$.

Proof. Set $n=\operatorname{dim}_{\mathbb{K}} W$. Choose $\|\cdot\|$ a norm of $\mathbb{K}$-vector space on $W$. Since $W$ is closed in $V$, we deduce that $M$ is discrete in $W$. Thus there exists $m \in M \backslash\{0\}$ such that $\|m\|$ is minimal, so that $R m=M \cap \mathbb{K} m$. In particular, when $\operatorname{dim}_{\mathbb{K}} W=1$, then $M=R m$ is free of rank 1. Let $M^{\prime}$ be the image of $M$ in $W / \mathbb{K} m$. By induction, $M^{\prime}$ is free of rank $n-1$. Since we have a short exact sequence of torsion-free $R$-modules

$$
0 \rightarrow R m \rightarrow M \rightarrow M^{\prime} \rightarrow 0
$$

we deduce that $M$ is a free $R$-module of rank $n$. 
An $R$-lattice in $V, M$, is a sub $R$-module of $V$ such that $M$ is discrete in $V$ and $M$ contains a $\mathbb{K}$-basis of $V$. In particular, by the above Lemma, $M$ is a free $R$-module of rank $\operatorname{dim}_{\mathbb{K}} V$.

Let $M_{1}$ and $M_{2}$ be two $R$-lattices in $V$. Let $n=\operatorname{dim}_{\mathbb{K}} V$. Following [27, we will define $\left[M_{1}: M_{2}\right]_{R} \in \mathbb{K}^{\times}$. Select $e_{1}, \ldots, e_{n}$ in $M_{1}$ and $f_{1}, \ldots, f_{n} \in M_{2}$ such that:

$$
M_{1}=\oplus_{j=1}^{n} R e_{j} \text { and } M_{2}=\oplus_{j=1}^{n} R f_{j}
$$

Then, observe that:

$$
M_{1}=\oplus_{j=1}^{n} \mathbb{K} e_{j}=\oplus_{j=1}^{n} \mathbb{K} f_{j} .
$$

Let $f: V \rightarrow V$ be the $\mathbb{K}$-linear map such that $f\left(e_{i}\right)=f_{i}, i=1, \ldots, n$. Let $B$ be the matrix of $f$ in the basis $\left(e_{i}\right)_{i=1, \ldots, n}$. then $B \in G L_{n}(\mathbb{K})$ and $(\operatorname{det} B) k^{\times}$does not depend on the choices of $\left(e_{i}\right)_{i=1, \ldots, n}$ and $\left(f_{i}\right)_{i=1, \ldots . n}$. Let $x \in \mathbb{K}^{\times}$be the monic element such that $(\operatorname{det} B) k^{\times}=x k^{\times}$, then we set:

$$
\left[M_{1}: M_{2}\right]_{R}=x .
$$

Observe that $\left[M_{1}: M_{2}\right]_{R}$ is well-defined.

If $M_{2} \subset M_{1}$ then $\frac{M_{1}}{M_{2}}$ is a finite dimensional $k$-vector space. Since $R$ is a principal ideal domain, there exists an $R$-basis $\left(e_{1}, \ldots, e_{n}\right)$ of $M_{1}, r_{1}, \ldots, r_{t}, t$ monic elements in $R, t \leq n$, such that if we set $f_{i}=r_{i} e_{i}, i=1, \ldots, t, f_{i}=e_{i}, t+1 \leq i \leq n$, then $\left(f_{1}, \ldots, f_{n}\right)$ is an $R$-basis of $M_{2}$. Thus, we have:

$$
\left[M_{1}: M_{2}\right]_{R}=\left[\frac{M_{1}}{M_{2}}\right]_{R}
$$

If $M_{1}, M_{2}, M_{3}$ are three $R$-lattices in $V$, then:

$$
\left[M_{1}: M_{3}\right]_{R}=\left[M_{1}: M_{2}\right]_{R}\left[M_{2}: M_{3}\right]_{R} .
$$

Let $M_{1}, M_{2}$ be two $R$-lattices in $V$. Let $W$ be a sub $\mathbb{K}$-vector space of $V$ and let $N_{1}, N_{2}$ be two $R$-lattices in $W$ such that:

$$
M_{i} \cap W=N_{i}, i=1,2 .
$$

Then $\frac{M_{1}}{N_{1}}$ and $\frac{M_{2}}{N_{2}}$ are two $R$-lattices in $\frac{V}{W}$ and we have:

$$
\left[\frac{M_{1}}{N_{1}}: \frac{M_{2}}{N_{2}}\right]_{R}=\frac{\left[M_{1}: M_{2}\right]_{R}}{\left[N_{1}: N_{2}\right]_{R}} .
$$

Let $G$ be a finite abelian group, we assume that $|G|$ is prime to the characteristic of $k$. Let $\bar{k}$ be an algebraic closure of $k$. We set $\widehat{G}=\operatorname{Hom}\left(G, \bar{k}^{\times}\right)$. For $\chi \in \widehat{G}$, let $k(\chi)=k(\chi(g), g \in G) \subset \bar{k}, R(\chi)=k(\chi)[\theta]$, and $\mathbb{K}(\chi)=k(\chi)\left(\left(\frac{1}{\theta}\right)\right)$.

For $\chi \in \widehat{G}$, we set:

$$
e_{\chi}=\frac{1}{|G|} \sum_{g \in G} \chi(g) g^{-1} \in k(\chi)[G]
$$

We also set:

$$
[\chi]=\{\psi \in \widehat{G}, \psi=\sigma \circ \chi \text { for some } \sigma \in \operatorname{Gal}(k(\chi) / k)\},
$$

and:

$$
e_{[\chi]}=\sum_{\psi \in[\chi]} e_{\psi} \in k[G]
$$


Let $M$ be a $k[G]$-module. Then, for $\chi \in \widehat{G}$, we set:

$$
M(\chi)=e_{\chi}\left(M \otimes_{k} k(\chi)\right) .
$$

We have :

$$
\oplus_{\psi \in[\chi]} M(\psi)=\left(e_{[\chi]} M\right) \otimes_{k} k(\chi) .
$$

Let $f \in R[G] \cap \operatorname{Frac}(R)[G]^{\times}$and let $\rho_{f}: R[G] \rightarrow R[G]$ be the morphism of $R$-modules given by the multiplication by $f$. Let $\operatorname{det}_{R} \rho_{f} \in R \cap \operatorname{Frac}(R)^{\times}$be the determinant of the matrix of $\rho_{f}$ with respect to the $R$-basis given by the elements of $G$, then there exists a unique monic element $\operatorname{det}_{R} f \in R$ such that:

$$
\left(\operatorname{det}_{R} f\right) k^{\times}=\left(\operatorname{det}_{R} \rho_{f}\right) k^{\times} .
$$

Observe that $\operatorname{det}_{R} f$ is well-defined. For $\chi \in \widehat{G}$, we define $f(\chi) \in R(\chi)$ by the equality:

$$
e_{\chi} f=f(\chi) e_{\chi}
$$

We get:

$$
\left(\operatorname{det}_{R} f\right) k^{\times}=\left(\prod_{\chi \in \widehat{G}} f(\chi)\right) k^{\times}
$$

Let $M$ be a finite dimensional $k$-vector space which is an $R[G]$-module. Let $L / k$ be a finite extension containing $k(\chi)$, then $\left[e_{\chi}\left(M \otimes_{k} L\right)\right]_{L[\theta]}$ does not depend on $L$. Observe that:

$$
\forall \sigma \in \operatorname{Gal}(k(\chi) / k), \sigma\left([M(\chi)]_{R(\chi)}\right)=[M(\sigma \circ \chi)]_{R(\chi)} .
$$

We set:

$$
[M]_{R[G]}=\sum_{\chi \in \widehat{G}}[M(\chi)]_{R(\chi)} e_{\chi} \in R[G] \cap \operatorname{Frac}(R)[G]^{\times} .
$$

Then:

$$
\operatorname{det}_{R}[M]_{R[G]}=\prod_{\chi \in \widehat{G}}[M(\chi)]_{R(\chi)}=[M]_{R} .
$$

Finally, observe that if $M_{1}, M, M_{2}$ are finite dimensional $k$-vector spaces that are also $R[G]$-modules and such that we have an exact sequence of $R[G]$-modules:

$$
O \rightarrow M_{1} \rightarrow M \rightarrow M_{2} \rightarrow 0,
$$

then:

$$
[M]_{R[G]}=\left[M_{1}\right]_{R[G]}\left[M_{2}\right]_{R[G]} .
$$

Let $V$ be a finite dimensional $\mathbb{K}$-vector space which is a free $\mathbb{K}[G]$-module. An $R[G]$-lattice in $V$, is an $R$-lattice in $V, M$, such that $M$ is an $R[G]$-module. Observe that $M$ is a free $R[G]$-module: for $\chi \in \widehat{G}, e_{[\chi]} M$ is a finitely generated $e_{[\chi]} R[G]$ module without torsion and thus a free $e_{[\chi]} R[G]$-module $\left(e_{[\chi]} R[G]\right.$ is a principal ideal domain). Let $\chi \in \widehat{G}$, then $M(\chi)$ is an $R(\chi)$-lattice in the finite dimensional $\mathbb{K}(\chi)$-vector space $V(\chi)$.

Let $M_{1}, M_{2}$ be two $R[G]$-lattices in $V$. Observe that:

$$
\forall \sigma \in \operatorname{Gal}(k(\chi) / k), \sigma\left(\left[M(\chi): M_{2}(\chi)\right]_{R(\chi)}\right)=\left[M_{1}(\sigma \circ \chi): M_{2}(\sigma \circ \chi)\right]_{R(\chi)} .
$$


We set:

$$
\left[M_{1}: M_{2}\right]_{R[G]}=\sum_{\chi \in \widehat{G}}\left[M_{1}(\chi): M_{2}(\chi)\right]_{R(\chi)} e_{\chi} \in \mathbb{K}[G]^{\times}
$$

We have:

$$
\left[M_{1}: M_{2}\right]_{R}=\prod_{\chi \in \widehat{G}}\left[M_{1}(\chi): M_{2}(\chi)\right]_{R(\chi)} .
$$

Furthermore, if $M_{2} \subset M_{1}$, we have:

$$
\left[M_{1}: M_{2}\right]_{R[G]}=\left[\frac{M_{1}}{M_{2}}\right]_{R[G]} .
$$

Finally if $M_{1}, M_{2}, M_{3}$ are three $R[G]$-lattices in $V$, then:

$$
\left[M_{1}: M_{3}\right]_{R[G]}=\left[M_{1}: M_{2}\right]_{R[G]}\left[M_{2}: M_{3}\right]_{R[G]} .
$$

\section{2. $\mathbb{F}_{q}(z)[\theta]$-Drinfeld modules.}

Let $\mathbb{F}_{q}$ be a finite field having $q$ elements and let $p$ be the characteristic of $\mathbb{F}_{q}$. Let $\theta$ be an indeterminate over $\mathbb{F}_{q}$ and let $A=\mathbb{F}_{q}[\theta]$. Let $A_{+}$be the set of monic polynomials in $A$. For $n \geq 0$, we denote by $A_{+, n}$ the set of elements of $A_{+}$of degree $n$. Let $K=\mathbb{F}_{q}(\theta)$ and $K_{\infty}=\mathbb{F}_{q}\left(\left(\frac{1}{\theta}\right)\right)$. Let $\mathbb{C}_{\infty}$ be the completion of a fixed algebraic closure of $K_{\infty}$ and let $v_{\infty}: \mathbb{C}_{\infty} \rightarrow \mathbb{Q} \cup\{+\infty\}$ be the valuation on $\mathbb{C}_{\infty}$ normalized such that $v_{\infty}(\theta)=-1$.

Let $z$ be an indeterminate over $\mathbb{C}_{\infty}$. We still denote by $v_{\infty}$ the $\infty$-adic Gauss valuation on $\mathbb{C}_{\infty}(z)$, i.e. if $f \in \mathbb{C}_{\infty}[z], f=\sum_{k=0}^{n} f_{n} z^{n}, f_{n} \in \mathbb{C}_{\infty}, v_{\infty}(f)=$ $\operatorname{Inf}\left\{v_{\infty}\left(f_{k}\right), k \in\{0, \ldots, n\}\right\}$. Let $F$ be a subfield of $\mathbb{C}_{\infty}$ containing $K_{\infty}$ and complete for $v_{\infty}$, we denote by $\mathbb{T}_{z}(F)$ the completion of $F[z]$ for $v_{\infty}$ and $\widetilde{F}$ the completion of $F(z)$ for $v_{\infty}$. Then the $\mathbb{F}_{q}(z)$-vector space generated by $\mathbb{T}_{z}(F)$ is dense in $\widetilde{F}$.

Let $L / K$ be a finite extension and let $L_{\infty}=L \otimes_{K} K_{\infty}$. Let $O_{L}$ be the integral closure of $A$ in $L$. Let $S_{\infty}(L)$ be the set of places of $L$ above $\infty$. For each $v \in S_{\infty}(L)$, let $\iota_{v}: L \rightarrow \mathbb{C}_{\infty}$ be the morphism of $K$-algebras corresponding to $v$, we denote by $L_{v}$ the field generated over $K_{\infty}$ by $\iota_{v}(L)$. Let's set:

$$
\widetilde{L_{\infty}}=L \otimes_{K} \widetilde{K_{\infty}} \text {. }
$$

Observe that we have an isomorphism of $\widetilde{K_{\infty}}$-algebras:

$$
\widetilde{L_{\infty}} \simeq \prod_{v \in S_{\infty}(L)} \widetilde{L_{v}}
$$

Let $\tau: \widetilde{L_{\infty}} \rightarrow \widetilde{L_{\infty}}$ be the continuous morphism of $\mathbb{F}_{q}(z)$-algebras such that $\forall x \in$ $L, \tau(x)=x^{q}$. Let $\widetilde{O_{L}}$ be the $\mathbb{F}_{q}(z)$-vector space generated by $O_{L}$ in $\widetilde{L_{\infty}}$, and set $\widetilde{A}=\mathbb{F}_{q}(z)[\theta]$.

A Drinfeld $\widetilde{A}$-module defined over $\widetilde{O_{L}}, \varphi$, is a morphism of $\mathbb{F}_{q}(z)$-algebras $\varphi$ : $\widetilde{A} \rightarrow \widetilde{L_{\infty}}\{\tau\}$ such that $\varphi_{\theta} \equiv \theta(\bmod \tau)$. We denote by $\varphi^{0}$ the trivial $\widetilde{A}$-Drinfeld module, i.e. $\varphi_{\theta}^{0}=\theta$.

Let $\varphi$ be an $\widetilde{A}$-Drinfeld module defined over $\widetilde{O_{L}}$, then there exists a unique element $\exp _{\varphi} \in L(z)\{\{\tau\}\}$ such that ([9], Proposition 2.3):

$$
\begin{gathered}
\exp _{\varphi} \equiv 1 \quad(\bmod \tau) \\
\exp _{\varphi} \theta=\varphi_{\theta} \exp _{\varphi} .
\end{gathered}
$$


Let's set:

$$
H\left(\varphi / \widetilde{O_{L}}\right)=\frac{\widetilde{L_{\infty}}}{\widetilde{O_{L}}+\exp _{\varphi}\left(\widetilde{L_{\infty}}\right)} .
$$

Then, by 9 , Proposition 2.6, $H\left(\varphi / \widetilde{O_{L}}\right)$ is a finite dimensional $\mathbb{F}_{q}(z)$-vector space and an $\widetilde{A}$-module via $\varphi$. Furthermore, by [9, Proposition 2.6, $\exp _{\varphi}^{-1}\left(\widetilde{O_{L}}\right)$ is an $\widetilde{A}$-lattice in $\widetilde{L_{\infty}}$.

Let $\mathfrak{P}$ be a non zero prime ideal of $O_{L}$ and let us denote by $\varphi\left(\frac{\widetilde{O_{L}}}{\mathfrak{P} O_{L}}\right)$ the $\mathbb{F}_{q}(z)$ vector space $\frac{\widetilde{O_{L}}}{\mathfrak{O _ { L }}}$ viewed as an $\widetilde{A}$-module via $\varphi$. Then, by [9], Theorem 2.7 , the following infinite product converges in ${\widetilde{K_{\infty}}}^{\times}$:

$$
\mathcal{L}\left(\varphi / \widetilde{O_{L}}\right):=\prod_{\mathfrak{P} \text { prime ideal of } O_{L}, \mathfrak{P} \neq(0)} \frac{\left[\frac{\widetilde{O_{L}}}{\mathfrak{P} O_{L}}\right]_{\widetilde{A}}}{\left[\varphi\left(\frac{\widetilde{O_{L}}}{\mathfrak{P} O_{L}}\right)\right]_{\widetilde{A}}} \in{\widetilde{K_{\infty}}}^{\times} .
$$

Furthermore, we have ([9], Theorem 2.7):

$$
\mathcal{L}\left(\varphi / \widetilde{O_{L}}\right)=\left[\widetilde{O_{L}}: \exp _{\varphi}^{-1}\left(\widetilde{O_{L}}\right)\right]_{\widetilde{A}}\left[H\left(\varphi / \widetilde{O_{L}}\right)\right]_{\widetilde{A}} .
$$

Observe that $\mathcal{L}\left(\varphi^{0} / \widetilde{O_{L}}\right)=1$, $\exp _{\varphi^{0}}=1, \exp _{\varphi^{0}}^{-1}\left(\widetilde{O_{L}}\right)=\widetilde{O_{L}}, H\left(\varphi^{0} / \widetilde{O_{L}}\right)=\{0\}$.

\subsection{Integrality results.}

Let $\varphi$ be an $\widetilde{A}$-Drinfeld module defined over $\widetilde{O_{L}}$ such that $\varphi_{\theta} \in O_{L}[z]\{\tau\}$. Denote by $\mathbb{T}_{z}\left(L_{\infty}\right)$ the closure of $L_{\infty}[z]$ in $\widetilde{L_{\infty}}$. Then, we have an isomorphism of $\mathbb{T}_{z}\left(K_{\infty}\right)$ algebras:

$$
\mathbb{T}_{z}\left(L_{\infty}\right) \simeq \prod_{v \in S_{\infty}(L)} \mathbb{T}_{z}\left(L_{v}\right)
$$

Observe that $\tau \mid \mathbb{T}_{z}\left(L_{\infty}\right): \mathbb{T}_{z}\left(L_{\infty}\right) \rightarrow \mathbb{T}_{z}\left(L_{\infty}\right)$ is a continuous morphism of $\mathbb{F}_{q}[z]$ algebras. Since $\exp _{\varphi} \theta=\varphi_{\theta} \exp _{\varphi}$ and $\exp _{\varphi} \equiv 1(\bmod \tau)$, we deduce:

$$
\exp _{\varphi} \in \mathbb{T}_{z}\left(L_{\infty}\right)\{\{\tau\}\}
$$

Let's set:

$$
H\left(\varphi / O_{L}[z]\right)=\frac{\mathbb{T}_{z}\left(L_{\infty}\right)}{O_{L}[z]+\exp _{\varphi}\left(\mathbb{T}_{z}\left(L_{\infty}\right)\right)} .
$$

Let $n=\operatorname{dim}_{K} L$ and let $e_{1}, \ldots, e_{n} \in O_{L}$ such that $O_{L}=\oplus_{i=1}^{n} A e_{i}$. Then:

$$
\mathbb{T}_{z}\left(L_{\infty}\right)=\oplus_{i=1}^{n} \mathbb{T}_{z}\left(K_{\infty}\right) e_{i}
$$

Let $M=\left\{x \in \mathbb{T}_{z}\left(K_{\infty}\right), v_{\infty}(x) \geq 1\right\}$, then:

$$
\mathbb{T}_{z}\left(L_{\infty}\right)=O_{L}[z] \oplus \oplus_{i=1}^{n} M e_{i} .
$$

Since, by [9], Proposition 2.3, there exists an integer $N \geq 0$ such that $\exp _{\varphi}$ induces an isomorphism of $\mathbb{F}_{q}[z]$-module on $\oplus_{i=1}^{n} \frac{1}{\theta^{\mathbb{N}}} M e_{i}$, we deduce that $H\left(\varphi / O_{L}[z]\right)$ is a finitely generated $\mathbb{F}_{q}[z]$-module.

\section{Lemma 2.2.}

$$
\mathcal{L}\left(\varphi / \widetilde{O_{L}}\right) \in \mathbb{T}_{z}\left(K_{\infty}\right)^{\times}
$$


Proof. Let $\mathfrak{P}$ be a non zero prime ideal in $O_{L}$. Then we have an isomorphism of $\widetilde{A}$-modules:

$$
\frac{\widetilde{O_{L}}}{\mathfrak{P} \widetilde{O_{L}}} \simeq \frac{O_{L}}{\mathfrak{P}}[z] \otimes_{\mathbb{F}_{q}[z]} \mathbb{F}_{q}(z)
$$

Since $\frac{O_{L}}{\mathfrak{P}}[z]$ is a free $\mathbb{F}_{q}[z]$-module, we deduce that:

$$
\left[\varphi\left(\frac{\widetilde{O_{L}}}{\mathfrak{P} \widetilde{O_{L}}}\right)\right]_{\widetilde{A}}=\left.\operatorname{det}_{\mathbb{F}_{q}[z, X]}\left(X \operatorname{Id}-\left.\varphi_{\theta}\right|_{\frac{O_{L}}{\mathfrak{P}}[z]}\right)\right|_{X=\theta} \in A[z] .
$$

But :

$$
\operatorname{deg}_{\theta}\left[\varphi\left(\frac{\widetilde{O_{L}}}{\mathfrak{P} \widetilde{O_{L}}}\right)\right]_{\widetilde{A}}=\operatorname{dim}_{\mathbb{F}_{q}} \frac{\widetilde{O_{L}}}{\mathfrak{P}}=\operatorname{deg}_{\theta}\left[\frac{\widetilde{O_{L}}}{\mathfrak{P}}\right]_{\widetilde{A}}
$$

Therefore $\left[\varphi\left(\frac{\widetilde{O_{L}}}{\mathfrak{P O} \widetilde{O}_{L}}\right)\right]_{\widetilde{A}}$ is a monic polynomial in $\theta$ in $A[z]$ having the same degree as $\left[\frac{\widetilde{O_{L}}}{\mathfrak{P}^{\mathfrak{A}}}\right]_{\widetilde{A}}$ which is a monic polynomial in $A$. We get:

$$
\frac{\left[\frac{\widetilde{O_{L}}}{\mathfrak{P} O_{L}}\right]_{\widetilde{A}}}{\left[\varphi\left(\frac{\widetilde{O_{L}}}{\mathfrak{P} \widetilde{O}_{L}}\right)\right]_{\widetilde{A}}} \in 1+\frac{1}{\theta} \mathbb{F}_{q}[z]\left[\left[\frac{1}{\theta}\right]\right] .
$$

Since, by [9], Theorem 2.7, the infinite product $\prod_{\mathfrak{P} \text { prime ideal of } O_{L}, \mathfrak{P} \neq(0)} \frac{\left[\frac{\widetilde{O}_{L}}{\mathfrak{P O} O_{L}}\right]}{\left[\varphi\left(\frac{\overline{O_{L}}}{\mathfrak{P} O_{L}}\right)\right]_{\tilde{A}}}$ converges in $\widetilde{K_{\infty}}$, we deduce that:

$$
\mathcal{L}\left(\varphi / \widetilde{O_{L}}\right) \in 1+\frac{1}{\theta} \mathbb{F}_{q}[z]\left[\left[\frac{1}{\theta}\right]\right]
$$

\section{Proposition 2.3.}

1) Let $U\left(\varphi / O_{L}[z]\right)=\left\{x \in \mathbb{T}_{z}\left(L_{\infty}\right), \exp _{\varphi}(x) \in O_{L}[z]\right\}$. Then $\exp _{\varphi}^{-1}\left(\widetilde{O_{L}}\right)$ is the $\mathbb{F}_{q}(z)$-vector space generated by $U\left(\varphi / O_{L}[z]\right)$.

2) $U\left(\varphi / O_{L}[z]\right)$ is a finitely generated $A[z]$-module.

Proof.

1) The proof is similar to that of [5, Proposition 5.4. For simplicity let's denote by $V$ the $\mathbb{F}_{q}(z)$-vector space generated by $U\left(\varphi / O_{L}[z]\right)$. Then clearly $V \subset \exp _{\varphi}^{-1}\left(\widetilde{O_{L}}\right)$. Let $N$ be a suitable neighborhood of zero in $\widetilde{L_{\infty}} \operatorname{such}$ that $\exp _{\varphi}: N \rightarrow N$ is an isomorphism of $\mathbb{F}_{q}(z)$-vector spaces. Let $W$ be the $\mathbb{F}_{q}(z)$-vector space generated by $\mathbb{T}_{z}\left(L_{\infty}\right)$, then:

$$
\widetilde{L_{\infty}}=W+N .
$$

Let $f \in \exp _{\varphi}^{-1}\left(\widetilde{O_{L}}\right)$, then we can write:

$$
f=g+h, g \in W, h \in N \text {. }
$$

Then:

$$
\exp _{\varphi}(h)=\exp _{\varphi}(g)-\exp _{\varphi}(f) \in W \cap N .
$$

Therefore $h \in W$. This implies that $f \in V$. 
2) Since $\exp _{\varphi}^{-1}\left(\widetilde{O_{L}}\right)$ is discrete in $\widetilde{L_{\infty}}$, we deduce that $U\left(\varphi / O_{L}[z]\right)$ is discrete in $\mathbb{T}_{z}\left(L_{\infty}\right)$. Furthermore, the $\widetilde{K_{\infty}}$-vector space generated by $\exp _{\varphi}^{-1}\left(\widetilde{O_{L}}\right)$ is $\widetilde{L_{\infty}}$, thus we can select $f_{1}, \ldots, f_{n} \in U\left(\varphi / O_{L}[z]\right)$ such that:

$$
\begin{gathered}
\exp _{\varphi}^{-1}\left(\widetilde{O_{L}}\right)=\oplus_{i=1}^{n} \widetilde{A} f_{i}, \\
\widetilde{L_{\infty}}=\oplus_{i=1}^{n} \widetilde{K_{\infty}} f_{i} .
\end{gathered}
$$

Set $N=\oplus_{i=1}^{n} A[z] f_{i}$. Let $V$ be the $\mathbb{T}_{z}\left(K_{\infty}\right)$-module generated by $N$, then $V=$ $\oplus_{i=1}^{n} \mathbb{T}_{z}\left(K_{\infty}\right) f_{i}$. Let $W$ be the $\mathbb{T}_{z}\left(K_{\infty}\right)$-module generated by $U\left(\varphi / O_{L}[z]\right)$. Then : $V \subset W \subset \mathbb{T}_{z}\left(L_{\infty}\right)$. In particular, $W$ is a free $\mathbb{T}_{z}\left(K_{\infty}\right)$-module of rank $n=[L: K]$. Observe that, because of 1$), U\left(\varphi / O_{L}[z]\right) \subset \mathbb{F}_{q}(z) N$, that is, if $x \in U\left(\varphi / O_{L}[z]\right)$, then there exists $\delta \in \mathbb{F}_{q}[z] \backslash\{0\}$ such that $\delta x \in N$. This implies that there exists $\delta \in \mathbb{F}_{q}[z] \backslash\{0\}$ such that :

$$
\delta W \subset V .
$$

Thus:

$$
\delta U\left(\varphi / O_{L}[z]\right) \subset V \cap \mathbb{F}_{q}(z) N=N .
$$

This implies that $U\left(\varphi / O_{L}[z]\right)$ is a finitely generated $A[z]$-module.

\subsection{The canonical $z$-deformation of a Drinfeld module.}

Let $\phi: A \rightarrow O_{L}\{\tau\}$ be a Drinfeld $A$-module, i.e. it is a morphism of $\mathbb{F}_{q}$-algebras such that:

$$
\phi_{\theta} \equiv \theta \quad(\bmod \tau) .
$$

Let $\exp _{\phi}$ be the unique element in $L\{\{\tau\}\}$ such that $\exp _{\phi} \equiv 1(\bmod \tau)$ and:

$$
\exp _{\phi} \theta=\phi_{\theta} \exp _{\phi} \text {. }
$$

Following Taelman ([26]), let's set:

$$
H\left(\phi / O_{L}\right)=\frac{L_{\infty}}{O_{L}+\exp _{\phi}\left(L_{\infty}\right)} .
$$

Then $H\left(\phi / O_{L}\right)$ is a finite $A$-module (via $\left.\phi\right)$. Let's also set:

$$
U\left(\phi / O_{L}\right)=\left\{x \in L_{\infty}, \exp _{\phi}(x) \in O_{L}\right\} .
$$

Then $U\left(\phi / O_{L}\right)$ is an $A$-lattice in $L_{\infty}$ and we have (see [27]):

$$
\mathcal{L}\left(\phi / O_{L}\right)=\left[O_{L}: U\left(\phi / O_{L}\right)\right]_{A}\left[H\left(\phi / O_{L}\right)\right]_{A},
$$

where:

$$
\mathcal{L}\left(\phi / O_{L}\right)=\prod_{\mathfrak{P} \text { prime ideal of } O_{L}, \mathfrak{P} \neq(0)} \frac{\left[\frac{O_{L}}{\mathfrak{P}}\right]_{A}}{\left[\phi\left(\frac{O_{L}}{\mathfrak{P}}\right)\right]_{A}} \in K_{\infty}^{\times} .
$$

Write:

$$
\phi_{\theta}=\sum_{j=0}^{r} \alpha_{j} \tau^{j}, \alpha_{j} \in O_{L}, j=0, \ldots, r, \alpha_{0}=\theta .
$$

The canonical $z$-deformation of $\phi, \widetilde{\phi}$, is the $\widetilde{A}$-module defined over $\widetilde{O_{L}}$ given by:

$$
\widetilde{\phi}_{\theta}=\sum_{j=0}^{r} z^{j} \alpha_{j} \tau^{j} \in O_{L}[z]\{\tau\} .
$$

Then, one can easily verify that:

$$
\exp _{\tilde{\phi}}=\sum_{j \geq 0} e_{j} z^{j} \tau^{j}
$$


where $\exp _{\phi}=\sum_{j \geq 0} e_{j} \tau^{j}, e_{j} \in L, j \geq 0$.

Proposition 2.4. $H\left(\widetilde{\phi} / O_{L}[z]\right)$ is a finitely generated and torsion $\mathbb{F}_{q}[z]$-module. In particular:

$$
H\left(\widetilde{\phi} / \widetilde{O_{L}}\right)=\{0\} .
$$

Proof. By section $2.3, H\left(\widetilde{\phi} / O_{L}[z]\right)$ is a finitely generated $\mathbb{F}_{q}[z]$-module. Observe that, since $\exp _{\tilde{\phi}} \equiv 1 \bmod z$ :

$$
\mathbb{T}_{z}\left(L_{\infty}\right)=z \mathbb{T}_{z}\left(L_{\infty}\right)+\exp _{\widetilde{\phi}}\left(\mathbb{T}_{z}\left(L_{\infty}\right)\right) .
$$

Therefore, the multiplication by $z$ on $H\left(\widetilde{\phi} / O_{L}[z]\right)$ gives rise to an exact sequnece of finitely generated $\mathbb{F}_{q}[z]$-modules:

$$
0 \rightarrow H\left(\widetilde{\phi} / O_{L}[z]\right)[z] \rightarrow H\left(\widetilde{\phi} / O_{L}[z]\right) \rightarrow H\left(\widetilde{\phi} / O_{L}[z]\right) \rightarrow 0,
$$

where

$$
H\left(\widetilde{\phi} / O_{L}[z]\right)[z]=\left\{x \in H\left(\widetilde{\phi} / O_{L}[z]\right), z x=0\right\} .
$$

This implies that $H\left(\widetilde{\phi} / O_{L}[z]\right)[z]=\{0\}$ and that $H\left(\widetilde{\phi} / O_{L}[z]\right)$ is a torsion $\mathbb{F}_{q}[z]$ module. Since the $\mathbb{F}_{q}(z)$-module generated by $\mathbb{T}_{z}\left(L_{\infty}\right)$ is dense in $\widetilde{L_{\infty}}$, we deduce that the inclusion $\mathbb{T}_{z}\left(L_{\infty}\right) \subset \widetilde{L_{\infty}}$ induces an isomorphism of $\widetilde{A}$-modules:

$$
H\left(\widetilde{\phi} / O_{L}[z]\right)[z] \otimes_{\mathbb{F}_{q}[z]} \mathbb{F}_{q}(z) \simeq H\left(\widetilde{\phi} / \widetilde{O_{L}}\right) .
$$

This implies the second assertion of the Lemma.

Remark 2.5. Let $\phi$ be a Drinfeld $A$-module defined over $O_{L}$. Let $\mathfrak{P}$ be a non zero prime ideal of $O_{L}$. If $\phi_{\theta} \equiv \theta(\bmod \mathfrak{P})$ then:

$$
\left[\phi\left(\frac{O_{L}}{\mathfrak{P}}\right)\right]_{A}=\left[\frac{O_{L}}{\mathfrak{P}}\right]_{A}=N_{L / K}(\mathfrak{P}),
$$

where for $I \neq\{0\}$ an ideal of $O_{L}$ we set $N_{L / K}(I)=\left[\frac{O_{L}}{I}\right]_{A}$. We also have:

$$
\left[\widetilde{\phi}\left(\frac{\widetilde{O_{L}}}{\mathfrak{P} \widetilde{O_{L}}}\right)\right]_{\widetilde{A}}=N_{L / K}(\mathfrak{P})
$$

In this case, we set:

$$
f_{\mathfrak{P}}(z)=0 .
$$

Thus we assume that $\phi_{\theta} \not \equiv \theta(\bmod \mathfrak{P})$. Let $\bar{\phi}: A \rightarrow \frac{O_{L}}{\mathfrak{P}}\{\tau\}$ be the reduction modulo $\mathfrak{P}$ of $\phi$. Set $\mathfrak{L}=\frac{O_{L}}{\mathfrak{P}}$. Let $P$ be the monic irreducible element in $A$ such that $\mathfrak{P} \cap A=P A$. Then:

$$
N_{L / K}(\mathfrak{P})=P^{m},
$$

where $\operatorname{dim}_{\mathbb{F}_{q}} \mathfrak{L}=m \operatorname{deg}_{\theta}$ P. We have: $\frac{\widetilde{O_{L}}}{\mathfrak{P} \widetilde{O_{L}}}=\mathfrak{L}(z)$. Let's observe that:

$$
g_{\mathfrak{P}}(z):=[\widetilde{\phi}(\mathfrak{L}(z))]_{\widetilde{A}} \in A[z] .
$$

Furthermore:

$$
\begin{gathered}
g_{\mathfrak{P}}(0)=P^{m}, \\
g_{\mathfrak{P}}(1)=[\phi(\mathfrak{L})]_{A} .
\end{gathered}
$$


Let's make a simple observation. Let $M$ be a finitely generated and free Amodule. Let $u: M \rightarrow M$ be an injective morphism of A-modules. Then:

$$
\left(\operatorname{det}_{A} u\right) A=\left[\frac{M}{u(M)}\right]_{A} A .
$$

Let's view $\mathfrak{L}\{\tau\}$ as a left $A$-module via $\bar{\phi}$. Then it is a finitely generated and free $A$-module. We have an isomorphism of A-modules:

$$
\frac{\mathfrak{L}\{\tau\}}{\mathfrak{L}\{\tau\}(\tau-1)} \simeq \phi(\mathfrak{L}) .
$$

Let $\rho: \mathfrak{L}\{\tau\} \rightarrow \mathfrak{L}\{\tau\}$ be the morphism of A-modules given by :

$$
\rho(x)=x \tau \text {. }
$$

Then:

$$
\left(\operatorname{det}_{A}(\operatorname{Id}-\rho)\right) A=[\phi(\mathfrak{L})]_{A} A .
$$

Let $X$ be a variable, and set:

$$
F(X)=\operatorname{det}_{A[X]}\left((1 \otimes X) \operatorname{Id}-\left.\rho \otimes 1\right|_{\mathfrak{L}\{\tau\} \otimes_{\mathbb{F}_{q}} \mathbb{F}_{q}[X]}\right) \in A[X] .
$$

Since $\frac{\mathfrak{L}\{\tau\}}{\mathfrak{L}\{\tau\} \tau}$ is isomorphic as an A-module to $\mathfrak{L}$, we have:

$$
F(0) A=P^{m} A \text {. }
$$

Now, observe that we have an isomorphism of A-modules:

$$
\frac{\mathfrak{L}[z]\{\tau\}}{\mathfrak{L}[z]\{\tau\}(\tau-z)} \simeq \widetilde{\phi}(\mathfrak{L}[z])
$$

Thus:

$$
g_{\mathfrak{P}}(z) A[z]=F(z) A[z] .
$$

Let's set $f_{\mathfrak{P}}(z)=P^{m}-g_{\mathfrak{P}}(z) \in z A[z]$. If $I$ is a non zero ideal of $O_{L}$, write $I=\prod_{j=1}^{t} \mathfrak{P}_{j}^{n_{j}}$ its decomposition into prime ideals of $O_{L}$, and write:

$$
f_{I}(z)=\prod_{j=1}^{t} f_{\mathfrak{P}_{j}}(z)^{n_{j}} \in A[z] .
$$

Then, we have:

$$
\mathcal{L}\left(\widetilde{\phi} / \widetilde{O_{L}}\right)=\sum_{I \neq\{0\}} \frac{f_{I}(z)}{N_{L / K}(I)} \in \mathbb{T}_{z}\left(K_{\infty}\right)^{\times}
$$

In particular:

$$
\begin{gathered}
\left.\mathcal{L}\left(\widetilde{\phi} / \widetilde{O_{L}}\right)\right|_{z=0}=1 \\
\left.\mathcal{L}\left(\widetilde{\phi} / \widetilde{O_{L}}\right)\right|_{z=1}=\mathcal{L}\left(\phi / O_{L}\right)=\sum_{I \neq\{0\}} \frac{f_{I}(1)}{N_{L / K}(I)} \in K_{\infty}^{\times} .
\end{gathered}
$$




\subsection{Stark units for Drinfeld modules.}

Let $\phi: A \rightarrow O_{L}\{\tau\}$ be a Drinfeld $A$-module. Let $e v: \mathbb{T}_{z}\left(L_{\infty}\right) \rightarrow L_{\infty}$ be the surjective morphism of $\mathbb{F}_{q}$-vector spaces given by $\forall f \in \mathbb{T}_{z}\left(L_{\infty}\right)$,ev $(f)=\left.f\right|_{z=1}$. We set:

$$
U_{S t}\left(\phi / O_{L}\right)=\operatorname{ev}\left(U\left(\widetilde{\phi} / O_{L}[z]\right)\right)
$$

We call $U_{S t}\left(\phi / O_{L}\right)$ the $A$-module of Stark units relative to $\phi$ and $O_{L}$. Since $e v\left(\exp _{\tilde{\phi}}\right)=\exp _{\phi}, U_{S t}\left(\phi / O_{L}\right)$ is a sub- $A$-module of $U\left(\phi / O_{L}\right)$.

Let $\alpha: \mathbb{T}_{z}\left(L_{\infty}\right) \rightarrow \mathbb{T}_{z}\left(L_{\infty}\right)$ be the morphism of $\mathbb{F}_{q}[z]$-modules given by:

$$
\forall x \in \mathbb{T}_{z}\left(L_{\infty}\right), \alpha(x)=\frac{\exp _{\tilde{\phi}}(x)-\exp _{\phi}(x)}{z-1} \in \mathbb{T}_{z}\left(L_{\infty}\right) .
$$

Recall that $H\left(\widetilde{\phi} / O_{L}[z]\right)$ is an $A[z]$-module via $\widetilde{\phi}$. For $f \in A[z]$, we set

$$
H\left(\widetilde{\phi} / O_{L}[z]\right)[f]=\left\{x \in H\left(\widetilde{\phi} / O_{L}[z]\right), f x=0\right\} .
$$

Proposition 2.6. The map $\alpha$ induces an isomorphism of A-modules:

$$
\bar{\alpha}: \frac{U\left(\phi / O_{L}\right)}{U_{S t}\left(\phi / O_{L}\right)} \simeq H\left(\widetilde{\phi} / O_{L}[z]\right)[z-1] .
$$

Proof.

Let $x \in U\left(\phi / O_{L}\right)$, then:

$$
(z-1) \alpha(x)=\exp _{\tilde{\phi}}(x)-\exp _{\phi}(x) \in O_{L}+\exp _{\widetilde{\phi}}\left(\mathbb{T}_{z}\left(L_{\infty}\right)\right) .
$$

Therefore $(z-1) \alpha(x)=0$ in $H\left(\widetilde{\phi} / O_{L}[z]\right)$, i.e. the image $\alpha(x)$ in $H\left(\widetilde{\phi} / O_{L}[z]\right)$ lies in $H\left(\widetilde{\phi} / O_{L}[z]\right)[z-1]$. Furthermore:

$$
\forall x \in \mathbb{T}_{z}\left(L_{\infty}\right), \alpha(\theta x)=\widetilde{\phi}_{\theta}(\alpha(x))+\left(\sum_{j=1}^{r} \alpha_{j} \frac{z^{j}-1}{z-1} \tau^{j}\right)\left(\exp _{\phi}(x)\right),
$$

where $\phi_{\theta}=\sum_{j=0}^{r} \alpha_{j} \tau^{j}, \alpha_{j} \in O_{L}$. Thus $\alpha$ induces a morphism of $A$-modules $\bar{\alpha}$ : $U\left(\phi / O_{L}\right) \rightarrow H\left(\widetilde{\phi} / O_{L}[z]\right)[z-1]$.

Let $x \in \mathbb{T}_{z}\left(L_{\infty}\right)$ such that the image of $x$ in $H\left(\widetilde{\phi} / O_{L}[z]\right)$ lies in $H\left(\widetilde{\phi} / O_{L}[z]\right)[z-1]$. Then:

$$
(z-1) x=a+\exp _{\widetilde{\phi}}(h), a \in O_{L}[z], h \in \mathbb{T}_{z}\left(L_{\infty}\right) .
$$

Write $a=b+(z-1) c, b \in O_{L}, c \in O_{L}[z], h=g+(z-1) v, g \in L_{\infty}, v \in \mathbb{T}_{z}\left(L_{\infty}\right)$. Then:

$$
0=b+\exp _{\phi}(g)
$$

Thus:

$$
b=-\exp _{\phi}(g) \in O_{L} .
$$

Thus $g \in U\left(\phi / O_{L}\right)$. We get:

$$
(z-1)\left(x-c-\exp _{\widetilde{\phi}}(v)\right)=\exp _{\widetilde{\phi}}(g)-\exp _{\phi}(g)=(z-1) \alpha(g) .
$$

Thus the image of $x$ in $H\left(\widetilde{\phi} / O_{L}[z]\right)$ is equal to the image of $\alpha(g)$ in $H\left(\widetilde{\phi} / O_{L}[z]\right)$. This implies that $\bar{\alpha}: U\left(\phi / O_{L}\right) \rightarrow H\left(\widetilde{\phi} / O_{L}[z]\right)[z-1]$ is a surjective morphism of $A$-modules.

Let $x \in U_{S t}\left(\phi / O_{L}\right)$. Then, there exist $u \in U\left(\widetilde{\phi} / O_{L}[z]\right)$ and $h \in \mathbb{T}_{z}\left(L_{\infty}\right)$ such that:

$$
x=u+(z-1) h .
$$


We get:

$$
\exp _{\widetilde{\phi}}(x)=\exp _{\widetilde{\phi}}(u)+(z-1) \exp _{\widetilde{\phi}}(h) .
$$

Since $\exp _{\widetilde{\phi}}(u) \in O_{L}[z]$ and $e v\left(\exp _{\widetilde{\phi}}(u)\right)=\exp _{\phi}(x) \in O_{L}$, we get:

$$
\begin{aligned}
\exp _{\widetilde{\phi}}(x)-\exp _{\phi}(x) & =\left(\exp _{\widetilde{\phi}}(u)-\exp _{\phi}(x)\right)+(z-1) \exp _{\widetilde{\phi}}(h) \\
& \in(z-1)\left(O_{L}[z]+\exp _{\widetilde{\phi}}\left(\mathbb{T}_{z}\left(L_{\infty}\right)\right)\right),
\end{aligned}
$$

i.e. :

$$
\alpha(x) \in O_{L}[z]+\exp _{\widetilde{\phi}}\left(\mathbb{T}_{z}\left(L_{\infty}\right)\right) .
$$

Thus $U_{S t}\left(\phi / O_{L}\right) \subset \operatorname{Ker} \bar{\alpha}$.

Now, let $x \in U\left(\phi / O_{L}\right)$ such that $\alpha(x) \in O_{L}[z]+\exp _{\widetilde{\phi}}\left(\mathbb{T}_{z}\left(L_{\infty}\right)\right)$. Then:

$$
\exp _{\widetilde{\phi}}(x) \in O_{L}[z]+\exp _{\widetilde{\phi}}\left((z-1) \mathbb{T}_{z}\left(L_{\infty}\right)\right) .
$$

Thus $x \in U\left(\widetilde{\phi} / O_{L}[z]\right)+(z-1) \mathbb{T}_{z}\left(L_{\infty}\right)$. Thus:

$$
x=e v(x) \in \operatorname{ev}\left(U\left(\widetilde{\phi} / O_{L}[z]\right)\right)=U_{S t}\left(\phi / O_{L}\right) .
$$

Therefore Ker $\bar{\alpha}=U_{S t}\left(\phi / O_{L}\right)$.

Theorem 2.7. $\frac{U\left(\phi / O_{L}\right)}{U_{S t}\left(\phi / O_{L}\right)}$ is a finite A-module and:

$$
\left[H\left(\phi / O_{L}\right)\right]_{A}=\left[\frac{U\left(\phi / O_{L}\right)}{U_{S t}\left(\phi / O_{L}\right)}\right]_{A} .
$$

In particular:

$$
\mathcal{L}\left(\phi / O_{L}\right)=\left[O_{L}: U_{S t}\left(\phi / O_{L}\right)\right]_{A}
$$

Proof.

By Proposition 2.4 $H\left(\widetilde{\phi} / O_{L}[z]\right)$ is a finite dimensional $\mathbb{F}_{q}$-vector space, thus a finite $A$-module (via $\widetilde{\phi})$. In particular $H\left(\widetilde{\phi} / O_{L}[z]\right)[z-1]$ is a finite $A$-module. Thus Proposition 2.6 implies that $\frac{U\left(\phi / O_{L}\right)}{U_{S t}\left(\phi / O_{L}\right)}$ is a finite $A$-module.

Observe that the map $e v$ induces an exact sequence of $A$-modules:

$$
0 \rightarrow(z-1) H\left(\widetilde{\phi} / O_{L}[z]\right) \rightarrow H\left(\widetilde{\phi} / O_{L}[z]\right) \rightarrow H\left(\phi / O_{L}\right) \rightarrow 0 .
$$

Therefore the multiplication by $z-1$ on $H\left(\widetilde{\phi} / O_{L}[z]\right)$ gives rise to an exact sequence of finite $A$-modules:

$$
0 \rightarrow H\left(\widetilde{\phi} / O_{L}[z]\right)[z-1] \rightarrow H\left(\widetilde{\phi} / O_{L}[z]\right) \rightarrow H\left(\widetilde{\phi} / O_{L}[z]\right) \rightarrow H\left(\phi / O_{L}\right) \rightarrow 0 .
$$

This implies that (recall that if $0 \rightarrow M_{2} \rightarrow M_{1} \rightarrow M_{3} \rightarrow 0$ is an exact sequence of finite $A$-modules then $\left.\left[M_{1}\right]_{A}=\left[M_{2}\right]_{A}\left[M_{3}\right]_{A}\right)$ :

$$
\left[H\left(\widetilde{\phi} / O_{L}[z]\right)[z-1]\right]_{A}=\left[H\left(\phi / O_{L}\right)\right]_{A} .
$$

It remains to apply Proposition 2.6 and formula (2).

Lemma 2.8. Let $\phi$ be an $A$-Drinfeld module defined over $O_{L}$. Let $E / L$ be a finite extension. The inclusion $L \subset E$ induces a natural injective morphism of $K_{\infty}$ algebras: $\iota_{E / L}: L_{\infty} \hookrightarrow E_{\infty}$. Then:

1) $\iota_{E / L}\left(U_{S t}\left(\phi / O_{L}\right)\right) \subset U_{S t}\left(\phi / O_{E}\right)$;

2) $U_{S t}\left(\phi / O_{E}\right) \cap \iota_{E / L}\left(L_{\infty}\right)=\iota_{E / L}\left(U_{S t}\left(\phi / O_{L}\right)\right)$;

3) $\operatorname{Tr}_{E / L}\left(U_{S t}\left(\phi / O_{E}\right)\right) \subset U_{S t}\left(\phi / O_{L}\right)$. 
Proof. Let's denote the natural morphism of $\mathbb{T}_{z}\left(K_{\infty}\right)$-algebras induced by the inclusion $L \subset E$ by $\iota_{E / L}^{\prime}: \mathbb{T}_{z}\left(L_{\infty}\right) \hookrightarrow \mathbb{T}_{z}\left(E_{\infty}\right)$. Then:

$$
\iota_{E / L}^{\prime}\left(U\left(\widetilde{\phi} / O_{L}[z]\right)\right) \subset U\left(\widetilde{\phi} / O_{E}[z]\right) .
$$

Since $e v \circ \iota_{E / L}^{\prime}=\iota_{E / L} \circ \mathrm{ev}$, we get 1). Let $\operatorname{Tr}_{E / L}: E \rightarrow L$ be the trace map relative to the extension $E / L$. Then $T r_{E / L}$ induces a continuous morphism of $K_{\infty}$-algebras still denoted by $\operatorname{Tr}_{E / L}: E_{\infty} \rightarrow L_{\infty}$ and a continuous morphism of $\mathbb{T}_{z}\left(K_{\infty}\right)$-algebras $\operatorname{Tr}_{E / L}^{\prime}: \mathbb{T}_{z}\left(E_{\infty}\right) \rightarrow \mathbb{T}_{z}\left(L_{\infty}\right)$. Observe that:

$$
e v \circ \operatorname{Tr}_{E / L}^{\prime}=\operatorname{Tr}_{E / L} \circ \mathrm{ev} .
$$

For $x \in \mathbb{T}_{z}\left(E_{\infty}\right)$, we have:

$$
\operatorname{Tr}_{E / L}^{\prime}\left(\exp _{\tilde{\phi}}(x)\right)=\exp _{\widetilde{\phi}}\left(\operatorname{Tr}_{E / L}^{\prime}(x)\right)
$$

Since $\operatorname{Tr}_{E / L}^{\prime}\left(O_{E}[z]\right) \subset O_{L}[z]$, we get :

$$
\operatorname{Tr}_{E / L}^{\prime}\left(U\left(\widetilde{\phi} / O_{E}[z]\right)\right) \subset U\left(\widetilde{\phi} / O_{L}[z]\right)
$$

Thus we get 3). Now, we observe that:

$$
O_{E}[z] \cap \iota_{E / L}^{\prime}\left(\mathbb{T}_{z}\left(L_{\infty}\right)\right)=\iota_{E / L}^{\prime}\left(O_{L}[z]\right) .
$$

This is an easy consequence of the following fact: let $e_{1}, \ldots, e_{k}, \ldots e_{n}$ be a basis of $E$ over $K$ where $e_{1}, \ldots, e_{k}$ is a basis of $L$. Then one can write any $x \in \widetilde{E}_{\infty}$ in a unique way as $x=\sum_{i=1}^{n} e_{i} \otimes \lambda_{i}$ with $\lambda_{i} \in \widetilde{K}_{\infty}$. This implies:

$$
U\left(\widetilde{\phi} / O_{E}[z]\right) \cap \iota_{E / L}^{\prime}\left(\mathbb{T}_{z}\left(L_{\infty}\right)\right)=\iota_{E / L}^{\prime}\left(U\left(\widetilde{\phi} / O_{L}[z]\right)\right) .
$$

Therefore, we get 2).

Corollary 2.9. Let $\phi$ be a Drinfeld A-module defined over $O_{L}$. let $E / L$ be a finite extension. Then:

$$
\frac{\left[H\left(\phi / O_{E}\right)\right]_{A}}{\left[H\left(\phi / O_{L}\right)\right]_{A}} \in A
$$

Furthermore $\frac{O_{E}}{\iota_{E / L}\left(O_{L}\right)}$ and $\frac{U_{S t}\left(\phi / O_{E}\right)}{\iota_{E / L}\left(U_{S t}\left(\phi / O_{L}\right)\right)}$ are two A-lattices in the finite dimensional $K_{\infty}$-vector space $\frac{E_{\infty}}{\iota_{E / L}\left(L_{\infty}\right)}$ and we have:

$$
\left[\frac{O_{E}}{\iota_{E / L}\left(O_{L}\right)}: \frac{U_{S t}\left(\phi / O_{E}\right)}{\iota_{E / L}\left(U_{S t}\left(\phi / O_{L}\right)\right)}\right]_{A}=\frac{\mathcal{L}\left(\phi / O_{E}\right)}{\mathcal{L}\left(\phi / O_{L}\right)} .
$$

Proof. By Lemma 2.8, $\iota_{E / L}$ induces an injective morphism of $A$-modules:

$$
\frac{U\left(\phi / O_{L}\right)}{U_{S t}\left(\phi / O_{L}\right)} \hookrightarrow \frac{U\left(\phi / O_{E}\right)}{U_{S t}\left(\phi / O_{E}\right)}
$$

By Theorem 2.7, the above $A$-modules are finite, thus:

$$
\frac{\left[\frac{U\left(\phi / O_{E}\right)}{U_{S t}\left(\phi / O_{E}\right)}\right]_{A}}{\left[\frac{U\left(\phi / O_{L}\right)}{U_{S t}\left(\phi / O_{L}\right)}\right]_{A}} \in A
$$

Applying again Theorem 2.7 we get:

$$
\frac{\left[H\left(\phi / O_{E}\right)\right]_{A}}{\left[H\left(\phi / O_{L}\right)\right]_{A}} \in A .
$$


Furthermore, by (4), we also have:

$$
\left[\frac{U\left(\phi / O_{E}\right)}{U_{S t}\left(\phi / O_{E}\right)+\iota_{E / L}\left(U\left(\phi / O_{L}\right)\right)}\right]_{A}=\frac{\left[H\left(\phi / O_{E}\right)\right]_{A}}{\left[H\left(\phi / O_{L}\right)\right]_{A}} .
$$

Observe that $O_{E} \cap \iota_{E / L}\left(L_{\infty}\right)=\iota_{E / L}\left(O_{L}\right)$. Now $\frac{O_{E}}{\iota_{E / L}\left(O_{L}\right)}$ is an $A$-lattice in $\frac{E_{\infty}}{\iota_{E / L}\left(L_{\infty}\right)}$. By Lemma 2.8 and Theorem 2.7, we also have that $\frac{U_{S t}\left(\phi / O_{E}\right)}{\iota_{E / L}\left(U_{S t}\left(\phi / O_{L}\right)\right)}$ is an $A$-lattice in $\frac{E_{\infty}}{\iota_{E / L}\left(L_{\infty}\right)}$. We have:

$$
\left[\frac{O_{E}}{\iota_{E / L}\left(O_{L}\right)}: \frac{U_{S t}\left(\phi / O_{E}\right)}{\iota_{E / L}\left(U_{S t}\left(\phi / O_{L}\right)\right)}\right]_{A}=\frac{\left[O_{E}: U_{S t}\left(\phi / O_{E}\right)\right]_{A}}{\left[O_{L}: U_{S t}\left(\phi / O_{L}\right)\right]_{A}} .
$$

It remains to apply Theorem 2.7 .

Lemma 2.10. Let $\phi$ be a Drinfeld $A$-module defined over $O_{L}$. Let $E / L$ be a finite Galois extension and let $G=\operatorname{Gal}(E / L)$. Then $U\left(\phi / O_{E}\right)$ and $U_{S t}\left(\phi / O_{E}\right)$ are $A[G]$ modules and:

$$
\begin{aligned}
U\left(\phi / O_{E}\right)^{G} & =\iota_{E / L}\left(U\left(\phi / O_{L}\right)\right), \\
U_{S t}\left(\phi / O_{E}\right)^{G} & =\iota_{E / L}\left(U_{S t}\left(\phi / O_{L}\right)\right) .
\end{aligned}
$$

Proof. We prove the assertion for Stark units. Observe that $\exp _{\tilde{\phi}}: \mathbb{T}_{z}\left(E_{\infty}\right) \rightarrow$ $\mathbb{T}_{z}\left(E_{\infty}\right)$ is a morphism of $\mathbb{F}_{q}[z][G]$-modules. Thus $U\left(\widetilde{C} / O_{E}[z]\right)$ is an $A[z][G]$ module. Since ev $: \mathbb{T}_{z}\left(E_{\infty}\right) \rightarrow E_{\infty}$ is a morphism of $A[G]$-modules, we deduce that $U_{S t}\left(\phi / O_{E}\right)$ is a $A[G]$-module. Now, observe that:

$$
E_{\infty}^{G}=\iota_{E / L}\left(L_{\infty}\right)
$$

Therefore, by Lemma 2.8 , we get:

$$
U_{S t}\left(\phi / O_{E}\right)^{G}=U_{s t}\left(\phi / O_{E}\right) \cap \iota_{E / L}\left(L_{\infty}\right)=\iota_{E / L}\left(U_{S t}\left(\phi / O_{L}\right)\right) .
$$

Proposition 2.11. Let $\varphi$ be a Drinfeld $\widetilde{A}$-module defined over $\widetilde{O_{L}}$. Let $E / L$ be a finite abelian extension of degree prime to the characteristic of $\mathbb{F}_{q}$, and let $G=$ $\operatorname{Gal}(E / L)$. Then:

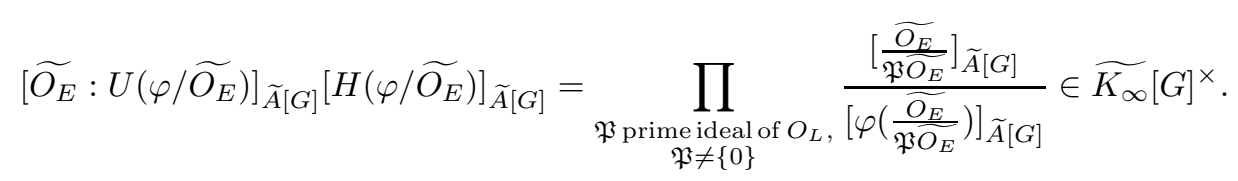

Proof. The above result is a consequence of the proof of [7, Theorem A. We refer the reader to [27, 7], 9], 10, for the background on the class number formula. We only give a sketch of the proof. We also suspect that this equivariant formula can also be deduced from [11, working over $\mathbb{F}_{q}(z)$ instead of working over $\mathbb{F}_{q}$.

Recall that $O_{E}$ is a free $A[G]$-module. Set $k=\mathbb{F}_{q}(z)$. Let $F / k$ be the finite extension obtained by adjoining to $k$ the elements $\chi(g), \forall \chi \in \widehat{G}, \forall g \in G$.

We have an exact sequence of $\widetilde{A}[G]$-modules (the module in the center is a $\widetilde{A}$ module via $\varphi$ ):

$$
O \rightarrow \frac{\widetilde{E_{\infty}}}{U\left(\varphi / \widetilde{O_{E}}\right)} \rightarrow \frac{\widetilde{E_{\infty}}}{\widetilde{O_{E}}} \rightarrow H\left(\varphi / \widetilde{O_{E}}\right) \rightarrow 0
$$


Since $\frac{\widetilde{E_{\infty}}}{U\left(\varphi / O_{E}\right)}$ is a divisible $\widetilde{A}[G]$-module and $\widetilde{A}[G]$ is a principal ideal ring, the above sequence of $\widetilde{A}[G]$-modules splits. We obtain an isomorphism of $\widetilde{A}[G]$-modules:

$$
\gamma: \frac{\widetilde{E_{\infty}}}{U\left(\varphi / \widetilde{O_{E}}\right)} \times H\left(\varphi / \widetilde{O_{E}}\right) \simeq \frac{\widetilde{E_{\infty}}}{\widetilde{O_{E}}} .
$$

We still denote by $\tau$ the continuous morphism of $F(z)$-algebras: $\tau \otimes 1: \widetilde{E_{\infty}} \otimes_{k} F \rightarrow$ $\widetilde{E_{\infty}} \otimes_{k} F$. Let $\chi \in \widehat{G}$, by [7], proof of Theorem A and [9], Proposition 3.7, we deduce that

$$
\left[e_{\chi}\left(H\left(\varphi / \widetilde{O_{E}}\right) \otimes_{k} F\right)\right]_{F[\theta]}\left[e_{\chi}\left(\widetilde{O_{E}} \otimes_{k} F\right): e_{\chi}\left(U\left(\varphi / \widetilde{O_{E}}\right) \otimes_{k} F\right)\right]_{F[\theta]}
$$

is equal to

$$
\left.\operatorname{det}_{F[[Z]]}\left(1+\Theta \mid \frac{\widetilde{L_{\infty}} \otimes_{\widetilde{A}} e_{\chi}\left(\widetilde{O_{E}} \otimes_{k} F\right)}{e_{\chi}\left(\widetilde{O_{E}} \otimes_{k} F\right)}\right)\right|_{Z=\theta^{-1}}
$$

where we refer the reader to 9 , section 3.1, for the definition of $\operatorname{det}_{F[[Z]]}($.$) , and$ where :

$$
\Theta=\sum_{n \geq 1}\left(\theta-\varphi_{\theta}\right) \theta^{n-1} Z^{n} \in \widetilde{O_{L}}\{\tau\}[[Z]] .
$$

Now, observe that $e_{\chi}\left(\widetilde{O_{E}} \otimes_{k} F\right)$ is a finitely generated and projective $\widetilde{O_{L}}$-module. Therefore, by an adaptation of [27], Theorem 3, similar to that made in [9], and by [7, paragraph 6.4 , if we set $M=e_{\chi}\left(\widetilde{O_{E}} \otimes_{k} F\right)$, we get:

$$
\operatorname{det}_{F[Z]]}\left(1+\Theta \mid \frac{\widetilde{L_{\infty}} \otimes_{\widetilde{A}} M}{M}\right)=\prod_{\mathfrak{P}} \operatorname{det}_{F[Z]]}\left(1+\Theta \mid \frac{M}{\mathfrak{P} M}\right)^{-1},
$$

where the product is over the maximal ideals of $O_{L}$. But observe that:

$$
\prod_{\mathfrak{P}}\left(\left.\operatorname{det}_{F[Z]]}\left(1+\Theta \mid \frac{M}{\mathfrak{P} M}\right)\right|_{Z=\theta^{-1}}\right)^{-1}=\prod_{\mathfrak{P}} \frac{\left[\frac{M}{\mathfrak{P} M}\right]_{F[\theta]}}{\left[\varphi\left(\frac{M}{\mathfrak{P} M}\right)\right]_{F[\theta]}} .
$$

Thus, we get the desired equivariant formula. The reader will notice that the above reasoning works also for Drinfeld $A$-modules leading to Fang's relative equivariant class number formula (11, Theorem 1.13).

Corollary 2.12. Let $\phi$ be a Drinfeld $A$-module defined over $O_{L}$. Let $E / L$ be a finite abelian extension of degree prime to the characteristic of $\mathbb{F}_{q}$, and let $G=\operatorname{Gal}(E / L)$. Then:

We also have:

$$
\left[\frac{U\left(\phi / O_{E}\right)}{U_{S t}\left(\phi / O_{E}\right)}\right]_{A[G]}=\left[H\left(\phi / O_{E}\right)\right]_{A[G]}
$$

$$
\left[O_{E}: U_{S t}\left(\phi / O_{E}\right)\right]_{A[G]}=\prod_{\mathfrak{P} \text { prime ideal of } O_{L}, \mathfrak{P} \neq\{0\}} \frac{\left[\frac{O_{E}}{\mathfrak{P} O_{E}}\right]_{A[G]}}{\left[\phi\left(\frac{O_{E}}{\mathfrak{P} O_{E}}\right)\right]_{A[G]}} \in K_{\infty}[G]^{\times} .
$$

Proof. By Lemma 2.10, we have that $U\left(\phi / O_{E}\right)$ and $U_{S t}\left(\phi / O_{E}\right)$ are $A[G]$-lattices in $E_{\infty}$. Now, observe that the map $\bar{\alpha}$ of Proposition 2.6] induces an isomorphism of $A[G]$-modules:

$$
\frac{U\left(\phi / O_{E}\right)}{U_{S t}\left(\phi / O_{E}\right)} \simeq H\left(\widetilde{\phi} / O_{E}[z]\right)[z-1]
$$


BRUNO ANGLÈS AND FLORIC TAVARES RIBEIRO

Thus, using similar arguments as that used in the proof of Theorem 2.7, we get the first assertion. The second assertion is a consequence of the first assertion and [1], Theorem 1.13 (see also the proof of Proposition 2.11).

Let $\phi$ be a Drinfeld $A$-module defined over $O_{L}$. Let $P$ be a monic irreducible element in $A$, and let $\mathfrak{P}_{1}, \ldots, \mathfrak{P}_{t}$ be the distinct maximal ideals of $O_{L}$ above $P$. Write $P O_{L}=\mathfrak{P}_{1}^{e_{1}} \cdots \mathfrak{P}_{t}^{e_{t}}$. Then we have an exact sequence of $A$-modules:

$$
0 \rightarrow \prod_{j=1}^{t} \frac{\mathfrak{P}_{j}}{\mathfrak{P}_{j}^{e_{j}}} \rightarrow \frac{O_{L}}{P O_{L}} \rightarrow \prod_{j=1}^{t} \frac{O_{L}}{\mathfrak{P}_{j}} \rightarrow 0
$$

Now, observe that $\forall n \geq 1$, for $j=1, \ldots, t, \tau\left(\mathfrak{P}_{j}^{n}\right) \subset \mathfrak{P}_{j}^{n+1}$. This implies:

$$
\frac{\left[\frac{O_{L}}{P O_{L}}\right]_{A}}{\left[\phi\left(\frac{O_{L}}{P O_{L}}\right)\right]_{A}}=\prod_{j=1}^{t} \frac{\left[\frac{O_{L}}{\mathfrak{P}_{j}}\right]_{A}}{\left[\phi\left(\frac{O_{L}}{\mathfrak{P}_{j}}\right)\right]_{A}}
$$

Therefore:

$$
\mathcal{L}\left(\phi / O_{L}\right)=\prod_{P} \frac{\left[\frac{O_{L}}{P O_{L}}\right]_{A}}{\left[\phi\left(\frac{O_{L}}{P O_{L}}\right)\right]_{A}}
$$

where $P$ runs through the monic irreducible elements in $A$. Now let $E / L$ be a finite abelian extension of degree prime to the characteristic of $\mathbb{F}_{q}$. Then, the above discussion implies:

$$
\mathcal{L}\left(\phi /\left(O_{E} / O_{L}\right), G\right):=\prod_{\mathfrak{P} \text { prime ideal of } O_{L}, \mathfrak{P} \neq\{0\}} \frac{\left[\frac{O_{E}}{\mathfrak{P} O_{E}}\right]_{A[G]}}{\left[\phi\left(\frac{O_{E}}{\mathfrak{P} O_{E}}\right)\right]_{A[G]}}=\prod_{P} \frac{\left[\frac{O_{E}}{P O_{E}}\right]_{A[G]}}{\left[\phi\left(\frac{O_{E}}{P O_{E}}\right)\right]_{A[G]}}
$$

Let $P$ be a monic irreducible element in $A$, since $O_{E}$ is a free $A[G]$-module, we have:

$$
\begin{gathered}
{\left[\frac{O_{E}}{P O_{E}}\right]_{A[G]}=P^{[L: K]} 1_{G},} \\
{\left[\phi\left(\frac{O_{E}}{P O_{E}}\right)\right]_{A[G]}=\operatorname{det}_{\mathbb{F}_{q}[G][Z]}\left((1 \otimes Z) \mathrm{Id}-\left.\phi_{\theta} \otimes 1\right|_{\frac{O_{E}}{P O_{E}} \otimes_{\mathbb{F}_{q}} \mathbb{F}_{q}[Z]}\right)_{Z=\theta} .}
\end{gathered}
$$

If $\widetilde{\phi}$ is the canonical $z$-deformation of $\phi$, then:

$$
\mathcal{L}\left(\widetilde{\phi} / \widetilde{O_{L}}\right)=\prod_{P} \frac{\left[\frac{O_{L}[z]}{P O_{L}[z]}\right]_{A[z]}}{\left[\phi\left(\frac{O_{L}[z]}{P O_{L}[z]}\right)\right]_{A[z]}}
$$

where, for $P$ a monic irreducible prime of $A$, we have:

$$
\begin{gathered}
{\left[\frac{O_{L}[z]}{P O_{L}[z]}\right]_{A[z]}=P^{[L: K]}} \\
{\left[\phi\left(\frac{O_{L}[z]}{P O_{L}[z]}\right)\right]_{A[z]}=\operatorname{det}_{\mathbb{F}_{q}[z][Z]}\left((1 \otimes Z) \mathrm{Id}-\left.\phi_{\theta} \otimes 1\right|_{\frac{O_{L}[z]}{P O_{L}[z]} \otimes_{\mathbb{F}_{q}} \mathbb{F}_{q}[Z]}\right)_{Z=\theta} .}
\end{gathered}
$$

And also:

$$
\mathcal{L}\left(\widetilde{\phi} /\left(\widetilde{O_{E}} / \widetilde{O_{L}}\right), G\right):=\prod_{\mathfrak{P} \text { prime ideal of } O_{L}, \mathfrak{P} \neq\{0\}} \frac{\left[\widetilde{\widetilde{O_{E}}}\right.}{\left[\widetilde{\phi}\left(\frac{\widetilde{O_{E}}}{\mathfrak{P} \widetilde{O_{E}}}\right)\right]_{\widetilde{A}[G]}}=\prod_{P} \frac{\left[\frac{O_{E}[z]}{P O_{E}[z]}\right]_{A[z][G]}}{\left[\widetilde{\phi}\left(\frac{O_{E}[z]}{P O_{E}[z]}\right)\right]_{A[z][G]}}
$$

where, for a monic irreducible element $P$ of $A$, we have:

$$
\left[\frac{O_{E}[z]}{P O_{E}[z]}\right]_{A[z][G]}=P^{[L / K]} 1_{G}
$$




$$
\left[\widetilde{\phi}\left(\frac{O_{E}[z]}{P O_{E}[z]}\right)\right]_{A[z][G]}=\operatorname{det}_{\mathbb{F}_{q}[G][z, Z]}\left((1 \otimes Z) \operatorname{Id}-\left.\widetilde{\phi}_{\theta} \otimes 1\right|_{\frac{O_{E}[z]}{P O_{E}[z]} \otimes_{\mathbb{F}_{q}} \mathbb{F}_{q}[Z]}\right)_{Z=\theta} .
$$

In particular, since $\mathcal{L}\left(\widetilde{\phi} /\left(\widetilde{O_{E}} / \widetilde{O_{L}}\right), G\right)$ converges in $\widetilde{K_{\infty}}[G]$, we get that:

$$
\mathcal{L}\left(\widetilde{\phi} /\left(\widetilde{O_{E}} / \widetilde{O_{L}}\right), G\right) \in\left(\mathbb{T}_{z}\left(K_{\infty}\right)[G]\right)^{\times} .
$$

Theorem 2.13. Let $\phi$ be a Drinfeld $A$-module defined over $A$. Let $E / K$ be a finite abelian extension of degree prime to the characteristic of $\mathbb{F}_{q}$. Let $G=\operatorname{Gal}(E / K)$. Then:

In particular:

$$
\mathcal{L}\left(\widetilde{\phi} /\left(\widetilde{O_{E}} / \widetilde{A}\right), G\right) O_{E}[z]=U\left(\widetilde{\phi} / O_{E}[z]\right)
$$

$$
U_{S t}\left(\phi / O_{E}\right)=\mathcal{L}\left(\phi /\left(O_{E} / A\right), G\right) O_{E}
$$

Proof. Since $O_{E}$ is a free $A[G]$-module of rank one, we observe that $\widetilde{O_{E}}$ is a free $\widetilde{A}[G]$-module of rank one. It is also the case for $U\left(\widetilde{\phi} / \widetilde{O_{E}}\right)$. Write:

$$
\widetilde{O_{E}}=\widetilde{A}[G] \eta
$$

for some $\eta \in O_{E}$, and:

$$
U\left(\widetilde{\phi} / \widetilde{O_{E}}\right)=\widetilde{A}[G] \varepsilon
$$

for some $\varepsilon \in U\left(\widetilde{\phi} / O_{E}[z]\right)$ (we can find such an element by Proposition 2.3). Then:

$$
\widetilde{E_{\infty}}=\widetilde{K_{\infty}}[G] \eta=\widetilde{K_{\infty}}[G] \varepsilon
$$

This implies that there exists $f \in\left(\widetilde{K_{\infty}}[G]\right)^{\times}$such that:

$$
\varepsilon=f \eta \text {. }
$$

We have:

$$
\mathbb{T}_{z}\left(E_{\infty}\right)=\mathbb{T}_{z}\left(K_{\infty}\right)[G] \eta
$$

Thus $f \in \mathbb{T}_{z}\left(K_{\infty}\right)[G]$. Furthermore, by Proposition 2.11, we have:

$$
\mathcal{L}\left(\widetilde{\phi} /\left(\widetilde{O_{E}} / \widetilde{A}\right), G\right)=\delta f
$$

for some $\delta \in\left(\mathbb{F}_{q}(z)[G]\right)^{\times}$. But we have that $\mathcal{L}\left(\widetilde{\phi} /\left(\widetilde{O_{E}} / \widetilde{A}\right), G\right) \in\left(\mathbb{T}_{z}\left(K_{\infty}\right)[G]\right)^{\times}$. This implies that $x:=\frac{1}{\delta} \in \mathbb{F}_{q}[z][G]$. We get:

$$
\exp _{\widetilde{\phi}}(\varepsilon) \in x \mathbb{T}_{z}\left(E_{\infty}\right) \cap O_{E}[z]=x O_{E}[z]
$$

The above equality comes from the fact that $x \mathbb{T}_{z}\left(K_{\infty}\right)[G] \cap A[z][G]=x A[z][G]$. Set:

then:

$$
u=\frac{1}{x} \varepsilon=\delta \varepsilon \in U\left(\widetilde{\phi} / \widetilde{O_{E}}\right),
$$

We have:

$$
\exp _{\widetilde{\phi}}(u) \in O_{E}[z] \text {. }
$$

$$
u=\mathcal{L}\left(\widetilde{\phi} /\left(\widetilde{O_{E}} / \widetilde{A}\right), G\right) \eta \in \mathbb{T}_{z}\left(E_{\infty}\right)
$$

Thus:

$$
u \in U\left(\widetilde{\phi} / O_{E}[z]\right) .
$$

Therefore:

$$
\mathcal{L}\left(\widetilde{\phi} /\left(\widetilde{O_{E}} / \widetilde{A}\right), G\right) O_{E}[z]=A[z][G] u \subset U\left(\widetilde{\phi} / O_{E}[z]\right) .
$$

This also implies that:

$$
\mathbb{T}_{z}\left(E_{\infty}\right)=\mathbb{T}_{z}\left(K_{\infty}\right)[G] u
$$


Now, let $m \in U\left(\widetilde{\phi} / O_{E}[z]\right)$. Since $U\left(\widetilde{\phi} / \widetilde{O_{E}}\right)=\widetilde{A}[G] u$, by Proposition 2.3. there exists $x \in \mathbb{F}_{q}[z] \backslash\{0\}$ such that $x m \in A[z][G] u$. But :

$$
A[z][G] \cap x \mathbb{T}_{z}\left(K_{\infty}\right)[G]=x A[z][G] .
$$

Thus $m \in A[z][G] u$. Therefore :

$$
U\left(\widetilde{\phi} / O_{E}[z]\right)=A[z][G] u=\mathcal{L}\left(\widetilde{\phi} /\left(\widetilde{O_{E}} / \widetilde{A}\right), G\right) O_{E}[z]
$$

\subsection{An example.}

Let $\widetilde{C}: \widetilde{A} \rightarrow \widetilde{A}\{\tau\}$ be the Drinfeld $\widetilde{A}$-module defined over $\widetilde{A}$ given by:

$$
\widetilde{C}_{\theta}=z \tau+\theta \text {. }
$$

Then:

$$
\exp _{\widetilde{C}}=\sum_{j \geq 0} \frac{z^{j}}{D_{j}} \tau^{j} \in K(z)\{\{\tau\}\},
$$

where $D_{0}=1$, and for $i \geq 1, D_{i}=\left(\theta^{q^{i}}-\theta\right) D_{i-1}^{q}$.

Let $L / K$ be a finite extension and let $E / L$ be a finite abelian extension of degree prime to the characteristic of $\mathbb{F}_{q}$. Let $G=\operatorname{Gal}(E / L)$. Let $\mathfrak{P}$ be a maximal ideal of $O_{L}$. Let $G_{\mathfrak{P}} \subset G$ be the decomposition group of $\mathfrak{P}$ in $E / L$. Fix $\mathfrak{M}$ a maximal ideal of $O_{E}$ above $\mathfrak{P}$. Le $I_{\mathfrak{P}}$ be the inertia group of $\mathfrak{P}$ in $E / L$, and let's define:

$$
\sigma_{\mathfrak{P}}=\frac{1}{\left|I_{\mathfrak{P}}\right|} \sum \delta \in \mathbb{F}_{q}\left[G_{\mathfrak{P}}\right]
$$

where the sum is over the elements $\delta \in G_{\mathfrak{P}}$ such that the image of $\delta$ in $\frac{G_{\mathfrak{P}}}{I_{\mathfrak{P}}}$ is the Frobenius of the extension $\frac{O_{E}}{\mathfrak{M}} / \frac{O_{L}}{\mathfrak{P}}$.

Lemma 2.14. Let $\mathfrak{P}$ be a non zero prime ideal of $O_{L}$ and let $P$ be the prime of $A$ such that $P A=\mathfrak{P} \cap A$. Write $\left[\frac{O_{L}}{\mathfrak{P}}\right]_{A}=P^{m}, m \geq 1$. Then:

$$
\begin{gathered}
{\left[\frac{\widetilde{O_{E}}}{\mathfrak{P} \widetilde{O_{E}}}\right]_{\widetilde{A}[G]}=P^{m} 1_{G},} \\
{\left[\widetilde{C}\left(\frac{\widetilde{O_{E}}}{\mathfrak{P} \widetilde{O_{E}}}\right)\right]_{\widetilde{A}[G]}=P^{m} 1_{G}-z^{m \operatorname{deg}_{\theta} P} \sigma_{\mathfrak{P}} .}
\end{gathered}
$$

Furthermore, if $\mathfrak{P}$ is unramified in $E$, then we have an isomorphism of $\widetilde{A}[G]$ modules:

$$
\widetilde{C}\left(\frac{\widetilde{O_{E}}}{\mathfrak{P} \widetilde{O_{E}}}\right) \simeq \frac{\widetilde{A}[G]}{\left(P^{m} 1_{G}-z^{m \operatorname{deg}_{\theta} P} \sigma_{\mathfrak{P}}\right) \widetilde{A}[G]} .
$$

Proof. The computation of equivariant Fitting ideals can be done by an adaptation of the results in [11, section 6 . However, we present here an alternative proof. Fix a maximal ideal $\mathfrak{M}$ of $O_{E}$ above $\mathfrak{P}$ and let $e$ be the ramification index of $\mathfrak{M} / \mathfrak{P}$. Then we have an isomorphism of $\widetilde{A}[G]$-modules:

$$
\frac{O_{E}}{\mathfrak{P} O_{E}} \simeq \frac{O_{E}}{\mathfrak{M}^{e}} \otimes_{\mathbb{F}_{q}\left[G_{\mathfrak{P}}\right]} \mathbb{F}_{q}[G]
$$

Observe that we have an isomorphism of $\widetilde{A}$-modules:

$$
\widetilde{O_{E}} \simeq O_{E}[z] \otimes_{\mathbb{F}_{q}[z]} \mathbb{F}_{q}(z) \text {. }
$$


Therefore, we have an isomorphism of $\widetilde{A}$-modules:

$$
\frac{\widetilde{O_{E}}}{\mathfrak{M}^{e}{\widetilde{O_{E}}}} \simeq \frac{O_{E}}{\mathfrak{M}^{e}}[z] \otimes_{\mathbb{F}_{q}[z]} \mathbb{F}_{q}(z)
$$

Now, by [24], $\frac{O_{E}}{\mathfrak{M}^{e}}[z]$ is a free $\frac{O_{L}}{\mathfrak{P}}[z]\left[G_{\mathfrak{P}}\right]$-module. Thus:

$$
\left[\frac{\widetilde{O_{E}}}{\mathfrak{P} \widetilde{O_{E}}}\right]_{\widetilde{A}[G]}=P^{m} 1_{G} \text {. }
$$

We observe that:

$$
\widetilde{C}_{P} \equiv z^{d} \tau^{d} \quad(\bmod P \widetilde{A}\{\tau\})
$$

where $d=\operatorname{deg}_{\theta} P$. Set:

$$
\overline{G_{\mathfrak{P}}}=\frac{G_{\mathfrak{P}}}{I_{\mathfrak{P}}} .
$$

The natural morphism $G_{\mathfrak{P}} \rightarrow \overline{G_{\mathfrak{P}}}$ induces a surjective morphism of $\mathbb{F}_{q}$-algebras $\mathbb{F}_{q}\left[G_{\mathfrak{P}}\right] \rightarrow \mathbb{F}_{q}\left[\overline{G_{\mathfrak{P}}}\right]$. If $x \in \mathbb{F}_{q}\left[G_{\mathfrak{P}}\right]$, we denote its image in $\mathbb{F}_{q}\left[\overline{G_{\mathfrak{P}}}\right]$ by $\bar{x}$. We have :

$$
\left(\widetilde{C}_{P^{m}}-z^{d m} \overline{\sigma_{\mathfrak{P}}}\right)\left(\frac{\widetilde{O_{E}}}{\mathfrak{M} \widetilde{O_{E}}}\right)=\{0\} .
$$

Furthermore, observe that:

$$
\left\{x \in \frac{\widetilde{O_{E}}}{\mathfrak{M} \widetilde{O_{E}}}, \tau(x)=x\right\}=\mathbb{F}_{q}(z) .
$$

Since $\frac{\widetilde{O_{E}}}{\mathfrak{M} O_{E}}$ is a free $\frac{\widetilde{O_{L}}}{\mathfrak{P} O_{L}}\left[\overline{G_{\mathfrak{P}}}\right]$-module of rank one and $m d=\operatorname{dim}_{\mathbb{F}_{q}(z)} \frac{\widetilde{O_{L}}}{\mathfrak{P} O_{L}}$, by an adaptation to our case of the proof of [5], Lemma 5.7, and the proof of [5], Lemma 5.8 , we deduce that there exists a finite extension $F / \mathbb{F}_{q}$ (simply take the finite extension of $\mathbb{F}_{q}$ obtained by adjoining the values of the characters of $\left.\overline{G_{\mathfrak{P}}}\right)$ such that we have an isomorphism of $\widetilde{A}\left[\overline{G_{\mathfrak{P}}}\right] \otimes_{\mathbb{F}_{q}} F$-modules:

$$
\widetilde{C}\left(\frac{\widetilde{O_{E}}}{\mathfrak{M} \widetilde{O_{E}}}\right) \otimes_{\mathbb{F}_{q}} F \simeq \frac{\widetilde{A}\left[\overline{G_{\mathfrak{P}}}\right] \otimes_{\mathbb{F}_{q}} F}{\left(\left(P^{m} 1_{\overline{G_{\mathfrak{P}}}}-z^{d m} \overline{\sigma_{\mathfrak{P}}}\right) \otimes 1\right) \widetilde{A}\left[\overline{G_{\mathfrak{P}}}\right] \otimes_{\mathbb{F}_{q}} F} .
$$

Therefore, we have an isomorphism of $\widetilde{A}\left[\overline{G_{\mathfrak{P}}}\right]$-modules:

$$
\widetilde{C}\left(\frac{\widetilde{O_{E}}}{\mathfrak{M} \widetilde{O_{E}}}\right) \simeq \frac{\widetilde{A}\left[\overline{G_{\mathfrak{P}}}\right]}{\left(P^{m} 1_{\overline{G_{\mathfrak{P}}}}-z^{d m \overline{\sigma_{\mathfrak{P}}}}\right) \widetilde{A}\left[\overline{G_{\mathfrak{P}}}\right]} .
$$

This implies that we have an isomorphism of $\widetilde{A}\left[G_{\mathfrak{P}}\right]$-modules:

$$
\widetilde{C}\left(\frac{\widetilde{O_{E}}}{\mathfrak{M} \widetilde{O_{E}}}\right) \simeq \frac{\widetilde{A}\left[G_{\mathfrak{P}}\right]}{\left(1_{G_{\mathfrak{P}}}+\left(P^{m}-1\right) u-z^{d m} \sigma_{\mathfrak{P}}\right) \widetilde{A}\left[G_{\mathfrak{P}}\right]},
$$

where $u=\frac{1}{\left|I_{\mathfrak{P}}\right|} \sum_{\delta \in I_{\mathfrak{P}}} \delta \in \mathbb{F}_{q}\left[I_{\mathfrak{P}}\right]$. Since, for $n \geq 1, \tau\left(\mathfrak{M}^{n}\right) \subset \mathfrak{M}^{n+1}$, we have:

$$
\left[\widetilde{C}\left(\frac{\mathfrak{M} \widetilde{O_{E}}}{\mathfrak{M}^{e} \widetilde{O_{E}}}\right)\right]_{\widetilde{A}\left[G_{\mathfrak{P}}\right]}=\left[\frac{\mathfrak{M} \widetilde{O_{E}}}{\mathfrak{M}^{e} \widetilde{O_{E}}}\right]_{\widetilde{A}\left[G_{\mathfrak{P}}\right]}
$$

Now, observe that:

$$
\left[\frac{\widetilde{O_{E}}}{\mathfrak{M O}_{E}}\right]_{\widetilde{A}\left[G_{\mathfrak{P}}\right]}=1_{G_{\mathfrak{P}}}+\left(P^{m}-1\right) u
$$


and:

Thus:

$$
\left[\frac{\mathfrak{M} \widetilde{O_{E}}}{\mathfrak{M}^{e} \widetilde{O_{E}}}\right]_{\widetilde{A}\left[G_{\mathfrak{P}}\right]}=\left(P^{m}-1\right)\left(1_{G_{\mathfrak{P}}}-u\right)+1_{G_{\mathfrak{P}}}
$$

$$
\left[\widetilde{C}\left(\frac{\widetilde{O_{E}}}{\mathfrak{M}^{e} \widetilde{O_{E}}}\right)\right]_{\widetilde{A}\left[G_{\mathfrak{P}}\right]}=P^{m} 1_{G_{\mathfrak{P}}}-z^{d m} \sigma_{\mathfrak{P}}
$$

But we have an isomorphism of $\widetilde{A}[G]$-modules:

$$
\widetilde{C}\left(\frac{\widetilde{O_{E}}}{\mathfrak{P} \widetilde{O_{E}}}\right) \simeq \widetilde{C}\left(\frac{\widetilde{O_{E}}}{\mathfrak{M}^{e} \widetilde{O_{E}}}\right) \otimes_{\mathbb{F}_{q}\left[G_{\mathfrak{P}}\right]} \mathbb{F}_{q}[G]
$$

We have:

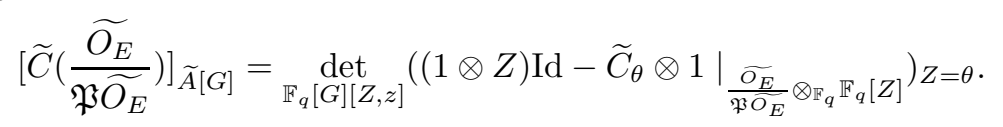

Thus:

$$
\left[\widetilde{C}\left(\frac{\widetilde{O_{E}}}{\mathfrak{P} \widetilde{O_{E}}}\right)\right]_{\widetilde{A}[G]}=\operatorname{det}_{\mathbb{F}_{q}\left[G_{\mathfrak{P}}\right][Z, z]}\left((1 \otimes Z) \operatorname{Id}-\widetilde{C}_{\theta} \otimes 1 \mid \frac{\widetilde{O_{E}}}{\frac{\mathfrak{M}^{e} O_{E}}{\mathbb{F}_{q}} \mathbb{F}_{q}[Z]}\right)_{Z=\theta} \otimes 1_{G}
$$

If $I$ is a non-zero ideal of $O_{L}$, recall that we have set:

$$
N_{L / K}(I)=\left[\frac{O_{L}}{I}\right]_{A}
$$

Write $I=\mathfrak{P}_{1}^{e_{1}} \cdots, \mathfrak{P}_{t}^{e_{t}}$ its decomposition into maximal ideals of $O_{L}$, we set:

$$
\sigma_{I}=\prod_{j=1}^{t} \sigma_{\mathfrak{P}_{j}}^{e_{j}} \in \mathbb{F}_{q}[G]
$$

By the above Lemma, we get:

$$
\mathcal{L}\left(\widetilde{C} /\left(\widetilde{O_{E}} / \widetilde{O_{L}}\right), G\right)=\sum_{I \text { ideal of } O_{L}, I \neq(0)} \frac{z^{\operatorname{deg}_{\theta} N_{L / K}(I)} \sigma_{I}}{N_{L / K}(I)} \in\left(T_{z}\left(K_{\infty}\right)[G]\right)^{\times} .
$$

Let $C$ be the Carlitz module, i.e. $C: A \rightarrow A\{\tau\}$ is the morphism of $\mathbb{F}_{q}$-algebras given by:

$$
C_{\theta}=\tau+\theta \text {. }
$$

Then Theorem 2.13 implies Anderson's log-algebraicity Theorem ([1], see also [5], [6]). More precisely we have:

Corollary 2.15. Let $L / K$ be a finite extension and let $m \geq 1$ be an integer. Let $x \in L_{\infty}$, and set:

$$
\ell_{m}(x)=\sum_{a \in A_{+}} \frac{C_{a}(x)^{m}}{a} z^{\operatorname{deg}_{\theta} a} \in L_{\infty}[[z]] .
$$

If $\ell_{m}(x)$ converges in $\mathbb{T}_{z}\left(L_{\infty}\right)$, then:

$$
\exp _{\widetilde{C}}\left(\ell_{m}(x)\right) \in A[z][x] \subset \mathbb{T}_{z}\left(L_{\infty}\right) .
$$

In that case:

$$
e v\left(\exp _{\widetilde{C}}\left(\ell_{m}(x)\right)\right)=\exp _{C}\left(e v\left(\ell_{m}(x)\right)\right) \in A[x] \subset L_{\infty}
$$


Proof. Let $P$ be a monic irreducible element in $A$ and let $M$ be the $P$ th cyclotomic function field ([25], chapter 12). Recall that $M / K$ is a finite abelian extension of degree $q^{d}-1$ where $d=\operatorname{deg}_{\theta} P$. Select $\lambda_{P} \in M^{\times}$such that $C_{P}\left(\lambda_{P}\right)=0$. Then (25, Proposition 12.9):

$$
O_{M}=A\left[\lambda_{P}\right]
$$

Furthermore, for $a \in A \backslash P A$, we have ([25], Theorem 12.10):

$$
\sigma_{a A}\left(\lambda_{P}\right)=C_{a}\left(\lambda_{P}\right)
$$

Also observe, that :

$$
\sigma_{P A}=-\sum_{g \in G} g,
$$

where $G=\operatorname{Gal}(M / K)$. For $a \in A_{+}$, we set:

$$
\sigma_{a}=\sigma_{a A}
$$

We get:

$$
\mathcal{L}\left(\widetilde{C} /\left(\widetilde{O_{M}} / \widetilde{A}\right), G\right)=\left(\sum_{a \in A_{+} \backslash P A_{+}} \frac{\sigma_{a} z^{\operatorname{deg}_{\theta} a}}{a}\right)\left(1+\frac{z^{d} \sum_{g \in G} g}{P}\right)^{-1} .
$$

Let $n \in\left\{1, \ldots, q^{d}-2\right\}$, then :

$$
\left(1+\frac{z^{d} \sum_{g \in G} g}{P}\right) \lambda_{P}^{n}=\lambda_{P}^{n} .
$$

Therefore, for $n \in\left\{1, \ldots, q^{d}-2\right\}$, by Theorem 2.13 we have:

$$
\mathcal{L}\left(\widetilde{C} /\left(\widetilde{O_{M}} / \widetilde{A}\right), G\right) \lambda_{P}^{n}=\sum_{a \in A_{+}} \frac{C_{a}\left(\lambda_{P}\right)^{n} z^{\operatorname{deg}_{\theta} a}}{a} \in U\left(\widetilde{C} / O_{M}[z]\right) .
$$

This implies that, for $n \in\left\{1, \ldots, q^{d}-2\right\}$, we have:

$$
\forall m \geq 0, \sum_{i+j=m} \frac{1}{D_{i}} \sum_{a \in A_{+, j}} \frac{\left(C_{a}\left(\lambda_{P}\right)^{n}\right)^{q^{i}}}{a^{q^{i}}} \in A\left[\lambda_{P}\right] .
$$

Now, let $X$ be an undeterminate over $K$. Let $\tau: K[X] \rightarrow K[X]$ be the morphism of $\mathbb{F}_{q^{-}}$-algebras such that $\forall x \in K[X], \tau(x)=x^{q}$. Let $n \geq 1$, and set:

$$
\forall m \geq 0, f_{m}(X)=\sum_{i+j=m} \frac{1}{D_{i}} \sum_{a \in A_{+, j}} \frac{\left(C_{a}(X)^{n}\right)^{q^{i}}}{a^{q^{i}}} \in K[X] .
$$

Fix an integer $m \geq 0$. Then, for all irreducible monic polynomials $P$ in $A$ :

$$
f_{m}\left(\lambda_{P}\right) \in A\left[\lambda_{P}\right] .
$$

This easily implies that:

We have:

$$
f_{m}(X) \in A[X] .
$$

$$
\exp _{\widetilde{C}}\left(\sum_{a \in A_{+}} \frac{C_{a}(X)^{n} z^{\operatorname{deg}_{\theta} a}}{a}\right)=\sum_{m \geq 0} f_{m}(X) z^{m} .
$$

Therefore, for all $n \geq 1$ :

$$
\exp _{\widetilde{C}}\left(\sum_{a \in A_{+}} \frac{C_{a}(X)^{n} z^{\operatorname{deg}_{\theta} a}}{a}\right) \in X^{n} A[X][[z]] .
$$


Now, we work in $\mathbb{T}_{z}\left(\mathbb{C}_{\infty}\right)$. Fix an integer $n \geq 1$. Then if $\lambda$ is a torsion point of the Carlitz module:

$$
\ell_{n}(\lambda) \in \mathbb{T}_{z}\left(\mathbb{C}_{\infty}\right)
$$

Observe that, for all $a \in A_{+}$, we have:

$$
v_{\infty}\left(C_{a}(\lambda)\right) \geq \frac{-1}{q-1} .
$$

Thus there exist $x_{\lambda} \in \mathbb{T}_{z}\left(\mathbb{C}_{\infty}\right)$ and $y(X) \in K[X][z]$ which does not depend on $\lambda$, such that :

$$
v_{\infty}\left(x_{\lambda}\right) \geq 1
$$

and:

$$
\exp _{\widetilde{C}}\left(\ell_{n}(\lambda)\right)=x_{\lambda}+\left.y\right|_{X=\lambda} .
$$

Thus, we have:

$$
\forall m \geq C(n), \forall \lambda, v_{\infty}\left(f_{m}(\lambda)\right) \geq 1,
$$

where $C(n)$ is a constant depending only on $n$. But, since for all $m \geq 0 f_{m}(X) \in$ $A[X]$, we get:

$$
\forall m \geq C(n), f_{m}(X)=0 .
$$

\section{Deformation of Drinfeld modules over several variable Tate ALGEBRAS}

The notion of deformation of a Drinfeld module over Tate algebras as introduced in section 3.1 has its roots in a remarkable formula obtained by F. Pellarin in 23. This formula links a certain one variable $L$-series to Anderson-Thakur special function. This formula was fully understood when F. Pellarin and the authors ([5]) found a connection with Taelman's work ([27]). In this section, we show how the ideas developed in section 2 can be extended to the situation of deformations of Drinfeld modules and we will study in details the case of the Carlitz module leading to the proof of the discrete Greenberg Conjectures. In the last section, we will construct $P$-adic $L$-series attached to deformations of Drinfeld modules.

\subsection{Basic properties.}

We fix some notation. Let $n \geq 0$ be a fixed integer and let $t_{1}, \ldots, t_{n}, z$ be $n+1$ indeterminates over $K_{\infty}$. We set $k=\mathbb{F}_{q}\left(t_{1}, \ldots, t_{n}\right), \mathbb{A}=k[\theta], \widetilde{\mathbb{A}}=k(z)[\theta]$, $\mathbb{K}_{\infty}=k\left(\left(\frac{1}{\theta}\right)\right), \widetilde{\mathbb{K}_{\infty}}=k(z)\left(\left(\frac{1}{\theta}\right)\right)$. We denote by $v_{\infty}$ the normalized $\frac{1}{\theta}$-adic valuation on $\widetilde{\mathbb{K}_{\infty}}$. Let $\mathbb{T}_{n, z}\left(K_{\infty}\right)$ be the closure of $K_{\infty}\left[t_{1}, \ldots, t_{n}, z\right]$ in $\widetilde{\mathbb{K}_{\infty}}$, and $\mathbb{T}_{n}\left(K_{\infty}\right)$ the closure of $K_{\infty}\left[t_{1}, \ldots, t_{n}\right]$ in $\mathbb{K}_{\infty}$. Let $\tau: \widetilde{\mathbb{K}_{\infty}} \rightarrow \widetilde{\mathbb{K}_{\infty}}$ be the continuous morphism of $k(z)$-algebras given by $\tau(\theta)=\theta^{q}$. Finally, we set $b_{0}(z)=1$, and for $m \geq 1$, $b_{m}(z)=\prod_{j=0}^{m-1}\left(z-\theta^{q^{j}}\right)$.

Let $\phi: A \rightarrow A\{\tau\}$ be a Drinfeld $A$-module defined over $A$, and write:

$$
\phi_{\theta}=\sum_{j=0}^{r} \alpha_{j} \tau^{j}, \alpha_{0}=\theta, \alpha_{j} \in A, \alpha_{r} \neq 0, r \geq 1
$$


Let $\varphi: \mathbb{A} \rightarrow \mathbb{A}\{\tau\}$ be the morphism of $k$-algebras given by:

$$
\varphi_{\theta}=\sum_{j=0}^{r} \alpha_{j} b_{j}\left(t_{1}\right) \cdots b_{j}\left(t_{n}\right) \tau^{j} .
$$

We call $\varphi$ the canonical deformation of $\phi$ over the Tate algebra $\mathbb{T}_{n}\left(K_{\infty}\right)$.

There exists a unique element $\exp _{\varphi} \in \mathbb{K}_{\infty}\{\{\tau\}\}$ such that $\exp _{\varphi} \equiv 1(\bmod \tau)$ and:

$$
\exp _{\varphi} \theta=\varphi_{\theta} \exp _{\varphi}
$$

If we write $\exp _{\phi}=\sum_{j \geq 0} e_{j} \tau^{j}$, we get:

$$
\exp _{\varphi}=\sum_{j \geq 0} b_{j}\left(t_{1}\right) \cdots b_{j}\left(t_{n}\right) e_{j} \tau^{j} .
$$

Observe that by 9 , Proposition 2.3 , $\exp _{\varphi}$ converges on $\mathbb{K}_{\infty}$ and furthermore $\exp _{\varphi}\left(\mathbb{T}_{n}\left(K_{\infty}\right)\right) \subset \mathbb{T}_{n}\left(K_{\infty}\right)$. Let's set:

$$
U(\varphi / \mathbb{A})=\left\{x \in \mathbb{K}_{\infty}, \exp _{\varphi}(x) \in \mathbb{A}\right\} .
$$

Then, by 9], Proposition 2.6, $U(\varphi / \mathbb{A})$ is a $\mathbb{A}$-lattice in $\mathbb{K}_{\infty}$, thus a free $\mathbb{A}$-module of rank one. Let's also set:

$$
H(\varphi / \mathbb{A})=\frac{\mathbb{K}_{\infty}}{\mathbb{A}+\exp _{\varphi}\left(\mathbb{K}_{\infty}\right)} .
$$

Then, by [9], Proposition 2.6, $H(\varphi / \mathbb{A})$ is a finite dimensional $k$-vector space and also a $\mathbb{A}$-module via $\varphi$. By [9], Theorem 2.7, the following infinite product converges in $\mathbb{K}_{\infty}$ :

$$
\mathcal{L}(\varphi / \mathbb{A}):=\prod_{P} \frac{\left[\frac{\mathbb{A}}{P \mathbb{A}}\right]_{\mathbb{A}}}{\left[\varphi\left(\frac{\mathbb{A}}{P \mathbb{A}}\right)\right]_{\mathbb{A}}} \in \mathbb{K}_{\infty}^{\times},
$$

where the product is over the monic irreducible polynomials in $A$. Since $\varphi_{\theta} \in$ $A\left[t_{1}, \ldots, t_{n}\right]\{\tau\}$, we deduce that in fact:

$$
\mathcal{L}(\varphi / \mathbb{A}) \in \mathbb{T}_{n}\left(K_{\infty}\right)^{\times} .
$$

We have the following result ( 9 , theorem 2.7):

$$
\mathcal{L}(\varphi / \mathbb{A}) \mathbb{A}=[H(\varphi / \mathbb{A})]_{\mathbb{A}} U(\varphi / \mathbb{A})
$$

Let's set:

$$
U\left(\varphi / A\left[t_{1}, \ldots, t_{n}\right]\right)=\left\{x \in \mathbb{T}_{n}\left(K_{\infty}\right), \exp _{\varphi}(x) \in A\left[t_{1}, \ldots, t_{n}\right]\right\} .
$$

Lemma 3.1. We have that $[H(\varphi / \mathbb{A})]_{\mathbb{A}} \in A\left[t_{1}, \ldots, t_{n}\right]$, and:

$$
[H(\varphi / \mathbb{A})]_{\mathbb{A}} U\left(\varphi / A\left[t_{1}, \ldots, t_{n}\right]\right)=\mathcal{L}(\varphi / \mathbb{A}) A\left[t_{1}, \ldots, t_{n}\right] .
$$

Proof. By a similar argument to that used in the proof of Proposition 2.3 we have that $U(\varphi / \mathbb{A})$ if equal to the $k$-vector space spanned by $U\left(\varphi / A\left[t_{1}, \ldots, t_{n}\right]\right)$. Recall that $\mathbb{T}_{n}\left(K_{\infty}\right)$ is a unique factorization domain ([12, chapter 3$)$. Now, select $\varepsilon \in U\left(\varphi / A\left[t_{1}, \ldots, t_{n}\right]\right)$ such that:

$$
U(\varphi / \mathbb{A})=\mathbb{A} \varepsilon .
$$

Observe that for $\delta \in \mathbb{F}_{q}\left[t_{1}, \ldots, t_{n}\right] \backslash\{0\}$, we have:

$$
A\left[t_{1}, \ldots, t_{n}\right] \cap \delta \mathbb{T}_{n}\left(K_{\infty}\right)=\delta A\left[t_{1}, \ldots, t_{n}\right] .
$$


This implies that we can assume that $\varepsilon$ is a primitive element in $\mathbb{T}_{n}\left(K_{\infty}\right)$, i.e. the elements of $\mathbb{F}_{q}\left[t_{1}, \ldots, t_{n}\right] \backslash\{0\}$ that divides $\varepsilon$ in $\mathbb{T}_{n}\left(K_{\infty}\right)$ are only the elements in $\mathbb{F}_{q}^{\times}$. Then by formula (5), there exists $x \in k^{\times}$such that:

$$
\mathcal{L}(\varphi / \mathbb{A})=[H(\varphi / \mathbb{A})]_{\mathbb{A}} x \varepsilon .
$$

But $\mathcal{L}(\varphi / \mathbb{A}) \in \mathbb{T}_{n}\left(K_{\infty}\right)^{\times}$and $\varepsilon$ is primitive, since $[H(\varphi / \mathbb{A})]_{\mathbb{A}}$ is monic, this implies that $[H(\varphi / \mathbb{A})]_{\mathbb{A}} \in A\left[t_{1}, \ldots, t_{n}\right], \varepsilon \in \mathbb{T}_{n}\left(K_{\infty}\right)^{\times}$, and:

$$
\mathcal{L}(\varphi / \mathbb{A})=\lambda[H(\varphi / \mathbb{A})]_{\mathbb{A}} \varepsilon
$$

for some $\lambda \in \mathbb{F}_{q}^{\times}$. Now let $m \in U\left(\varphi / A\left[t_{1}, \ldots, t_{n}\right]\right)$. By formula (5), there exists $x \in$ $\mathbb{F}_{q}\left[t_{1}, \ldots, t_{n}\right] \backslash\{0\}$ such that $x[H(\varphi / \mathbb{A})]_{\mathbb{A}} m \in A\left[t_{1}, \ldots, t_{n}\right] \mathcal{L}(\varphi / \mathbb{A})$. But $\mathcal{L}(\varphi / \mathbb{A}) \in$ $\mathbb{T}_{n}\left(K_{\infty}\right)^{\times}$and $\mathbb{T}_{n}\left(K_{\infty}\right)$ is a unique factorization domain, thus $[H(\varphi / \mathbb{A})]_{\mathbb{A}} m \in$ $A\left[t_{1}, \ldots, t_{n}\right] \mathcal{L}(\varphi / \mathbb{A})$.

Let's set:

$$
H\left(\varphi / A\left[t_{1}, \ldots, t_{n}\right]\right)=\frac{\mathbb{T}_{n}\left(K_{\infty}\right)}{A\left[t_{1}, \ldots, t_{n}\right]+\exp _{\varphi}\left(\mathbb{T}_{n}\left(K_{\infty}\right)\right.} .
$$

We observe that there exists an integer $N \geq 1$ such that $\exp _{\varphi}$ induces a bijective morphism of $\mathbb{F}_{q}\left[t_{1}, \ldots, t_{n}\right]$-modules on $\frac{1}{\theta^{N}} \mathbb{F}_{q}\left[t_{1}, \ldots, t_{n}\right]\left[\left[\frac{1}{\theta}\right]\right]$. Since we have $\mathbb{T}_{n}\left(K_{\infty}\right)=A\left[t_{1}, \ldots, t_{n}\right] \oplus \frac{1}{\theta} \mathbb{F}_{q}\left[t_{1}, \ldots, t_{n}\right]\left[\left[\frac{1}{\theta}\right]\right]$, we deduce that $H\left(\varphi / A\left[t_{1}, \ldots, t_{n}\right]\right)$ is a finitely generated $\mathbb{F}_{q}\left[t_{1}, \ldots, t_{n}\right]$-module and also an $A\left[t_{1}, \ldots, t_{n}\right]$-module via $\varphi$. Furthermore, the $k$-vector space spanned by $\mathbb{T}_{n}\left(K_{\infty}\right)$ is dense in $\mathbb{K}_{\infty}$, thus the natural inclusion $\mathbb{T}_{n}\left(K_{\infty}\right) \subset \mathbb{K}_{\infty}$ induces an isomorphism of $\mathbb{A}$-modules:

$$
H\left(\varphi / A\left[t_{1}, \ldots, t_{n}\right]\right) \otimes_{\mathbb{F}_{q}\left[t_{1}, \ldots, t_{n}\right]} k \simeq H(\varphi / \mathbb{A}) .
$$

However, note that the map $H\left(\varphi / A\left[t_{1}, \ldots, t_{n}\right]\right) \rightarrow H(\varphi / \mathbb{A})$ induced by the inclusion $\mathbb{T}_{n}\left(K_{\infty}\right) \subset \mathbb{K}_{\infty}$ is not injective in general.

Let $\widetilde{\varphi}$ be the canonical $z$-deformation of $\varphi$, i.e. : $\widetilde{\varphi}: \widetilde{\mathbb{A}} \rightarrow \widetilde{\mathbb{A}}\{\tau\}$ is the morphism of $k(z)$-algebras given by:

$$
\widetilde{\varphi}_{\theta}=\sum_{j=0}^{r} \alpha_{j} b_{j}\left(t_{1}\right) \cdots b_{j}\left(t_{n}\right) z^{j} \tau^{j} .
$$

There exists a unique element $\exp _{\widetilde{\varphi}} \in \widetilde{\mathbb{K}_{\infty}}\{\{\tau\}\}$ such that $\exp _{\widetilde{\varphi}} \equiv 1(\bmod \tau)$ and:

$$
\exp _{\widetilde{\varphi}} \theta=\widetilde{\varphi}_{\theta} \exp _{\varphi} \text {. }
$$

We have:

$$
\exp _{\widetilde{\varphi}}=\sum_{j \geq 0} b_{j}\left(t_{1}\right) \cdots b_{j}\left(t_{n}\right) e_{j} z^{j} \tau^{j}
$$

By [9], Proposition 2.3, $\exp _{\widetilde{\varphi}}$ converges on $\widetilde{\mathbb{K}_{\infty}}$ and furthermore $\exp _{\widetilde{\varphi}}\left(\mathbb{T}_{n, z}\left(K_{\infty}\right)\right) \subset$ $\mathbb{T}_{n, z}\left(K_{\infty}\right)$. Let's set:

$$
U(\widetilde{\varphi} / \widetilde{\mathbb{A}})=\left\{x \in \widetilde{\mathbb{K}_{\infty}}, \exp _{\widetilde{\varphi}}(x) \in \widetilde{\mathbb{A}}\right\}
$$

Then, by $\left[9\right.$, Proposition 2.6, $U(\widetilde{\varphi} / \widetilde{\mathbb{A}})$ is an $\widetilde{\mathbb{A}}$-lattice in $\widetilde{\mathbb{K}_{\infty}}$. Let's also set:

$$
H(\widetilde{\varphi} / \widetilde{\mathbb{A}})=\frac{\widetilde{\mathbb{K}_{\infty}}}{\widetilde{\mathbb{A}}+\exp _{\widetilde{\varphi}}\left(\widetilde{\mathbb{K}_{\infty}}\right)} .
$$


Then, by [9], Proposition 2.6, $H(\widetilde{\varphi} / \widetilde{\mathbb{A}})$ is a finite dimensional $k(z)$-vector space. By [9], Theorem 2.7, the following infinite product converges in $\widetilde{\mathbb{K}_{\infty}}$ :

$$
\mathcal{L}(\widetilde{\varphi} / \widetilde{\mathbb{A}}):=\prod_{P} \frac{\left[\frac{\widetilde{\mathbb{A}}}{P \widetilde{\mathbb{A}}}\right]_{\widetilde{\mathbb{A}}}}{\left[\widetilde{\varphi}\left(\frac{\widetilde{\mathbb{A}}}{P \widetilde{\mathbb{A}}}\right)\right]_{\widetilde{\mathbb{A}}}} \in{\widetilde{\mathbb{K}_{\infty}}}^{\times}
$$

where the product is over the monic irreducible polynomials in $A$. Since $\widetilde{\varphi}_{\theta} \in$ $A\left[t_{1}, \ldots, t_{n}, z\right]\{\tau\}$, we have:

$$
\mathcal{L}(\widetilde{\varphi} / \widetilde{\mathbb{A}}) \in \mathbb{T}_{n, z}\left(K_{\infty}\right)^{\times}
$$

We have the following formula $([9$, theorem 2.7):

$$
\mathcal{L}(\widetilde{\varphi} / \widetilde{\mathbb{A}}) \widetilde{\mathbb{A}}=[H(\widetilde{\varphi} / \widetilde{\mathbb{A}})]_{\widetilde{\mathbb{A}}} U(\widetilde{\varphi} / \widetilde{\mathbb{A}})
$$

Let's set:

$$
U\left(\widetilde{\varphi} / A\left[t_{1}, \ldots, t_{n}, z\right]\right)=\left\{x \in \mathbb{T}_{n, z}\left(K_{\infty}\right), \exp _{\widetilde{\varphi}}(x) \in A\left[t_{1}, \ldots, t_{n}, z\right]\right\},
$$

and:

$$
H\left(\tilde{\varphi} / A\left[t_{1}, \ldots, t_{n}, z\right]\right)=\frac{\mathbb{T}_{n, z}\left(K_{\infty}\right)}{A\left[t_{1}, \ldots, t_{n}, z\right]+\exp _{\widetilde{\varphi}}\left(\mathbb{T}_{n, z}\left(K_{\infty}\right)\right)} .
$$

As for $\varphi, H\left(\widetilde{\varphi} / A\left[t_{1}, \ldots, t_{n} z\right]\right)$ is a finitely generated $\mathbb{F}_{q}\left[t_{1}, \ldots, t_{n}, z\right]$-module and the inclusion $\mathbb{T}_{n, z}\left(K_{\infty}\right) \subset \widetilde{\mathbb{K}_{\infty}}$ induces an isomorphism of $\widetilde{A}$-modules:

$$
H\left(\widetilde{\varphi} / A\left[t_{1}, \ldots, t_{n} z\right]\right) \otimes_{\mathbb{F}_{q}\left[t_{1}, \ldots, t_{n}, z\right]} k(z) \simeq H(\widetilde{\varphi} / \widetilde{\mathbb{A}}) .
$$

Proposition 3.2. We have:

$$
H(\widetilde{\varphi} / \widetilde{\mathbb{A}})=\{0\}
$$

Furthermore:

$$
U\left(\widetilde{\varphi} / A\left[t_{1}, \ldots, t_{n}, z\right]\right)=\mathcal{L}(\widetilde{\varphi} / \widetilde{\mathbb{A}}) A\left[t_{1}, \ldots, t_{n}, z\right] .
$$

Proof. The proof of the first assertion is similar to that of Proposition 2.4. For the convenience of the reader, we give its proof.

We have:

$$
\mathbb{T}_{n, z}\left(K_{\infty}\right)=\mathbb{T}_{n}\left(K_{\infty}\right) \oplus z \mathbb{T}_{n, z}\left(K_{\infty}\right)
$$

Since $\forall x \in \mathbb{T}_{n, z}\left(K_{\infty}\right),\left.\exp _{\widetilde{\varphi}}(x) \equiv x\right|_{z=0}\left(\bmod z \mathbb{T}_{n, z}\left(K_{\infty}\right)\right)$, we deduce that the multiplication by $z$ gives rise to an exact sequence of $A\left[t_{1}, \ldots, t_{n}, z\right]$-modules:

$$
0 \rightarrow M \rightarrow H\left(\widetilde{\varphi} / A\left[t_{1}, \ldots, t_{n}, z\right]\right) \rightarrow H\left(\widetilde{\varphi} / A\left[t_{1}, \ldots, t_{n}, z\right]\right) \rightarrow 0
$$

where $M=\left\{x \in H\left(\widetilde{\varphi} / A\left[t_{1}, \ldots, t_{n}, z\right]\right), z x=0\right\}$.

This implies that $H\left(\widetilde{\varphi} / A\left[t_{1}, \ldots, t_{n}, z\right]\right) \otimes_{\mathbb{F}_{q}\left[t_{1}, \ldots, t_{n}\right]} k$ is a finitely generated and torsion $k[z]$-module. Thus we get the desired assertion.

We deduce the second assertion by formula (7) and a similar proof to that of Lemma 3.1

We set:

$$
u_{\phi}\left(t_{1}, \ldots, t_{n} ; z\right)=\exp _{\widetilde{\varphi}}(\mathcal{L}(\widetilde{\varphi} / \widetilde{\mathbb{A}})) \in A\left[t_{1}, \ldots, t_{n}, z\right] .
$$

Since $\exp _{\tilde{\phi}}: \mathbb{T}_{n, z}\left(K_{\infty}\right) \rightarrow \mathbb{T}_{n, z}\left(K_{\infty}\right)$ is an injective morphism of $\mathbb{F}_{q}\left[t_{1}, \ldots, t_{n}, z\right]$ modules, we have that:

$$
u_{\phi}\left(t_{1}, \ldots, t_{n} ; z\right) \neq 0 \text {. }
$$


Remark 3.3. Select $\tilde{\pi}$ a period of the Carlitz module (well-defined modulo $\mathbb{F}_{q}^{\times}$). Let $h \in\{0, \ldots, q-2\}$ such that $h \equiv n(\bmod q-1)$. Observe that $\widetilde{\pi}^{h} K_{\infty}$ is an $A$-module via $\phi$ and that $\exp _{\phi}\left(\widetilde{\pi}^{h} K_{\infty}\right) \subset \widetilde{\pi}^{h} K_{\infty}$. Therefore if $\exp _{\phi}$ is not injective on $\widetilde{\pi}^{h} K_{\infty}$, there exists $\pi_{\phi, h} \in \widetilde{\pi}^{h} K_{\infty} \backslash\{0\}$ such that:

$$
\operatorname{Ker}\left(\exp _{\phi}: \widetilde{\pi}^{h} K_{\infty} \rightarrow \widetilde{\pi}^{h} K_{\infty}\right)=A \pi_{\phi, h}
$$

Now observe that:

$$
\frac{\omega\left(t_{1}\right) \cdots \omega\left(t_{n}\right)}{\widetilde{\pi}^{h}} \in \mathbb{T}_{n}\left(K_{\infty}\right)
$$

where for $j=1, \ldots, n$, we have set:

$$
\omega\left(t_{j}\right)=\exp _{C}\left(\frac{\widetilde{\pi}}{\theta}\right) \prod_{k \geq 0}\left(1-\frac{t_{j}}{\theta^{q^{k}}}\right)^{-1} \in \widetilde{\pi} \mathbb{T}_{n}\left(K_{\infty}\right)^{\times} .
$$

Furthermore, for $f \in \mathbb{T}_{n}\left(K_{\infty}\right)$, we have:

$$
\omega\left(t_{1}\right) \cdots \omega\left(t_{n}\right) \exp _{\varphi}(f)=\exp _{\phi}\left(\omega\left(t_{1}\right) \cdots \omega\left(t_{n}\right) f\right) .
$$

Therefore, $\exp _{\varphi}$ is injective on $\mathbb{T}_{n}\left(K_{\infty}\right)$ if and only if $\exp _{\phi}$ is injective on $\widetilde{\pi}^{h} K_{\infty}$. Otherwise :

$$
\operatorname{Ker}\left(\exp _{\varphi}: \mathbb{T}_{n}\left(K_{\infty}\right) \rightarrow \mathbb{T}_{n}\left(K_{\infty}\right)\right)=A\left[t_{1}, \ldots, t_{n}\right] \frac{\pi_{\phi, h}}{\omega\left(t_{1}\right) \cdots \omega\left(t_{n}\right)} .
$$

We assume now that $\exp _{\varphi}$ is not injective on $\mathbb{T}_{n}\left(K_{\infty}\right)$. Observe that:

$$
\frac{\pi_{\phi, h}}{\omega\left(t_{1}\right) \cdots \omega\left(t_{n}\right)} \in \mathbb{T}_{n}\left(K_{\infty}\right)^{\times} .
$$

By similar arguments than those used in the proof of Lemma 3.1, we deduce that there exists $a \in A\left[t_{1}, \ldots, t_{n}\right]$ which is monic as a polynomial in $\theta$ such that:

$$
U\left(\varphi / A\left[t_{1}, \ldots, t_{n}\right]\right)=A\left[t_{1}, \ldots, t_{n}\right] \frac{\pi_{\phi, h}}{a \omega\left(t_{1}\right) \cdots \omega\left(t_{n}\right)} .
$$

Therefore, there exists $\lambda \in \mathbb{F}_{q}^{\times}$such that:

$$
\frac{\mathcal{L}(\varphi / \mathbb{A})}{[H(\varphi / \mathbb{A})]_{\mathbb{A}}}=\lambda \frac{\pi_{\phi, h}}{a \omega\left(t_{1}\right) \cdots \omega\left(t_{n}\right)} .
$$

\subsection{Evaluation at $z=1$.}

We keep the hypothesis and notation of section 3.1. Let's consider the continuous morphism of $\mathbb{F}_{q}\left[t_{1}, \ldots, t_{n}\right]$-algebras $e v: \mathbb{T}_{n, z}\left(K_{\infty}\right) \rightarrow \mathbb{T}_{n}\left(K_{\infty}\right)$ given by:

$$
\forall f \in \mathbb{T}_{n, z}\left(K_{\infty}\right), e v(f)=\left.f\right|_{z=1} .
$$

Then:

$$
\text { Ker } e v=(z-1) \mathbb{T}_{n, z}\left(K_{\infty}\right)
$$

Furthermore, for $f \in \mathbb{T}_{n, z}\left(K_{\infty}\right)$, we have:

$$
\begin{aligned}
e v\left(\exp _{\widetilde{\varphi}}(f)\right) & =\exp _{\varphi}(e v(f)), \\
e v\left(\widetilde{\varphi}_{\theta}(f)\right) & =\varphi_{\theta}(e v(f)) .
\end{aligned}
$$

This implies that $e v$ gives rise to an isomorphism of $A\left[t_{1}, \ldots, t_{n}\right]$-modules:

$$
\frac{H\left(\widetilde{\varphi} / A\left[t_{1}, \ldots, t_{n}, z\right]\right)}{(z-1) H\left(\widetilde{\varphi} / A\left[t_{1}, \ldots, t_{n}, z\right]\right)} \simeq H\left(\varphi / A\left[t_{1}, \ldots, t_{n}\right]\right)
$$

Let's set:

$$
U_{S t}\left(\varphi / A\left[t_{1}, \ldots, t_{n}\right]\right)=e v\left(U\left(\widetilde{\varphi} / A\left[t_{1}, \ldots, t_{n}, z\right]\right)\right) .
$$


Clearly $U_{S t}\left(\varphi / A\left[t_{1}, \ldots, t_{n}\right]\right)$ is a sub- $A\left[t_{1}, \ldots, t_{n}\right]$-module of $U\left(\varphi / A\left[t_{1}, \ldots, t_{n}\right]\right)$, and by Proposition 3.2, we have:

$$
U_{S t}\left(\varphi / A\left[t_{1}, \ldots, t_{n}\right]\right)=\mathcal{L}(\varphi / \mathbb{A}) A\left[t_{1}, \ldots, t_{n}\right] .
$$

In particular, by Lemma 3.1, we have an isomorphism of $A\left[t_{1}, \ldots, t_{n}\right]$-modules:

$$
\frac{U\left(\varphi / A\left[t_{1}, \ldots, t_{n}\right]\right)}{U_{S t}\left(\varphi / A\left[t_{1}, \ldots, t_{n}\right]\right)} \simeq \frac{A\left[t_{1}, \ldots, t_{n}\right]}{[H(\varphi / \mathbb{A})]_{\mathbb{A}} A\left[t_{1}, \ldots, t_{n}\right]} .
$$

Let $\alpha: \mathbb{T}_{n, z}\left(K_{\infty}\right) \rightarrow \mathbb{T}_{n z}\left(K_{\infty}\right)$ be the morphism of $\mathbb{F}_{q}\left[t_{1}, \ldots, t_{n}, z\right]$-modules given by:

$$
\forall f \in \mathbb{T}_{n, z}\left(K_{\infty}\right), \alpha(f)=\frac{\exp _{\widetilde{\varphi}}(f)-\exp _{\varphi}(f)}{z-1}
$$

Let's set:

$$
H\left(\widetilde{\varphi} / A\left[t_{1}, \ldots, t_{n}, z\right]\right)[z-1]=\left\{x \in H\left(\widetilde{\varphi} / A\left[t_{1}, \ldots, t_{n}, z\right]\right),(z-1) x=0\right\} .
$$

Observe that $H\left(\widetilde{\varphi} / A\left[t_{1}, \ldots, t_{n}, z\right]\right)[z-1]$ is an $A\left[t_{1}, \ldots, t_{n}\right]$-module via $\widetilde{\varphi}$.

Proposition 3.4. The map $\alpha$ induces an isomorphism of $A\left[t_{1}, \ldots, t_{n}\right]$-modules:

$$
\frac{U\left(\varphi / A\left[t_{1}, \ldots, t_{n}\right]\right)}{U_{S t}\left(\varphi / A\left[t_{1}, \ldots, t_{n}\right]\right)} \simeq H\left(\widetilde{\varphi} / A\left[t_{1}, \ldots, t_{n}, z\right]\right)[z-1] .
$$

Proof. The above result can be proved along the same lines as that of the proof of Proposition 2.6. For the convenience of the reader, we give a proof. The map $\exp _{\tilde{\varphi}}$ induces the exact sequence of $A\left[t_{1}, \ldots, t_{n}, z\right]$-modules :

$$
0 \rightarrow M_{1} \rightarrow M_{2} \rightarrow M_{3} \rightarrow 0
$$

where

$$
M_{1}:=\frac{\mathbb{T}_{n, z}\left(K_{\infty}\right)}{U\left(\widetilde{\varphi} / A\left[t_{1}, \ldots, t_{n}, z\right]\right)} ; M_{2}:=\frac{\mathbb{T}_{n, z}\left(K_{\infty}\right)}{A\left[t_{1}, \ldots, t_{n}, z\right]} ; M_{3}:=H\left(\widetilde{\varphi} / A\left[t_{1}, \ldots, t_{n}, z\right]\right) .
$$

Thus, by the snake Lemma, we get a long exact sequence:

$$
\begin{aligned}
& 0 \longrightarrow M_{1}[z-1] \longrightarrow M_{2}[z-1] \longrightarrow M_{3}[z-1] \longrightarrow \\
\longrightarrow & \frac{M_{1}}{(z-1) M_{1}} \longrightarrow \frac{M_{2}}{(z-1) M_{2}} \longrightarrow \frac{M_{3}}{(z-1) M_{3}} \longrightarrow 0 .
\end{aligned}
$$

Now, we observe that the map $e v$ induces isomorphisms of $A\left[t_{1}, \ldots, t_{n}\right]$-modules:

$$
\begin{gathered}
\frac{M_{1}}{(z-1) M_{1}} \simeq \frac{\mathbb{T}_{n}\left(K_{\infty}\right)}{U_{S t}\left(\varphi / A\left[t_{1}, \ldots, t_{n}\right]\right)}, \\
\frac{M_{2}}{(z-1) M_{2}} \simeq \frac{\mathbb{T}_{n}\left(K_{\infty}\right)}{A\left[t_{1}, \ldots, t_{n}\right]}, \\
\frac{M_{3}}{(z-1) M_{3}} \simeq H\left(\varphi / A\left[t_{1}, \ldots, t_{n}\right]\right) .
\end{gathered}
$$

Observe also that:

$$
(z-1) \mathbb{T}_{n, z}\left(K_{\infty}\right) \cap A\left[t_{1}, \ldots, t_{n}, z\right]=(z-1) A\left[t_{1}, \ldots, t_{n}, z\right] .
$$

Therefore:

$$
M_{2}[z-1]=\{0\}
$$


Let $\delta: M_{3}[z-1] \rightarrow \frac{M_{1}}{(z-1) M_{1}}$ be the map given by the snake Lemma. Let $x \in M_{3}[z-$ $1]$ and select $y \in \mathbb{T}_{n, z}\left(K_{\infty}\right)$ such that $x \equiv y\left(\bmod A\left[t_{1}, \ldots, t_{n}, z\right]+\exp _{\widetilde{\varphi}}\left(\mathbb{T}_{n, z}\left(K_{\infty}\right)\right)\right.$. Then:

$$
(z-1) y=\exp _{\widetilde{\varphi}}(m)+h
$$

where $m \in \mathbb{T}_{n, z}\left(K_{\infty}\right)$ and $h \in A\left[t_{1}, \ldots, t_{n}, z\right]$. We get:

$$
\delta(x)=m \quad\left(\bmod (z-1) \mathbb{T}_{n, z}\left(K_{\infty}\right)+U\left(\widetilde{\varphi} / A\left[t_{1}, \ldots, t_{n}, z\right]\right)\right) .
$$

But we have:

$$
e v((z-1) y)=\exp _{\varphi}(e v(m))+e v(h)=0
$$

Thus $\beta=e v(m) \in U\left(\varphi / A\left[t_{1}, \ldots, t_{n}\right]\right)$. Thus:

$$
\delta(x) \equiv \beta \quad\left(\bmod (z-1) \mathbb{T}_{n, z}\left(K_{\infty}\right)+U\left(\widetilde{\varphi} / A\left[t_{1}, \ldots, t_{n}, z\right]\right)\right) .
$$

Furthermore:

$(z-1) y \equiv \exp _{\widetilde{\varphi}}(\beta)-\exp _{\varphi}(\beta) \quad\left(\bmod (z-1) \exp _{\widetilde{\varphi}}\left(\mathbb{T}_{n, z}\left(K_{\infty}\right)\right)+(z-1) A\left[t_{1}, \ldots, t_{n}, z\right]\right)$, and therefore:

$$
\alpha(\beta) \equiv x \quad\left(\bmod \exp _{\widetilde{\varphi}}\left(\mathbb{T}_{n, z}\left(K_{\infty}\right)\right)+A\left[t_{1}, \ldots, t_{n}, z\right]\right) .
$$

Therefore, we get an exact sequence of $A\left[t_{1}, \ldots, t_{n}\right]$-modules:

$$
\begin{aligned}
0 \longrightarrow H\left(\widetilde{\varphi} / A\left[t_{1}, \ldots, t_{n}, z\right]\right)[z-1] & \longrightarrow \frac{\mathbb{T}_{n}\left(K_{\infty}\right)}{U_{S t}\left(\varphi / A\left[t_{1}, \ldots, t_{n}\right]\right)} \longrightarrow \\
\longrightarrow \frac{\mathbb{T}_{n}\left(K_{\infty}\right)}{A\left[t_{1}, \ldots, t_{n}\right]} & \longrightarrow H\left(\varphi / A\left[t_{1}, \ldots, t_{n}\right]\right) \longrightarrow 0
\end{aligned}
$$

where the exact sequence $\frac{\mathbb{T}_{n}\left(K_{\infty}\right)}{U_{S t}\left(\varphi / A\left[t_{1}, \ldots, t_{n}\right]\right)} \rightarrow \frac{\mathbb{T}_{n}\left(K_{\infty}\right)}{A\left[t_{1}, \ldots, t_{n}\right]} \rightarrow H\left(\varphi / A\left[t_{1}, \ldots, t_{n}\right]\right) \rightarrow 0$ is induced by $\exp _{\varphi}$, and where the map $H\left(\widetilde{\varphi} / A\left[t_{1}, \ldots, t_{n}, z\right]\right)[z-1] \rightarrow \frac{\mathbb{T}_{n}\left(K_{\infty}\right)}{U_{S t}\left(\varphi / A\left[t_{1}, \ldots, t_{n}\right]\right)}$ sends $x \in H\left(\widetilde{\varphi} / A\left[t_{1}, \ldots, t_{n}, z\right]\right)[z-1]$ to $\beta$ for some $\beta \in U\left(\varphi / A\left[t_{1}, \ldots, t_{n}\right]\right)$ such that $\alpha(\beta) \equiv x\left(\bmod \mathbb{T}_{n, z}\left(K_{\infty}\right)+A\left[t_{1}, \ldots, t_{n}, z\right]\right)$.

Let $\frac{d}{d z}: \mathbb{T}_{n, z}\left(K_{\infty}\right)\{\{\tau\}\} \rightarrow \mathbb{T}_{n, z}\left(K_{\infty}\right)\{\{\tau\}\}$ be the map defined by:

$$
\frac{d}{d z}\left(\sum_{i \geq 0} f_{i} \tau^{i}\right)=\sum_{i \geq 0} \frac{d}{d z}\left(f_{i}\right) \tau^{i}, f_{i} \in \mathbb{T}_{n, z}
$$

where $\frac{d}{d z}\left(\sum_{i \geq 0} x_{i} z^{i}\right)=\sum_{i \geq 1} i x_{i} z^{i-1}, x_{i} \in \mathbb{T}_{n}\left(K_{\infty}\right)$. We set:

$$
\exp _{\varphi}^{(1)}=\sum_{j \geq 1} j b_{j}\left(t_{1}\right) \cdots b_{j}\left(t_{n}\right) e_{j} \tau^{j}
$$

Corollary 3.5. The map $\exp _{\varphi}^{(1)}$ induces a morphism of $A\left[t_{1}, \ldots, t_{n}\right]$-modules:

$$
\frac{U\left(\varphi / A\left[t_{1}, \ldots, t_{n}\right]\right)}{U_{S t}\left(\varphi / A\left[t_{1}, \ldots, t_{n}\right]\right)} \rightarrow H\left(\varphi / A\left[t_{1}, \ldots, t_{n}\right]\right) \text {. }
$$

Let $H^{(1)}\left(\varphi / A\left[t_{1}, \ldots, t_{n}\right]\right)$ be the image of the above map. The kernel of the above map is isomorphic as an $A\left[t_{1}, \ldots, t_{n}\right]$-module to:

$$
\frac{H\left(\widetilde{\varphi} / A\left[t_{1}, \ldots, t_{n}, z\right]\right)\left[(z-1)^{2}\right]}{H\left(\widetilde{\varphi} / A\left[t_{1}, \ldots, t_{n}, z\right]\right)[z-1]}
$$


where $H\left(\widetilde{\varphi} / A\left[t_{1}, \ldots, t_{n}, z\right]\right)\left[(z-1)^{2}\right]=\left\{x \in H\left(\widetilde{\varphi} / A\left[t_{1}, \ldots, t_{n}, z\right]\right),(z-1)^{2} x=0\right\}$. In particular if $H\left(\tilde{\varphi} / A\left[t_{1}, \ldots, t_{n}, z\right]\right)\left[(z-1)^{2}\right]=H\left(\widetilde{\varphi} / A\left[t_{1}, \ldots, t_{n}, z\right]\right)[z-1]$, then we have an isomorphism of $A\left[t_{1}, \ldots, t_{n}\right]$-modules:

$$
H^{(1)}\left(\varphi / A\left[t_{1}, \ldots, t_{n}\right]\right) \simeq \frac{A\left[t_{1}, \ldots, t_{n}\right]}{[H(\varphi / \mathbb{A})]_{\mathbb{A}} A\left[t_{1}, \ldots, t_{n}\right]} .
$$

Furthermore, in this case, $\frac{H\left(\varphi / A\left[t_{1}, \ldots, t_{n}\right]\right)}{H^{(1)}\left(\varphi / A\left[t_{1}, \ldots, t_{n}\right]\right)}$ is a finitely generated and torsion $\mathbb{F}_{q}\left[t_{1}, \ldots, t_{n}\right]$-module.

Proof. Observe that $e v$ induces a morphism of $A\left[t_{1}, \ldots, t_{n}\right]$-modules:

$$
H\left(\widetilde{\varphi} / A\left[t_{1}, \ldots, t_{n}, z\right]\right)[z-1] \rightarrow H\left(\varphi / A\left[t_{1}, \ldots, t_{n}\right]\right) .
$$

The kernel of the above map being:

$$
(z-1) H\left(\widetilde{\varphi} / A\left[t_{1}, \ldots, t_{n}, z\right]\right)\left[(z-1)^{2}\right] .
$$

Now observe that, by formula (9), we have :

$$
\forall \beta \in U\left(\varphi / A\left[t_{1}, \ldots, t_{n}\right]\right), e v \circ \alpha(\beta)=\exp _{\varphi}^{(1)}(\beta) .
$$

It remains to apply Proposition 3.4 to obtain the first three assertions. The last assertion is a direct consequence of (6)

Question 3.6. Let $n \geq 1$.

1) Is it true that $H\left(\widetilde{\varphi} / A\left[t_{1}, \ldots, t_{n}, z\right]\right)\left[(z-1)^{2}\right]=H\left(\widetilde{\varphi} / A\left[t_{1}, \ldots, t_{n}, z\right]\right)[z-1]$ ?

2) Is $H(\varphi / \mathbb{A})$ a cyclic $\mathbb{A}$-module?

Observe that by the above Corollary if 1) is true then 2) is true. Also observe that 1) has a positive answer if and only if $\frac{H\left(\varphi / A\left[t_{1}, \ldots, t_{n}\right]\right)}{H^{(1)}\left(\varphi / A\left[t_{1}, \ldots, t_{n}\right]\right)}$ is a finitely generated and torsion $\mathbb{F}_{q}\left[t_{1}, \ldots, t_{n}\right]$-module. We are going to show in the next section that 1$)$ is true when $\phi$ is the Carlitz module.

\subsection{The case of the Carlitz module.}

In this paragraph $\phi=C$ is the Carlitz module, $\varphi$ is the canonical deformation of $C$ over $\mathbb{T}_{n}\left(K_{\infty}\right)$, and $\widetilde{\varphi}$ is the canonical $z$-deformation of $\varphi$.

3.3.1. Some results on units.

Observe that in our case (by [5], Proposition 5.9):

$$
\mathcal{L}(\widetilde{\varphi} / \widetilde{\mathbb{A}})=\sum_{a \in A_{+}} \frac{a\left(t_{1}\right) \cdots a\left(t_{n}\right)}{a} z^{\operatorname{deg}_{\theta} a} \in \mathbb{T}_{n, z}\left(K_{\infty}\right)^{\times},
$$

and:

$$
\mathcal{L}(\varphi / \mathbb{A})=\operatorname{ev}(\mathcal{L}(\widetilde{\varphi} / \mathbb{A}))=\sum_{a \in A_{+}} \frac{a\left(t_{1}\right) \cdots a\left(t_{n}\right)}{a} \in \mathbb{T}_{n}\left(K_{\infty}\right)^{\times} .
$$

Recall that we have set:

$$
u_{C}\left(t_{1}, \ldots, t_{n} ; z\right):=\exp _{\widetilde{\varphi}}(\mathcal{L}(\widetilde{\varphi} / \widetilde{\mathbb{A}})) \in A\left[t_{1}, \ldots, t_{n}, z\right] .
$$

Lemma 3.7. If $n \in\{0, \ldots, q-1\}$, then:

$$
u_{C}\left(t_{1}, \ldots, t_{n} ; z\right)=1 .
$$


Proof. Note that:

$$
\forall j \geq 0, v_{\infty}\left(\frac{b_{j}\left(t_{1}\right) \cdots b_{j}\left(t_{n}\right)}{D_{j}} z^{j}\right)=j q^{j}-n \frac{q^{j}-1}{q-1},
$$

and

$$
v_{\infty}(\mathcal{L}(\widetilde{\varphi} / \widetilde{\mathbb{A}})-1) \geq 1
$$

Thus, under the assumption of the Lemma, a direct computation gives:

$$
v_{\infty}\left(\exp _{\widetilde{\varphi}}(\mathcal{L}(\widetilde{\varphi} / \widetilde{\mathbb{A}}))-1\right) \geq 1 .
$$

Since $u_{C}\left(t_{1}, \ldots, t_{n} ; z\right) \in A\left[t_{1}, \ldots, t_{n}, z\right]$, we get the desired result.

Proposition 3.8. Let $n \geq q$. Write $n=q+r+\ell(q-1), \ell \in \mathbb{N}, r \in\{0, \ldots, q-$ $2\}$. Then $u_{C}\left(t_{1}, \ldots, t_{n} ; z\right)$ viewed as a polynomial in $\theta$ is of degree $n\left(\frac{q^{\ell}-1}{q-1}\right)-\ell q^{\ell}$ if $r=0$ and $n\left(\frac{q^{\ell+1}-1}{q-1}\right)-(\ell+1) q^{\ell+1}$ if $r \neq 0$. Furthermore the leading coefficient of $u_{C}\left(t_{1}, \ldots, t_{n} ; z\right)$ viewed as a polynomial in $\theta$ is :

$$
\begin{aligned}
& \text { if } r=0,(-1)^{\ell} z^{\ell}(1-z) . \\
& \text { if } r \neq 0,(-1)^{n(\ell+1)} z^{\ell+1} .
\end{aligned}
$$

Proof. We prove the result for $r=0$, the proof of the Proposition being similar in the remaining cases. For $i \geq 0$, observe that $\tau^{i}(\mathcal{L}(\widetilde{\varphi} / \widetilde{\mathbb{A}}))$ and $(-1)^{i} \frac{b_{i}\left(t_{1}\right) \cdots b_{i}\left(t_{n}\right)}{D_{i}}$ are monic. We have:

$$
\forall i \geq 0, v_{\infty}\left(\frac{b_{i}\left(t_{1}\right) \cdots b_{i}\left(t_{n}\right) z^{i}}{D_{i}} \tau^{i}(\mathcal{L}(\widetilde{\varphi} / \widetilde{\mathbb{A}}))\right)=i q^{i}-n\left(\frac{q^{i}-1}{q-1}\right) .
$$

Set, for $i \geq 0, \alpha_{i}=(-1)^{i} \frac{b_{i}\left(t_{1}\right) \cdots b_{i}\left(t_{n}\right)}{D_{i}} \tau^{i}(\mathcal{L}(\widetilde{\varphi} / \widetilde{\mathbb{A}}))$, then $\alpha_{i}$ is monic. We have:

$$
u\left(t_{1}, \ldots, t_{n} ; z\right)=\sum_{i \geq 0}(-1)^{i} \alpha_{i} z^{i} .
$$

For $i \geq 0$, we have :

$$
\left((i+1) q^{i+1}-n\left(\frac{q^{i+1}-1}{q-1}\right)\right)-\left(i q^{i}-n\left(\frac{q^{i}-1}{q-1}\right)\right)=q^{i}(q+i(q-1)-n) .
$$

Recall that $n=q+\ell(q-1), \ell \in \mathbb{N}$. We get :

$$
v_{\infty}\left(u_{C}\left(t_{1}, \ldots, t_{n} ; z\right)-\left((-1)^{\ell} \alpha_{\ell} z^{\ell}+(-1)^{(\ell+1)} \alpha_{\ell+1} z^{\ell+1}\right)\right)>v_{\infty}\left(u\left(t_{1}, \ldots, t_{n} ; z\right)\right) .
$$

But $\alpha_{\ell}$ and $\alpha_{\ell+1}$ are monic, thus:

$$
v_{\infty}\left(u_{C}\left(t_{1}, \ldots, t_{n} ; z\right)-\left(z^{\ell}(1-z)(-1)^{\ell} \theta^{n\left(\frac{q^{\ell}-1}{q-1}\right)-\ell q^{\ell}}\right)>v_{\infty}\left(u\left(t_{1}, \ldots, t_{n} ; z\right)\right) .\right.
$$

This proves the assertion.

Lemma 3.9. Let $n \geq q, n \equiv 1(\bmod q-1)$. Then:

$$
u_{C}\left(t_{1}, \ldots, t_{n} ; z\right) \in(z-1) A\left[t_{1}, \ldots, t_{n}, z\right] .
$$

Proof. By [5], Lemma 6.8 and Remark 3.3 exp $\exp _{\varphi}: \mathbb{T}_{n}\left(K_{\infty}\right) \rightarrow \mathbb{T}_{n}\left(K_{\infty}\right)$ is not injective if and only if $n \equiv 1(\bmod q-1)$. If $n \equiv 1(\bmod q-1)$, then:

$$
\operatorname{Ker}\left(\exp _{\varphi}: \mathbb{T}_{n}\left(K_{\infty}\right) \rightarrow \mathbb{T}_{n}\left(K_{\infty}\right)\right)=A\left[t_{1}, \ldots, t_{n}\right] \frac{\tilde{\pi}}{\omega\left(t_{1}\right) \cdots \omega\left(t_{n}\right)} .
$$


Furthermore, if $n \geq q, n \equiv 1(\bmod q-1)$, we have $([\underline{5}$, Proposition 7.2):

(12) $U\left(\varphi / A\left[t_{1}, \ldots, t_{n}\right]\right)=\left.\operatorname{Ker} \exp _{\varphi}\right|_{\mathbb{T}_{n}\left(K_{\infty}\right)}=A\left[t_{1}, \ldots, t_{n}\right] \frac{\tilde{\pi}}{\omega\left(t_{1}\right) \cdots \omega\left(t_{n}\right)}$.

Recall that $\mathcal{L}(\varphi / \mathbb{A}) A\left[t_{1}, \ldots, t_{n}\right]=U_{S t}\left(\varphi / A\left[t_{1}, \ldots, t_{n}\right]\right) \subset U\left(\varphi / A\left[t_{1}, \ldots, t_{n}\right]\right)$. For $n \geq q, n \equiv 1(\bmod q-1)$, we get by (11):

$$
u_{C}\left(t_{1}, \ldots, t_{n} ; 1\right)=e v\left(u_{C}\left(t_{1}, \ldots, t_{n} ; z\right)\right)=\exp _{\varphi}(\mathcal{L}(\varphi / \mathbb{A}))=0 .
$$

Let's set:

$$
\mathbb{B}\left(t_{1}, \ldots, t_{n}\right)=[H(\varphi / \mathbb{A})]_{\mathbb{A}} \in A\left[t_{1}, \ldots, t_{n}\right] .
$$

We recall that if $n \leq 2 q-2$, by [5], Remark 5.3, we have $H\left(\phi / A\left[t_{1}, \ldots, t_{n}\right]\right)=\{0\}$ and therefore $\mathbb{B}\left(t_{1}, \ldots, t_{n}\right)=1$.

3.3.2. The case $n \not \equiv 1(\bmod q-1)$.

In this section, we assume that $n \not \equiv 1(\bmod q-1)$.

Theorem 3.10. $H\left(\varphi / A\left[t_{1}, \ldots, t_{n}\right]\right)$ is a finitely generated and torsion $\mathbb{F}_{q}\left[t_{1}, \ldots, t_{n}\right]$ module. In particular:

$$
\mathbb{B}\left(t_{1}, \ldots, t_{n}\right)=1 .
$$

Proof.

Let $r \in\{2, \ldots, q-1\}$ such that $n \equiv r(\bmod q-1)$. We can assume $n>r$. Set :

$$
N=\left\{x \in \mathbb{T}_{n}\left(K_{\infty}\right), v_{\infty}(x) \geq \frac{n-r}{q-1}\right\} .
$$

Then, since $\frac{n-r}{q-1}>\frac{n-q}{q-1}$, we have :

$$
N=\exp _{\varphi}(N) \subset \exp _{\varphi}\left(\mathbb{T}_{n}\left(K_{\infty}\right)\right)
$$

Observe that:

$$
\mathbb{T}_{n}\left(K_{\infty}\right)=\frac{1}{\theta^{\frac{n-r}{q-1}-1}} A\left[t_{1}, \ldots, t_{n}\right] \oplus N
$$

Set :

$$
E=\frac{\mathbb{T}_{n}\left(K_{\infty}\right)}{A\left[t_{1}, \ldots, t_{n}\right]+N} .
$$

Observe that $E$ is a free and finitely generated $\mathbb{F}_{q}\left[t_{1}, \ldots, t_{n}\right]$-module of rank $\frac{n-r}{q-1}-1$. In order to prove the Theorem, it is enough to prove that there exists $\lambda \in \mathbb{T}_{n}\left(K_{\infty}\right)$, $v_{\infty}(\lambda)=0$, such that if we write in $E$ :

$$
k \in\left\{1, \ldots, \frac{n-r}{q-1}-1\right\}, \exp _{\varphi}\left(\theta^{-k} \lambda\right)=\sum_{j=1}^{\frac{n-r}{q-1}-1} \beta_{j, k}(\lambda) \theta^{-j}, \beta_{j, k}(\lambda) \in \mathbb{F}_{q}\left[t_{1}, \ldots, t_{n}\right],
$$

then $\operatorname{det}\left(\left(\beta_{j, k}(\lambda)\right)_{j, k}\right) \in \mathbb{F}_{q}\left[t_{1}, \ldots, t_{n}\right] \backslash\{0\}$.

Let $\psi: A \rightarrow \mathbb{T}_{n}\left(K_{\infty}\right)\{\tau\}$ be the morphism of $\mathbb{F}_{q}$-algebras given by :

$$
\psi_{\theta}=\left(t_{1}-\theta\right) \cdots\left(t_{r}-\theta\right) \tau+\theta \text {. }
$$

Let $\exp _{\psi}=1+\sum_{j \geq 1} \frac{b_{j}\left(t_{1}\right) \cdots b_{j}\left(t_{r}\right)}{D_{j}} \tau^{j}$. By Lemma 3.7, we have:

$$
\exp _{\psi}(\mathcal{L}(\psi / \mathbb{A}))=1
$$


Set:

$$
\eta=\frac{1}{\omega\left(t_{r+1}\right) \cdots \omega\left(t_{n}\right)} \in \mathbb{T}_{n}\left(K_{\infty}\right)^{\times} .
$$

We refer the reader to Remark 3.3 for the definition of $\omega(t)$. Observe that $v_{\infty}(\eta)=$ $\frac{n-r}{q-1}$. We have :

$$
\forall x \in \mathbb{T}_{n}\left(K_{\infty}\right), \exp _{\phi}(\eta x)=\eta \exp _{\psi}(x)
$$

Thus, for $k \geq 0$ :

$$
\exp _{\phi}\left(\theta^{k} \mathcal{L}(\psi / \mathbb{A}) \eta\right)=\eta \psi_{\theta^{k}}(1) .
$$

We set $\lambda=(-\theta)^{\frac{n-r}{q-1}} \mathcal{L}(\psi / \mathbb{A}) \eta$, note that $\lambda \in \mathbb{T}_{n}\left(K_{\infty}\right)$, and $v_{\infty}(\lambda)=0$. For $k \in$ $\left\{1, \ldots, \frac{n-r}{q-1}-1\right\}$, recall that we write in $E$ :

$$
\exp _{\phi}\left(\theta^{-k} \lambda\right)=\sum_{j=1}^{\frac{n-r}{q-1}-1} \beta_{j, k}(\lambda) \theta^{-j}, \beta_{j, k}(\lambda) \in \mathbb{F}_{q}\left[t_{1}, \ldots, t_{n}\right]
$$

Let $e v_{0}: \mathbb{T}_{n}\left(K_{\infty}\right) \rightarrow K_{\infty}$ be the surjective morphism of $\mathbb{F}_{q}$-algebras given by $e v_{0}(f)=\left.f\right|_{t_{1}=\cdots=t_{n}=0}$. Then $e v_{0}\left((-\theta)^{\frac{n-r}{q-1}} \eta\right)=1$. Let $\varrho: A \rightarrow A\{\tau\}$ be the morphism of $\mathbb{F}_{q}$-algebras given by:

$$
\varrho_{\theta}=\left.\varphi_{\theta}\right|_{t_{1}=\cdots=t_{n}=0}=(-1)^{n} \theta^{n} \tau+\theta .
$$

Then clearly:

We have:

$$
\exp _{\varrho}=\left.\exp _{\varphi}\right|_{t_{1}=\cdots=t_{n}=0}
$$

$$
\left\{x \in K_{\infty}, v_{\infty}(x) \geq \frac{n-r}{q-1}\right\}=e v_{0}(N) \subset \exp _{\varrho}\left(K_{\infty}\right) .
$$

Furthermore $E^{\prime}:=\frac{K_{\infty}}{A \oplus e v_{0}(N)}$ is a finite dimensional $\mathbb{F}_{q}$-vector space of dimension $\frac{n-r}{q-1}-1$. Therefore, for $k \in\left\{1, \ldots, \frac{n-r}{q-1}-1\right\}$, we have in $E^{\prime}$ :

$$
\exp _{\varrho}\left(\theta^{-k}\right)=\sum_{j=1}^{\frac{n-r}{q-1}-1} e v_{0}\left(\beta_{j, k}(\lambda)\right) \theta^{-j}
$$

A direct computation yields for $k \geq 0$ :

$$
e v_{0}\left(\exp _{\varphi}\left(\theta^{k} \mathcal{L}(\psi / \mathbb{A}) \eta\right)\right) \in(-\theta)^{-\frac{n-r}{q-1}} \theta^{k}+(-\theta)^{k+1-\frac{n-r}{q-1}} A
$$

This implies: for $k, j \in\left\{1, \ldots, \frac{n-r}{q-1}-1\right\}, e v_{0}\left(\beta_{k, k}(\lambda)\right) \neq 0$ and $e v_{0}\left(\beta_{j, k}(\lambda)\right)=0$ if $j>k$. Thus $\operatorname{det}\left(\left(e v_{0}\left(\beta_{j, k}(\lambda)\right)_{j, k}\right) \in \mathbb{F}_{q}^{\times}\right.$, and therefore:

$$
\operatorname{det}\left(\left(\beta_{j, k}(\lambda)\right)_{j, k}\right) \in \mathbb{F}_{q}\left[t_{1}, \ldots, t_{n}\right] \backslash\{0\} .
$$

The last assertion of the Theorem is then a consequence of (6)

Let's set:

$$
\mathcal{M}\left(\varphi / A\left[t_{1}, \ldots, t_{n}\right]\right)=\left\{\varphi_{a}\left(u_{C}\left(t_{1}, \ldots, t_{n} ; 1\right)\right), a \in A\left[t_{1}, \ldots, t_{n}\right]\right\},
$$

and its radical $\sqrt{\mathcal{M}\left(\varphi / A\left[t_{1}, \ldots, t_{n}\right]\right)}$ is defined as

$$
\left\{b \in A\left[t_{1}, \ldots, t_{n}\right], \exists a \in A\left[t_{1}, \ldots, t_{n}\right] \backslash\{0\}, \varphi_{a}(b) \in \mathcal{M}\left(\phi / A\left[t_{1}, \ldots, t_{n}\right]\right)\right\} .
$$

Note that, for $n \geq 1, \mathcal{M}\left(\varphi / A\left[t_{1}, \ldots, t_{n}\right]\right)$ is a free $A\left[t_{1}, \ldots, t_{n}\right]$-module of rank one. We will need the following Lemma: 
Lemma 3.11. Let $n \geq 2$ be an integer (we don't assume in this Lemma that $n \not \equiv 1(\bmod q-1))$. Let $u \in A\left[t_{1}, \ldots, t_{n}\right] \backslash\{0\}$ be such that its leading coefficient as a polynomial in $\theta$ is in $\mathbb{F}_{q}^{\times}$. Let $c \in \mathbb{A}$ such that $\varphi_{c}(u) \in A\left[t_{1}, \ldots, t_{n}\right]$, then $c \in A\left[t_{1}, \ldots, t_{n}\right]$.

Proof.

For $k \geq 1$, let's write:

$$
\varphi_{\theta^{k}}=\sum_{j=0}^{k}\left[\theta^{k}, j\right] \tau^{j},\left[\theta^{k}, j\right] \in A\left[t_{1}, \ldots, t_{n}\right] .
$$

Then:

$$
\operatorname{deg}_{\theta}\left[\theta^{k}, j\right]=q^{j}(k-j)+n\left(\frac{q^{j}-1}{q-1}\right),
$$

and the leading coefficient of $\left[\theta^{k}, j\right]$ (viewed as a polynomial in $\theta$ ) lies in $\mathbb{F}_{q}^{\times}$. Observe that, since $n \geq 2$, we have:

$$
j=0, \ldots, k-1, \operatorname{deg}_{\theta}\left[\theta^{k}, k\right]>\operatorname{deg}_{\theta}\left[\theta^{k}, j\right] .
$$

This implies that if $a, b \in A\left[t_{1}, \ldots, t_{n}\right] \backslash\{0\}$, we have:

$$
\operatorname{deg}_{\theta} \varphi_{a}(b)=q^{\operatorname{deg}_{\theta}(a)} \operatorname{deg}_{\theta} b+n\left(\frac{q^{\operatorname{deg}_{\theta} \mathrm{a}}-1}{q-1}\right),
$$

and the leading coefficient of $\varphi_{a}(b)$ viewed as a polynomial in $\theta$ is (up to an element in $\mathbb{F}_{q}^{\times}$) the leading coefficient of $a$ times the leading coefficient of $b$.

Write $c=\delta^{-1} a, \delta \in \mathbb{F}_{q}\left[t_{1}, \ldots, t_{n}\right] \backslash\{0\}$ and $a \in A\left[t_{1}, \ldots, t_{n}\right]$. Write $a=\delta b+d$, where $b, d \in A\left[t_{1}, \ldots, t_{n}\right]$ such that $d=0$ or the leading coefficient of $d$ is not divisible by $\delta$. Then:

$$
\varphi_{d}(u) \in \delta A\left[t_{1}, \ldots, t_{n}\right] .
$$

But, by the above discussion, if $d \neq 0$, the leading coefficient of $\varphi_{d}(u)$ is equal (up to an element in $\mathbb{F}_{q}^{\times}$) to the leading coefficient of $d$. Thus $\delta$ would divide the leading coefficient of $d$ which is a contradiction and therefore $d=0$ and $c \in A\left[t_{1}, \ldots, t_{n}\right]$.

Corollary 3.12. Let $n \geq 1, n \not \equiv 1(\bmod q-1)$. We have:

$$
\sqrt{\mathcal{M}\left(\varphi / A\left[t_{1}, \ldots, t_{n}\right]\right)}=\mathcal{M}\left(\varphi / A\left[t_{1}, \ldots, t_{n}\right]\right) .
$$

Proof. Let $b \in \sqrt{\mathcal{M}\left(\varphi / A\left[t_{1}, \ldots, t_{n}\right]\right)} \backslash\{0\}$, then there exist $a, c \in A\left[t_{1}, \ldots, t_{n}\right] \backslash\{0\}$, such that:

$$
\varphi_{a}(b)=\varphi_{c}\left(u_{C}\left(t_{1}, \ldots, t_{n} ; 1\right)\right) .
$$

By Proposition 3.8, the leading coefficient of $u_{C}\left(t_{1}, \ldots, t_{n} ; 1\right)$ is in $\mathbb{F}_{q}^{\times}$. By (11):

$$
u_{C}\left(t_{1}, \ldots, t_{n} ; 1\right)=\exp _{\varphi}(\mathcal{L}(\varphi / \mathbb{A})) .
$$

Since (recall that $n \not \equiv 1(\bmod q-1)) \varphi_{a}: \mathbb{K}_{\infty} \rightarrow \mathbb{K}_{\infty}$ is an injective map, we get:

$$
b=\exp _{\varphi}\left(\frac{c \mathcal{L}(\varphi / \mathbb{A})}{a}\right) .
$$

But by Theorem 3.10 and (5), we have:

$$
\exp _{\varphi^{-1}}(\mathbb{A})=\mathcal{L}(\varphi / \mathbb{A}) \mathbb{A} .
$$

This implies:

$$
\frac{c \mathcal{L}(\varphi / \mathbb{A})}{a} \in \mathcal{L}(\varphi / \mathbb{A}) \mathbb{A}
$$


Thus $a$ must divide $c$ in $\mathbb{A}$. By Lemma 3.11, we have that $a$ divides $c$ in $A\left[t_{1}, \ldots, t_{n}\right]$ and therefore $b \in \mathcal{M}\left(\varphi / A\left[t_{1}, \ldots, t_{n}\right]\right)$.

Remark 3.13. We briefly treat the case $n=0$. In this case $\varphi=C$ is the Carlitz module and $\mathcal{M}(C / A)=\left\{C_{a}(1), a \in A\right\}$. Observe that:

$$
K_{\infty}=\log _{C}(1) A \oplus M_{\infty},
$$

where $M_{\infty}=\left\{x \in K_{\infty}, v_{\infty}(x) \geq 1\right\}$ and where $\log _{C} \in K\{\{\tau\}\}$ is the Carlitz logarithm (see [13], section 3.4). Therefore:

$$
\exp _{C}\left(K_{\infty}\right)=\mathcal{M}(C / A) \oplus M_{\infty}
$$

This implies:

$$
A \cap \exp _{C}\left(K_{\infty}\right)=\mathcal{M}(C / A)
$$

Thus:

$$
\sqrt{\mathcal{M}(C / A)}=\mathcal{M}(C / A) \text {. }
$$

Furthermore observe that if $q \geq 3, \mathcal{M}(C / A)$ is a free $A$-module of rank one and if $q=2$, we have an isomorphismm of $A$-modules:

$$
\mathcal{M}(C / A) \simeq \frac{A}{(\theta(\theta+1)) A} .
$$

3.3.3. The case $n \equiv 1(\bmod q-1)$.

In this section, we assume that $n \geq q$ and $n \equiv 1(\bmod q-1)$. Recall that, by Lemma 3.9, we have :

$$
u_{C}\left(t_{1}, \ldots, t_{n} ; 1\right)=0 \text {. }
$$

Therefore, for $n \geq q$, we set:

$$
u_{C}^{(1)}\left(t_{1}, \ldots, t_{n} ; z\right)=\frac{d}{d z} u_{C}\left(t_{1}, \ldots, t_{n} ; z\right) \in A\left[t_{1}, \ldots, t_{n}, z\right] .
$$

Observe that, if we set $N=\left\{x \in \mathbb{T}_{n}\left(K_{\infty}\right), v_{\infty}(x)>\frac{n-q}{q-1}\right\}$, then:

$$
\mathbb{T}_{n}\left(K_{\infty}\right)=\frac{\tilde{\pi}}{\omega\left(t_{1}\right) \cdots \omega\left(t_{n}\right)} A\left[t_{1}, \ldots, t_{n}\right] \oplus N .
$$

Furthermore, by (12), we get:

$$
N=\exp _{\varphi}(N)=\exp _{\varphi}\left(\mathbb{T}_{n}\left(K_{\infty}\right)\right)
$$

\section{Proposition 3.14.}

We set $E=\frac{\mathbb{T}_{n}\left(K_{\infty}\right)}{N}$ viewed as an $A\left[t_{1}, \ldots, t_{n}\right]$-module via $\varphi$. Then $\exp _{\varphi}^{(1)}$ induces an injective morphism of $A\left[t_{1}, \ldots, t_{n}\right]$-modules :

$$
\exp _{\varphi}^{(1)}: E \hookrightarrow E
$$

Proof. Recall that by (12), we have:

$$
U\left(\varphi / A\left[t_{1}, \ldots, t_{n}\right]\right)=\frac{\widetilde{\pi}}{\omega\left(t_{1}\right) \cdots \omega\left(t_{n}\right)} A\left[t_{1}, \ldots, t_{n}\right] .
$$

By (11), we have:

$$
\exp _{\varphi}^{(1)}(\mathcal{L}(\varphi / \mathbb{A})) \equiv u_{C}^{(1)}\left(t_{1}, \ldots, t_{n} ; 1\right) \quad(\bmod N)
$$

Furthermore, for $x \in U\left(\varphi / A\left[t_{1}, \ldots, t_{n}\right]\right)$, using the fact that $\exp _{\widetilde{\varphi}} \theta=\widetilde{\varphi}_{\theta} \exp _{\widetilde{\varphi}}$ and that $\exp _{\varphi}(x)=0$, we have:

$$
\exp _{\varphi}^{(1)}(\theta x) \equiv \varphi_{\theta}\left(\exp _{\varphi}^{(1)}(x)\right) \quad(\bmod N)
$$


Also observe that:

$$
\exp _{\varphi}^{(1)}(N) \subset N
$$

Let $x \in \mathbb{T}_{n}\left(K_{\infty}\right)$ such that there exists $a \in A\left[t_{1}, \ldots, t_{n}\right] \backslash\{0\}, a$ monic as a polynomial in $\theta$, with $\varphi_{a}(x)=0$ in $E$. Then, by [5], proof of Corollary 6.5, we have:

$$
x \in \exp _{\varphi}\left(T_{n}\left(K_{\infty}\right)\right)=N .
$$

By Proposition 3.8 we have that $u_{C}^{(1)}\left(t_{1}, \ldots, t_{n} ; 1\right) \in A\left[t_{1}, \ldots, t_{n}\right] \backslash\{0\}$, and since $\varphi_{\mathbb{B}\left(t_{1}, \ldots, t_{n}\right)}\left(\exp _{\varphi}^{(1)}\left(U\left(\varphi / A\left[t_{1}, \ldots, t_{n}\right]\right)\right) \equiv u_{C}^{(1)}\left(t_{1}, \ldots, t_{n} ; 1\right) A\left[t_{1}, \ldots, t_{n}\right]\right) \quad(\bmod N)$, and $A\left[t_{1}, \ldots, t_{n}\right] \cap N=\{0\}$, we deduce the assertion of the Proposition.

Lemma 3.15. We have:

$$
\exp _{\varphi}^{(1)}\left(\frac{\tilde{\pi}}{\omega\left(t_{1}\right) \cdots \omega\left(t_{n}\right)}\right) \equiv \frac{(-1)^{\frac{n-q}{q-1}}}{\theta^{\frac{n-q}{q-1}}}(\bmod N)
$$

Proof. We first notice that :

$$
v_{\infty}\left(\frac{\tilde{\pi}}{\omega\left(t_{1}\right) \cdots \omega\left(t_{n}\right)}-\frac{(-1)^{\frac{n-q}{q-1}}}{\theta^{\frac{n-q}{q-1}}}\right)>\frac{n-q}{q-1} .
$$

For $i \geq 2$, we also have that:

$$
v_{\infty}\left(\frac{b_{i}\left(t_{1}\right) \cdots b_{i}\left(t_{n}\right) \tau^{i}\left(\frac{\widetilde{\pi}}{\omega\left(t_{1}\right) \cdots \omega\left(t_{n}\right)}\right)}{D_{i}}\right)>\frac{n-q}{q-1} .
$$

Furthermore, observe that:

$$
\tau\left(\frac{\tilde{\pi}}{\omega\left(t_{1}\right) \ldots \omega\left(t_{n}\right)}\right)=\frac{\tilde{\pi}^{q}}{\left(t_{1}-\theta\right) \cdots\left(t_{n}-\theta\right) \omega\left(t_{1}\right) \cdots \omega\left(t_{n}\right)} .
$$

Finally, observe that :

$$
v_{\infty}\left(\frac{\widetilde{\pi}^{q}}{\left(\theta^{q}-\theta\right) \omega\left(t_{1}\right) \cdots \omega\left(t_{n}\right)}-\frac{(-1)^{\frac{n-q}{q-1}}}{\theta^{\frac{n-q}{q-1}}}\right)>\frac{n-q}{q-1} .
$$

Lemma 3.16. Let $a \in A\left[t_{1}, \ldots, t_{n}\right] \backslash\{0\}$, a monic as a polynomial in $\theta$. If

$$
\varphi_{a}\left(\frac{1}{\theta^{\frac{n-q}{q-1}}}\right) \in A\left[t_{1}, \ldots, t_{n}\right]+\exp _{\varphi}\left(\mathbb{T}_{n}\left(K_{\infty}\right)\right),
$$

then $\operatorname{deg}_{\theta}(a) \geq \frac{n-q}{q-1}$.

Proof. Write $a=\theta^{r}+\sum_{i=0}^{r-1} a_{i} \theta^{i}, a_{i} \in \mathbb{F}_{q}\left[t_{1}, \ldots, t_{n}\right]$. Set $b=\left.a\right|_{t_{j}=0, j=1, \cdots n}$. Let $\varrho: A \rightarrow A\{\tau\}$ be the Drinfeld $A$-module of rank one given by $\varrho_{\theta}=(-1)^{n} \theta^{n} \tau+\theta$. We have:

We easily deduce that:

$$
\operatorname{Ker}\left(\exp _{\varrho}: K_{\infty} \rightarrow K_{\infty}\right)=\frac{\widetilde{\pi}}{\exp _{C}\left(\frac{\tilde{\pi}}{\theta}\right)^{n}} A
$$

$$
\exp _{\varrho}\left(K_{\infty}\right)=\left\{x \in K_{\infty}, v_{\infty}(x)>\frac{n-q}{q-1}\right\} .
$$

Since $\varphi_{a}\left(\frac{1}{\theta^{\frac{n-q}{q-1}}}\right) \in A\left[t_{1}, \ldots, t_{n}\right]+\exp _{\varphi}\left(\mathbb{T}_{n}\left(K_{\infty}\right)\right)$, we have :

$$
\varrho_{b}\left(\frac{1}{\theta^{\frac{n-q}{q-1}}}\right) \in A+\exp _{\varrho}\left(K_{\infty}\right) .
$$


We observe that, for $\frac{n-q}{q-1} \geq \ell \geq 1$, we have :

$$
\varrho_{\theta}\left(\frac{1}{\theta^{\ell}}\right) \equiv \frac{1}{\theta^{\ell-1}} \quad\left(\bmod \frac{1}{\theta^{\ell-2}} A\right) .
$$

Thus, for $0 \leq i \leq \frac{n-q}{q-1}-1$, we get:

$$
\varrho_{\theta^{i}}\left(\frac{1}{\theta^{(n-q) /(q-1)}}\right) \equiv \frac{1}{\theta^{(n-q) /(q-1)-i}} \quad\left(\bmod \frac{1}{\theta^{(n-q) /(q-1)-i-1}} A\right) .
$$

This implies that $r \geq \frac{n-q}{q-1}$.

Theorem 3.17. The module $H^{(1)}\left(\varphi / A\left[t_{1}, \ldots, t_{n}\right]\right)$ is the sub- $A\left[t_{1}, \ldots, t_{n}\right]$-module of $H\left(\varphi / A\left[t_{1}, \ldots, t_{n}\right]\right)$ generated by the image of $\frac{1}{\theta^{(n-q) /(q-1)}}$ in $H\left(\varphi / A\left[t_{1}, \ldots, t_{n}\right]\right)$. We have an isomorphism of $A\left[t_{1}, \ldots, t_{n}\right]$-modules:

$$
H^{(1)}\left(\varphi / A\left[t_{1}, \ldots, t_{n}\right]\right) \simeq \frac{A\left[t_{1}, \ldots, t_{n}\right]}{\mathbb{B}\left(t_{1}, \ldots, t_{n}\right) A\left[t_{1}, \ldots, t_{n}\right]} .
$$

Furthermore, $\frac{H\left(\varphi / A\left[t_{1}, \ldots, t_{n}\right]\right)}{H^{(1)}\left(\varphi / A\left[t_{1}, \ldots, t_{n}\right]\right)}$ is a finitely generated and torsion $\mathbb{F}_{q}\left[t_{1}, \ldots, t_{n}\right]$ module.

Proof. Set:

$$
V=\left\{x \in U\left(\varphi / A\left[t_{1}, \ldots, t_{n}\right]\right), \exp _{\varphi}^{(1)}(x) \in A\left[t_{1}, \ldots, t_{n}\right]+N\right\} .
$$

We have:

$$
U_{S t}\left(\varphi / A\left[t_{1}, \ldots, t_{n}\right]\right)=\mathcal{L}(\varphi / \mathbb{A}) A\left[t_{1}, \ldots, t_{n}\right] \subset V .
$$

Observe that the $k$-vector space $W$ generated by $V$ is a free $\mathbb{A}$-module of rank one and, by (5), that $G:=\left[\frac{U(\varphi / \mathbb{A})}{W}\right]_{\mathbb{A}}$ divides $\mathbb{B}\left(t_{1}, \ldots, t_{n}\right)$ in $\mathbb{A}$. Thus $G \in A\left[t_{1}, \ldots, t_{n}\right]$. This implies:

$$
V=G U\left(\varphi / A\left[t_{1}, \ldots, t_{n}\right]\right) .
$$

We have :

$$
\exp _{\varphi}^{(1)}\left(\frac{G \widetilde{\pi}}{\omega\left(t_{1}\right) \cdots \omega\left(t_{n}\right)}\right) \in A\left[t_{1}, \ldots, t_{n}\right]+N .
$$

Therefore, by Lemma 3.15

$$
\varphi_{G}\left(\frac{1}{\theta^{\frac{n-q}{q-1}}}\right) \in A\left[t_{1}, \ldots, t_{n}\right]+N
$$

Thus by Lemma 3.16. we get that $\operatorname{deg}_{\theta} G \geq \frac{n-q}{q-1}$, and since $G$ divides $\mathbb{B}\left(t_{1}, \ldots, t_{n}\right)$, we get $G=\mathbb{B}\left(t_{1}, \ldots, t_{n}\right)$, i.e.:

$$
V=U_{S t}\left(\varphi / A\left[t_{1}, \ldots, t_{n}\right]\right) .
$$

By Proposition 3.14 and the above equality, the $\operatorname{map} \exp _{\varphi}^{(1)}$ induces an injective morphism of $A\left[t_{1}, \ldots, t_{n}\right]$-modules:

$$
\frac{U\left(\varphi / A\left[t_{1}, \ldots, t_{n}\right]\right)}{U_{S t}\left(\varphi / A\left[t_{1}, \ldots, t_{n}\right]\right)} \hookrightarrow H\left(\varphi / A\left[t_{1}, \ldots, t_{n}\right]\right) .
$$


Let's set (recall that $n \geq q)$ :

$$
\mathcal{M}\left(\varphi / A\left[t_{1}, \ldots, t_{n}\right]\right)=\left\{\varphi_{a}\left(u_{C}^{(1)}\left(t_{1}, \ldots, t_{n} ; 1\right)\right), a \in A\left[t_{1}, \ldots, t_{n}\right]\right\},
$$

and define its radical $\sqrt{\mathcal{M}\left(\varphi / A\left[t_{1}, \ldots, t_{n}\right]\right)}$ by

$$
\left\{x \in A\left[t_{1}, \ldots, t_{n}\right], \exists a \in A\left[t_{1}, \ldots, t_{n}\right] \backslash\{0\}, \varphi_{a}(x) \in \mathcal{M}\left(\varphi / A\left[t_{1}, \ldots, t_{n}\right]\right)\right\} .
$$

By the proof of Proposition [3.14, $\mathcal{M}\left(\varphi / A\left[t_{1}, \ldots, t_{n}\right]\right)$ is a free $A\left[t_{1}, \ldots, t_{n}\right]$-module of rank one.

Corollary 3.18. We have:

$$
\sqrt{\mathcal{M}\left(\varphi / A\left[t_{1}, \ldots, t_{n}\right]\right)}=\mathcal{M}\left(\varphi / A\left[t_{1}, \ldots, t_{n}\right]\right) .
$$

Proof. Let $b \in \sqrt{\mathcal{M}\left(\varphi / A\left[t_{1}, \ldots, t_{n}\right]\right)} \backslash\{0\}$, then there exist $a, c \in A\left[t_{1}, \ldots, t_{n}\right] \backslash\{0\}$, such that:

$$
\varphi_{a}(b)=\varphi_{c}\left(u_{C}^{(1)}\left(t_{1}, \ldots, t_{n} ; 1\right)\right) \text {. }
$$

Let $E^{\prime}=\frac{\mathbb{K}_{\infty}}{N^{\prime}}$ where $N^{\prime}=\left\{x \in \mathbb{K}_{\infty}, v_{\infty}(x)>\frac{n-q}{q-1}\right\}$. By an adaptation of the proofs of Proposition 3.14 and Theorem 3.17. we have:

$$
\mathcal{L}(\varphi / \mathbb{A}) \mathbb{A}+N^{\prime}=\left\{x \in \mathbb{K}_{\infty}, \exp _{\varphi}^{(1)}(x) \in \mathbb{A}+N^{\prime}\right\} .
$$

This implies that $\exp _{\varphi}^{(1)}$ induces an isomorphism of $k$-vector spaces:

$$
\frac{\mathbb{K}_{\infty}}{\mathcal{L}(\varphi / \mathbb{A}) \mathbb{A}+N^{\prime}} \simeq \frac{\mathbb{K}_{\infty}}{\mathbb{A}+N^{\prime}}
$$

Therefore, there exists $d \in \mathbb{A}$ such that we have the following equality in $E^{\prime}$ :

$$
b=\varphi_{d}\left(u_{C}^{(1)}\left(t_{1}, \ldots, t_{n} ; 1\right)\right) .
$$

Since $b, u_{C}^{(1)}\left(t_{1}, \ldots, t_{n} ; 1\right) \in \mathbb{A}$, this implies the equality in $\mathbb{K}_{\infty}$ :

$$
b=\varphi_{d}\left(u_{C}^{(1)}\left(t_{1}, \ldots, t_{n} ; 1\right)\right) .
$$

By an adaptation of the proof of Proposition 3.14, for $x \in \mathbb{A} \backslash\{0\}, \phi_{x}: E^{\prime} \rightarrow E^{\prime}$ is an injective morphism of $\mathbb{A}$-modules. In particular, we get:

$$
a d=c .
$$

Again, since $E^{\prime}$ has no torsion point for $\varphi$, we get in $E^{\prime}$ :

$$
b=\varphi_{c / a}\left(u_{C}^{(1)}\left(t_{1}, \ldots, t_{n} ; 1\right)\right) .
$$

Since $\mathbb{A} \cap N^{\prime}=\{0\}$, this equality is also true in $\mathbb{K}_{\infty}$. By Proposition 3.8, the leading coefficient of $u_{C}^{(1)}\left(t_{1}, \ldots, t_{n} ; 1\right)$ is in $\mathbb{F}_{q}^{\times}$. Now apply Lemma 3.11, we get $\frac{c}{a} \in A\left[t_{1}, \ldots, t_{n}\right]$ and therefore $b \in \mathcal{M}\left(\varphi / A\left[t_{1}, \ldots, t_{n}\right]\right)$.

Remark 3.19. When $n=1$, then we already mentioned that $H\left(\phi / A\left[t_{1}\right]\right)=\{0\}$. Furthermore, by Lemma 3.7, we have:

$$
u_{C}\left(t_{1} ; 1\right)=1 .
$$

So we set, in this case:

$$
\begin{gathered}
\mathcal{M}\left(\varphi / A\left[t_{1}\right]\right)=\left\{\varphi_{a}(1), a \in A\left[t_{1}\right]\right\} \\
\sqrt{\mathcal{M}\left(\varphi / A\left[t_{1}\right]\right)}=\left\{x \in A\left[t_{1}\right], \exists b \in A\left[t_{1}\right] \backslash\{0\}, \varphi_{b}(x) \in \mathcal{M}\left(\varphi / A\left[t_{1}\right]\right)\right\} .
\end{gathered}
$$

We have:

$$
\varphi_{\theta-t_{1}}(1)=0 .
$$




\section{Furthermore:}

$$
U(\varphi / \mathbb{A})=\mathcal{L}(\varphi / \mathbb{A}) \mathbb{A}
$$

and:

$$
\operatorname{Ker}\left(\exp _{\phi}: \mathbb{T}_{1}\left(K_{\infty}\right) \rightarrow \mathbb{T}_{1}\left(K_{\infty}\right)\right)=\frac{\tilde{\pi}}{\omega\left(t_{1}\right)} A\left[t_{1}\right]
$$

By [5, Lemma \%.1, we have an isomorphism of $A\left[t_{1}\right]$-modules:

$$
\mathcal{M}\left(\varphi / A\left[t_{1}\right]\right) \simeq \frac{A\left[t_{1}\right]}{\left(\theta-t_{1}\right) A\left[t_{1}\right]} .
$$

In particular $\mathcal{M}\left(\varphi / A\left[t_{1}\right]\right)=\mathbb{F}_{q}\left[t_{1}\right]$. But, since $\mathcal{M}\left(\varphi / A\left[t_{1}\right]\right) \subset \exp _{\varphi}\left(\mathbb{T}_{1}\left(K_{\infty}\right)\right)$, we get: $\sqrt{\mathcal{M}\left(\varphi / A\left[t_{1}\right]\right)} \subset \exp _{\varphi}\left(\mathbb{K}_{\infty}\right) \cap \mathbb{A}=\exp _{\varphi}(U(\varphi / \mathbb{A}))$. Thus: $\sqrt{\mathcal{M}\left(\varphi / A\left[t_{1}\right]\right)} \subset$ $\exp _{\varphi}(U(\varphi / \mathbb{A}))$. Note that we have an isomorphism of $\mathbb{A}$-modules:

$$
\exp _{\varphi}(U(\varphi / \mathbb{A})) \simeq \frac{\mathbb{A}}{\left(\theta-t_{1}\right) \mathbb{A}}
$$

and $\exp _{\varphi}(U(\varphi / \mathbb{A}))$ is the $\mathbb{A}$-module (via $\varphi$ ) generated by 1 . In particular, we have $\exp _{\varphi}(U(\varphi / \mathbb{A}))=\mathbb{F}_{q}\left(t_{1}\right)$. Thus:

$$
\exp _{\varphi}(U(\varphi / \mathbb{A})) \cap \mathbb{T}_{1}\left(K_{\infty}\right)=\mathcal{M}\left(\varphi / A\left[t_{1}\right]\right) .
$$

Therefore, we also have in this case:

$$
\sqrt{\mathcal{M}\left(\varphi / A\left[t_{1}\right]\right)}=\mathcal{M}\left(\varphi / A\left[t_{1}\right]\right)
$$

\section{4. $P$-adic $L$-series.}

Let $n \geq 0$ be an integer. Let $\phi: A \rightarrow A\{\tau\}$ be a Drinfeld $A$-module defined over $A$, and let $\varphi$ be its canonical deformation over $\mathbb{T}_{n}\left(K_{\infty}\right), \widetilde{\varphi}$ be the canonical $z$-deformation of $\varphi$ (see section 3.1).

Let $\log _{\phi} \in K\{\{\tau\}\}$ (respectively $\log _{\varphi} \in \mathbb{T}_{n}\left(K_{\infty}\right)\{\{\tau\}\}, \log _{\widetilde{\varphi}} \in \mathbb{T}_{n, z}\left(K_{\infty}\right)\{\{\tau\}\}$ ) be the unique element such that $\log _{\phi} \equiv 1(\bmod \tau)$ and :

$$
\log _{\phi} \phi_{\theta}=\theta \log _{\phi}\left(\text { respectively } \log _{\varphi} \varphi_{\theta}=\theta \log _{\varphi}, \log _{\widetilde{\varphi}} \widetilde{\varphi}_{\theta}=\theta \log _{\widetilde{\varphi}}\right)
$$

Write:

$$
\log _{\phi}=\sum_{j \geq 0} l_{j} \tau^{j}, l_{j} \in K
$$

then:

$$
\log _{\varphi}=\sum_{j \geq 0} b_{j}\left(t_{1}\right) \ldots b_{j}\left(t_{n}\right) l_{j} \tau^{j} \text { and } \log _{\widetilde{\varphi}}=\sum_{j \geq 0} b_{j}\left(t_{1}\right) \ldots b_{j}\left(t_{n}\right) l_{j} z^{j} \tau^{j} .
$$

Now, we fix $P$ a monic irreducible element of $A$ of degree $d \geq 1$. Let $v_{P}: K \rightarrow$ $\mathbb{Z} \cup\{+\infty\}$ be the $P$-adic valuation on $K$.

Lemma 3.20. For $n \geq 0$, we have:

$$
v_{P}\left(l_{n}\right) \geq-\left[\frac{n}{d}\right]
$$

where for $x \in \mathbb{R},[x]$ denotes the integer part of $x$.

Proof. We have for $n \geq 1$ :

$$
\left(\theta-\theta^{q^{n}}\right) l_{n}=\sum_{j=0}^{n-1} l_{j} \alpha_{n-j}^{q^{j}},
$$


where $\phi_{\theta}=\sum_{j \geq 0} \alpha_{j} \tau^{j}$, and $\alpha_{j} \in A, \alpha_{j}=0$ for $j \geq r+1$ where $r$ is the rank of $\phi$. Set $\ell_{0}=1$ and for $j \geq 1, \ell_{j}=\prod_{k=1}^{j-1}\left(\theta-\theta^{q^{k}}\right)$. Then, we deduce that:

$$
\forall n \geq 0, l_{n}=\frac{a_{n}}{\ell_{n}}, a_{n} \in A .
$$

Let $K_{P}^{a c}$ be an algebraic closure of $K_{P}$, where $K_{P}$ is the $P$-adic completion of $K$. Let $\mathbb{C}_{P}$ be the completion of $K_{P}^{a c}$ equipped with the $P$-adic valuation $v_{P}$ : $\mathbb{C}_{P} \rightarrow \mathbb{Q} \cup\{+\infty\}\left(v_{P}(P)=1\right)$. We denote by $v_{P}$ the $P$-adic Gauss valuation on $\mathbb{C}_{P}\left[t_{1}, \ldots, t_{n}, z\right]$, i.e. if $f=\sum_{i_{1}, \ldots, i_{n}, j \in \mathbb{N}} x_{i_{1}, \ldots, i_{n}, j} t_{1}^{i_{1}} \cdots t_{n}^{i_{n}} z^{j}, x_{i_{1}, \ldots, i_{n}, j} \in \mathbb{C}_{P}$, then

$$
v_{P}(f)=\operatorname{Inf}\left\{v_{P}\left(x_{i_{1}, \ldots, i_{n}, j}\right), i_{1}, \ldots, i_{n}, j \in \mathbb{N}\right\} .
$$

Let $\mathbb{T}_{n, z}\left(\mathbb{C}_{P}\right)$ be the completion of $\mathbb{C}_{P}\left[t_{1}, \ldots, t_{n}, z\right]$ for $v_{P}$, and let $\mathbb{T}_{n}\left(\mathbb{C}_{P}\right)$ be the closure of $\mathbb{C}_{P}\left[t_{1}, \ldots, t_{n}\right]$ in $\mathbb{T}_{n, z}\left(\mathbb{C}_{P}\right)$.

We denote by $\mathbb{T}_{n, z}\left(K_{P}\right)$ the closure of $K\left[t_{1}, \ldots, t_{n}, z\right]$ in $\mathbb{T}_{n, z}\left(\mathbb{C}_{P}\right)$, and $\mathbb{T}_{n}\left(K_{P}\right)$ the closure of $K\left[t_{1}, \ldots, t_{n}\right]$ in $\mathbb{T}_{n, z}\left(\mathbb{C}_{P}\right)$. We also denote by $\mathbb{A}_{P}$ the closure of $A\left[t_{1}, \ldots, t_{n}\right]$ in $\mathbb{T}_{n}\left(K_{P}\right)$, and $\widetilde{\mathbb{A}_{P}}$ the closure of $A\left[t_{1}, \ldots, t_{n}, z\right]$ in $\mathbb{T}_{n, z}\left(K_{P}\right)$. Finally let $O=\left\{x \in \mathbb{C}_{P}, v_{P}(x) \geq 0\right\}$, and let $\widetilde{\mathbb{O}}$ be the closure of $O\left[t_{1}, \ldots, t_{n}, z\right]$ in $\mathbb{T}_{n, z}\left(\mathbb{C}_{P}\right)$, and $\mathbb{O}$ be the closure of $O\left[t_{1}, \ldots, t_{n}\right]$ in $\mathbb{T}_{n, z}\left(\mathbb{C}_{P}\right)$.

We still denote by $\tau$ the continuous morphism of $\mathbb{F}_{q}\left(t_{1}, \ldots, t_{n}, z\right)$-algebras $\tau$ : $\mathbb{T}_{n, z}\left(\mathbb{C}_{P}\right) \rightarrow \mathbb{T}_{n, z}\left(\mathbb{C}_{P}\right), \forall x \in \mathbb{C}_{P}, \tau(x)=x^{q}$.

Lemma 3.21. $\widetilde{\varphi}$ extends by continuity to a morphism of $\mathbb{F}_{q}\left[t_{1}, \ldots, t_{n}, z\right]$-algebras :

$$
\widetilde{\varphi}: \widetilde{\mathbb{A}_{P}} \rightarrow \widetilde{\mathbb{A}_{P}}\{\{\tau\}\} \text {. }
$$

Proof. Observe that:

$$
\widetilde{\varphi}_{P}=P+f \tau, f \in A\left[t_{1}, \ldots, t_{n}, z\right]\{\tau\} .
$$

This implies that, for $m \geq 1$, we have:

$$
\widetilde{\varphi}_{P^{m}}=\sum_{k=0}^{m-1} a_{k} \tau^{k}+f_{m} \tau^{m}, a_{0}, \ldots, a_{m-1} \in A\left[t_{1}, \ldots, t_{n}, z\right], f_{m} \in A\left[t_{1}, \ldots, t_{n}, z\right]\{\tau\},
$$

with the property:

$$
k \in\{0, \ldots, m-1\}, v_{P}\left(a_{k}\right) \geq m-k .
$$

This implies that if $x \in P^{m} A\left[t_{1}, \ldots, t_{n}, z\right]$ for some $m \geq 1$, we get:

$$
\widetilde{\varphi}_{x}=\sum_{k=0}^{m-1} b_{k} \tau^{k}+g_{m} \tau^{m}, b_{0}, \ldots, b_{m-1} \in A\left[t_{1}, \ldots, t_{n}, z\right], g_{m} \in A\left[t_{1}, \ldots, t_{n}, z\right]\{\tau\},
$$

with the property:

$$
k \in\{0, \ldots, m-1\}, v_{P}\left(b_{k}\right) \geq m-k .
$$

Thus, if $\left(a_{n}\right)_{n \geq 0}$ is a Cauchy sequence for $v_{P}$ of elements in $A\left[t_{1}, \ldots, t_{n}, z\right]$, then the sequence $\left(\widetilde{\varphi}_{a_{n}}\right)_{n \geq 0}$ converges in $\widetilde{\mathbb{A}_{P}}\{\{\tau\}\}$.

Observe that $\widetilde{\mathbb{O}}$ is an $A\left[t_{1}, \ldots, t_{n}, z\right]$-module via $\widetilde{\varphi}$, and by the above Lemma $\left\{x \in \widetilde{\mathbb{O}}, v_{P}(x)>0\right\}$ is an $\widetilde{\mathbb{A}_{P}}$-module via $\widetilde{\varphi}$. 
Lemma 3.22. $\log _{\widetilde{\varphi}}$ induces an injective morphism of $\mathbb{F}_{q}\left[t_{1}, \ldots, t_{n}, z\right]$-modules:

$$
\log _{\widetilde{\varphi}, P}: \widetilde{\mathbb{O}} \rightarrow \mathbb{T}_{n, z}\left(\mathbb{C}_{P}\right),
$$

such that:

$$
\forall a \in A\left[t_{1}, \ldots, t_{n}, z\right], \forall x \in \widetilde{\mathbb{O}}, \log _{\widetilde{\varphi}, P}\left(\widetilde{\varphi}_{a}(x)\right)=a \log _{\widetilde{\varphi}, P}(x) .
$$

Furthermore, if $x \in \widetilde{\mathbb{O}}, v_{P}(x)>0$, we have:

$$
\log _{\widetilde{\varphi}, P}(x)=\sum_{j \geq 0} b_{j}\left(t_{1}\right) \cdots b_{j}\left(t_{n}\right) z^{j} l_{j} \tau^{j}(x)
$$

and:

$$
\forall a \in \widetilde{\mathbb{A}_{P}}, \log _{\widetilde{\varphi}, P}\left(\widetilde{\varphi}_{a}(x)\right)=a \log _{\widetilde{\varphi}, P}(x)
$$

Proof. Let's set:

$$
\mathcal{M}=\left\{x \in \widetilde{\mathbb{O}}, v_{P}(x)>0\right\} .
$$

Observe that we have a direct sum of $\mathbb{F}_{q}\left[t_{1}, \ldots, t_{n}, z\right]$-modules:

$$
\widetilde{\mathbb{O}}=\overline{\mathbb{F}_{q}}\left[t_{1}, \ldots, t_{n}, z\right] \oplus \mathcal{M},
$$

where $\overline{\mathbb{F}_{q}}$ is the algebraic closure of $\mathbb{F}_{q}$ in $\mathbb{C}_{P}$. By Lemma 3.20, $\log _{\widetilde{\varphi}}$ converges on $\mathcal{M}$ and we have the last assertion of the Lemma by Lemma 3.21. Furthermore, for $x \in \mathcal{M}$, we have:

$$
v_{P}\left(\widetilde{\varphi}_{P}(x)\right) \geq \operatorname{Inf}\left\{q v_{P}(x), v_{P}(x)+1\right\} .
$$

This implies that there exists an integer $m$ depending on $x$ such that:

$$
\log _{\widetilde{\varphi}}(x)=\frac{1}{P^{m}} \log _{\widetilde{\varphi}}\left(\widetilde{\varphi}_{P^{m}}(x)\right) \in \frac{1}{P^{m}} \mathcal{M},
$$

and again by Lemma 3.20

$$
v_{P}\left(\log _{\widetilde{\varphi}}\left(\widetilde{\varphi}_{P^{m}}(x)\right)=v_{P}\left(\widetilde{\varphi}_{P^{m}}(x)\right) .\right.
$$

Now, we observe that $\widetilde{\mathbb{O}}$ is an $A\left[t_{1}, \ldots, t_{n}, z\right]$-module via $\widetilde{\varphi}$ without torsion. This implies that $\log _{\tilde{\varphi}}: \mathcal{M} \rightarrow \mathbb{T}_{n, z}\left(\mathbb{C}_{P}\right)$ is injective.

Now, let $m \geq 1, m \equiv 0(\bmod d)$. Let:

$$
\bar{M}=\frac{\mathbb{F}_{q^{m}}\left[t_{1}, \ldots, t_{n}, z\right] \oplus \mathcal{M}}{\mathcal{M}} .
$$

Then $\bar{M}$ is a finitely generated and a free $\mathbb{F}_{q}\left[t_{1}, \ldots, t_{n}, z\right]$-module and also an $A\left[t_{1}, \ldots, t_{n}, z\right]$-module via $\widetilde{\varphi}$. This implies that $\bar{M}$ is a torsion $A\left[t_{1}, \ldots, t_{n}, z\right]$ module. Thus there exists $a \in A\left[t_{1}, \ldots, t_{n}, z\right] \cap \mathbb{T}_{n, z}\left(K_{P}\right)^{\times}$such that:

$$
\widetilde{\varphi}_{a}\left(\mathbb{F}_{q^{m}}\right) \subset \mathcal{M} .
$$

Now, let $x \in \widetilde{\mathbb{O}}$. By the above discussion, there exists $b \in A\left[t_{1}, \ldots, t_{n}, z\right] \cap \mathbb{T}_{n, z}\left(K_{P}\right)^{\times}$ such that:

$$
\widetilde{\varphi}_{b}(x) \in \mathcal{M} .
$$

We set:

$$
\log _{\widetilde{\varphi}, P}(x)=\frac{1}{b} \log _{\widetilde{\varphi}}\left(\widetilde{\varphi}_{b}(x)\right) .
$$

This does not depend on the choice of $b$. 
Recall that:

$$
\mathcal{L}(\widetilde{\varphi} / \widetilde{\mathbb{A}})=\prod_{Q} \frac{\left[\frac{\widetilde{\mathbb{A}}}{Q \widetilde{\mathbb{A}}}\right]_{\widetilde{\mathbb{A}}}}{\left[\widetilde{\varphi}\left(\frac{\widetilde{\mathbb{A}}}{Q \widetilde{\mathbb{A}}}\right]_{\widetilde{\mathbb{A}}}\right.} \in \mathbb{T}_{n, z}\left(K_{\infty}\right)^{\times}
$$

where $Q$ runs through the monic irreducible elements in $A$. Thus:

$$
\left(\frac{\left[\frac{\widetilde{\mathbb{A}}}{P \widetilde{\mathbb{A}}}\right]_{\widetilde{\mathbb{A}}}}{\left[\widetilde{\varphi}\left(\frac{\widetilde{\mathbb{A}}}{P \widetilde{\mathbb{A}}}\right]_{\widetilde{\mathbb{A}}}\right.}\right)^{-1} \mathcal{L}(\widetilde{\varphi} / \widetilde{\mathbb{A}})=\prod_{Q \neq P} \frac{\left[\frac{\widetilde{\mathbb{A}}}{Q \mathbb{A}}\right]_{\widetilde{\mathbb{A}}}}{\left[\widetilde{\varphi}\left(\frac{\widetilde{\mathbb{A}}}{Q \widetilde{\mathbb{A}}}\right]_{\widetilde{\mathbb{A}}}\right.} \in \mathbb{T}_{n, z}\left(K_{\infty}\right)^{\times} .
$$

Now, by (8), we have the formal equality in $K\left(t_{1}, \ldots, t_{n}\right)[[z]]$ :

$$
\mathcal{L}(\widetilde{\varphi} / \widetilde{\mathbb{A}})=\log _{\widetilde{\varphi}}\left(u_{\phi}\left(t_{1}, \ldots, t_{n} ; z\right)\right) .
$$

Therefore, we define $\mathcal{L}_{P}(\widetilde{\varphi} / \widetilde{\mathbb{A}})$ by the formal equality in $K\left(t_{1}, \ldots, t_{n}\right)[[z]]$ :

$$
\mathcal{L}_{P}(\widetilde{\varphi} / \widetilde{\mathbb{A}})=\left(\frac{\left[\frac{\widetilde{\mathbb{A}}}{P \widetilde{\mathbb{A}}}\right]_{\widetilde{\mathbb{A}}}}{\left[\widetilde{\varphi}\left(\frac{\widetilde{\mathbb{A}}}{P \widetilde{\mathbb{A}}}\right)\right]_{\widetilde{\mathbb{A}}}}\right)^{-1} \mathcal{L}(\widetilde{\varphi} / \widetilde{\mathbb{A}}) .
$$

Theorem 3.23. $\mathcal{L}_{P}(\widetilde{\varphi} / \widetilde{\mathbb{A}})$ converges in $\widetilde{\mathbb{A}_{P}}$ and we have the equality in $\mathbb{T}_{n, z}\left(K_{P}\right)$ :

$$
\mathcal{L}_{P}(\widetilde{\varphi} / \widetilde{\mathbb{A}})=\frac{\left[\widetilde{\varphi}\left(\frac{\widetilde{\mathbb{A}}}{P \widetilde{\mathbb{A}}}\right)\right]_{\widetilde{\mathbb{A}}}}{P} \log _{\widetilde{\varphi}, P}\left(u_{\phi}\left(t_{1}, \ldots, t_{n} ; z\right)\right) .
$$

Proof. Let $\rho: A \rightarrow A\{\tau\}$ be the Drinfeld $A$-module given by:

$$
\rho_{\theta}=\sum_{j=0}^{r} \alpha_{j} P^{q^{j}-1} \tau^{j}
$$

where $\phi_{\theta}=\sum_{j=0}^{r} \alpha_{j} \tau^{j}$. Let $\varrho$ be the canonical deformation of $\rho$ over $\mathbb{T}_{n}\left(K_{\infty}\right)$ and $\widetilde{\varrho}$ be the canonical $z$-deformation of $\varrho$. Then:

$$
\log _{\widetilde{\varrho}}=\sum_{j \geq 0} b_{j}\left(t_{1}\right) \cdots b_{j}\left(t_{n}\right) P^{q^{j}-1} l_{j} z^{j} \tau^{j},
$$

where $\log _{\phi}=\sum_{j \geq 0} l_{i} \tau^{i}$. By (18), we have the formal identity in $K\left(t_{1}, \ldots, t_{n}\right)[[z]]$ :

$$
\mathcal{L}(\widetilde{\varrho} / \widetilde{\mathbb{A}})=\log _{\widetilde{\varrho}}\left(u_{\rho}\left(t_{1}, \ldots, t_{n} ; z\right)\right) .
$$

But, by Lemma 3.20, we have:

$$
\forall j \geq 0, v_{P}\left(b_{j}\left(t_{1}\right) \cdots b_{j}\left(t_{n}\right) P^{q^{j}-1} l_{j}\right) \geq q^{j}-1-\left[\frac{j}{d}\right] .
$$

This implies that $\mathcal{L}(\widetilde{\varrho} / \widetilde{\mathbb{A}})$ converges in $\widetilde{\mathbb{A}_{P}}$. Let $Q$ be a monic irreducible element in $A$ prime to $P$. The multiplication by $P$ gives rise to an isomorphism of $\widetilde{\mathbb{A}}$-modules:

$$
\widetilde{\varrho}\left(\frac{\widetilde{\mathbb{A}}}{Q \widetilde{\mathbb{A}}}\right) \simeq \widetilde{\varphi}\left(\frac{\widetilde{\mathbb{A}}}{Q \widetilde{\mathbb{A}}}\right)
$$

This implies that we have the following equality in $K\left(t_{1}, \ldots, t_{n}\right)[[z]]$ :

$$
\mathcal{L}(\widetilde{\varrho} / \widetilde{\mathbb{A}})=\mathcal{L}_{P}(\widetilde{\varphi} / \widetilde{\mathbb{A}}) .
$$

Therefore we get the first assertion of the Theorem. The second assertion is a consequence of (8) and Lemma 3.22 
Observe that we have analogous constructions for $\varphi$ as that made in Lemma 3.21 and Lemma 3.22, Thus, we have a continuous morphism of $\mathbb{F}_{q}\left[t_{1}, \ldots, t_{n}\right]$-modules:

$$
\log _{\varphi, P}: \mathbb{O} \rightarrow \mathbb{T}_{n}\left(\mathbb{C}_{P}\right)
$$

such that:

$$
\forall a \in \mathbb{A}, \log _{\varphi, P}\left(\varphi_{a}(x)\right)=a \log _{\varphi, P}(x) .
$$

But, $\log _{\varphi, P}$ is no longer injective.

Let ev : $\mathbb{T}_{n, z}\left(\mathbb{C}_{P}\right) \rightarrow \mathbb{T}_{n}\left(\mathbb{C}_{P}\right),\left.y \mapsto y\right|_{z=1}$. Then:

$$
\forall y \in \widetilde{\mathbb{O}}, e v\left(\log _{\widetilde{\varphi}, P}(y)\right)=\log _{\varphi, P}(e v(y)) .
$$

We set:

$$
\mathcal{L}_{P}(\varphi / \mathbb{A})=\operatorname{ev}\left(\mathcal{L}_{P}(\widetilde{\varphi} / \widetilde{\mathbb{A}})\right) \in \mathbb{A}_{P}
$$

We get:

Corollary 3.24. We have the following equality in $\mathbb{T}_{n}\left(K_{P}\right)$ :

$$
\mathcal{L}_{P}(\varphi / \mathbb{A})=\frac{\left[\varphi\left(\frac{\mathbb{A}}{P \mathbb{A}}\right)\right]_{\mathbb{A}}}{P} \log _{\varphi, P}\left(u_{\phi}\left(t_{1}, \ldots, t_{n} ; 1\right)\right) .
$$

In particular $\mathcal{L}_{P}(\varphi / \mathbb{A})=0$ if and only if $u_{\phi}\left(t_{1}, \ldots, t_{n} ; 1\right)$ is a torsion point for $\varphi$.

Proof. This is a direct consequence of Theorem 3.23 ,

We set:

$$
\begin{gathered}
\mathcal{L}_{P}^{(1)}(\varphi / \mathbb{A})=\operatorname{ev}\left(\frac{d}{d z} \mathcal{L}_{P}(\widetilde{\varphi} / \widetilde{\mathbb{A}})\right) \in \mathbb{A}_{P}, \\
u_{\phi}^{(1)}\left(t_{1}, \ldots, t_{n} ; 1\right)=\operatorname{ev}\left(\frac{d}{d z} u_{\phi}\left(t_{1}, \ldots, t_{n} ; z\right)\right) \in A\left[t_{1}, \ldots, t_{n}\right], \\
\forall a \in A\left[t_{1}, \ldots, t_{n}\right], \varphi_{a}^{(1)}=\operatorname{ev}\left(\frac{d}{d z} \widetilde{\varphi}_{a}\right) \in A\left[t_{1}, \ldots, t_{n}\right]\{\tau\} .
\end{gathered}
$$

Proposition 3.25. Let's assume that $u_{\phi}\left(t_{1}, \ldots, t_{n} ; 1\right)$ is a torsion point for $\varphi$. Then, in $\mathbb{T}_{n}\left(K_{P}\right), \mathcal{L}_{P}^{(1)}(\varphi / \mathbb{A})$ is equal to

$$
\frac{\left[\varphi\left(\frac{\mathbb{A}}{P \mathbb{A}}\right)\right]_{\mathbb{A}}}{P} \log _{\varphi, P}\left(u_{\phi}^{(1)}\left(t_{1}, \ldots, t_{n} ; 1\right)\right)+\frac{\left[\varphi\left(\frac{\mathbb{A}}{P \mathbb{A}}\right)\right]_{\mathbb{A}}}{a P} \log _{\varphi, P}\left(\varphi_{a}^{(1)}\left(u_{\phi}\left(t_{1}, \ldots, t_{n} ; 1\right)\right)\right),
$$

for any $a \in A\left[t_{1}, \ldots, t_{n}\right]$ which is monic as a polynomial in $\theta$ and such that $\varphi_{a}\left(u_{\phi}\left(t_{1}, \ldots, t_{n} ; 1\right)\right)=0$.

Proof. Let's start with the equality in $K\left(t_{1}, \ldots, t_{n}\right)[[z]]$ :

$$
\mathcal{L}(\widetilde{\varphi} / \widetilde{\mathbb{A}})=\log _{\widetilde{\varphi}}\left(u_{\phi}\left(t_{1}, \ldots, t_{n} ; z\right)\right)
$$

We get, for $a \in A\left[t_{1}, \ldots t_{n}\right]$ :

$$
a \mathcal{L}(\widetilde{\varphi} / \widetilde{\mathbb{A}})=\log _{\widetilde{\varphi}}\left(\widetilde{\varphi}_{a}\left(u_{\phi}\left(t_{1}, \ldots, t_{n} ; z\right)\right)\right)
$$

Thus

$$
\begin{aligned}
a \frac{d}{d z} \mathcal{L}(\widetilde{\varphi} / \widetilde{\mathbb{A}})= & \left(\frac{d}{d z} \log _{\widetilde{\varphi}}\right)\left(\widetilde{\varphi}_{a}\left(u_{\phi}\left(t_{1}, \ldots, t_{n} ; z\right)\right)\right)+\log _{\widetilde{\varphi}}\left(\frac{d}{d z}\left(\widetilde{\varphi}_{a}\right)\left(u_{\phi}\left(t_{1}, \ldots, t_{n} ; z\right)\right)\right) \\
& +\log _{\widetilde{\varphi}}\left(\widetilde{\varphi}_{a}\left(\frac{d}{d z}\left(u_{\phi}\left(t_{1}, \ldots, t_{n} ; z\right)\right)\right)\right) .
\end{aligned}
$$

Now, by Remark 3.3 , there exists an element $a \in A\left[t_{1}, \ldots, t_{n}\right]$ which is monic as a polynomial in $\theta$ such that:

$$
\varphi_{a}\left(u_{\phi}\left(t_{1}, \ldots, t_{n} ; z\right)\right)=0
$$


The assertion of the Proposition follows from Corollary 3.24 and Theorem 3.23 ,

\section{Arithmetic of CyClotomic FunCtion fields}

Let $p$ be an odd prime number and let $X$ be the $p$-Sylow subgroup of the ideal class group of $\mathbb{Q}\left(e^{\frac{2 i \pi}{p}}\right)$. Let $\Delta=\operatorname{Gal}\left(\mathbb{Q}\left(e^{\frac{2 i \pi}{p}}\right) / \mathbb{Q}\right) \simeq\left(\frac{\mathbb{Z}}{p \mathbb{Z}}\right)^{\times}$and let $\widehat{\Delta}=\operatorname{Hom}\left(\Delta, \mathbb{Z}_{p}^{\times}\right)$. Observe that $X$ is a $\mathbb{Z}_{p}[\Delta]$-module. For $\chi \in \widehat{\Delta}$, let:

$$
X(\chi)=e_{\chi} X
$$

where $e_{\chi}=\frac{1}{|\Delta|} \sum_{\delta \in \Delta} \chi(\delta) \delta^{-1} \in \mathbb{Z}_{p}[\Delta]$.

Let $\omega_{p} \in \widehat{\Delta}$ be the $p$-adic Teichmüller character. Let $\chi \in \widehat{\Delta}$ be an odd character. Then by the famous Leopoldt's Spiegelungssatz ([20]), we have:

$$
\operatorname{dim}_{\mathbb{F}_{p}} \frac{X\left(\omega_{p} \chi^{-1}\right)}{p X\left(\omega_{p} \chi^{-1}\right)} \leq \operatorname{dim}_{\mathbb{F}_{p}} \frac{X(\chi)}{p X(\chi)} \leq \operatorname{dim}_{\mathbb{F}_{p}} \frac{X\left(\omega_{p} \chi^{-1}\right)}{p X\left(\omega_{p} \chi^{-1}\right)}+1 .
$$

Therefore if $X\left(\omega_{p} \chi^{-1}\right)=\{0\}, X(\chi)$ is a cyclic $\mathbb{Z}_{p}$-module. In particular, if Vandiver's Conjecture is true for the prime $p$ then $X(\chi)$ is a cyclic $\mathbb{Z}_{p}$-module for every odd character $\chi$ of conductor $p$. The cyclicity of $X$ as a $\mathbb{Z}_{p}[\Delta]$-module is still an open problem and is conjectured to be true. This problem is sometimes referred as "The Iwasawa-Leopoldt Conjecture" ([19], page 80), and by the above discussion Vandiver's Conjecture implies this cyclicity statement.

In this section, using the ideas developed in sections 2 and 3 we study an analogous question for the case of the $P$ th cyclotomic function field where $P$ is a monic irreducible element in $A$. Note that the analogue of Vandiver's Conjecture is false in this context $(7)$. Furthermore, we are not convinced that the isotypic components of the "P-Sylow subgroup " of Taelman's class module associated to the Pth cyclotomic function field are cyclic $A_{P}$-modules, where $A_{P}$ is the $P$-adic completion of $A$, even in the case of odd characters (it would be very interesting to exhibit, if they exist, such examples). However we are able to prove a cyclicity result (Theorem 4.3) that involves the "derivatives" of Goss $P$-adic $L$-series for odd characters. This result does not seem to have an analogous counterpart in the classical case.

\subsection{Dirichlet characters.}

Let $\overline{\mathbb{F}_{q}}$ be the algebraic closure of $\mathbb{F}_{q}$ in $\mathbb{C}_{\infty}$. Let $\zeta \in \overline{\mathbb{F}_{q}}$ and let $Q$ be the monic irreducible element in $A$ such that $Q(\zeta)=0$. The morphism of $\mathbb{F}_{q}$-algebras $\rho_{\zeta}: A \rightarrow \mathbb{C}_{\infty}, a \mapsto a(\zeta)$, induces an injective morphism of groups still denoted by $\rho_{\zeta}:$

$$
\rho_{\zeta}:\left(\frac{A}{Q A}\right)^{\times} \rightarrow \mathbb{C}_{\infty}^{\times} .
$$

A Dirichlet character is a morphism of groups $\chi:\left(\frac{A}{g A}\right)^{\times} \rightarrow \mathbb{C}_{\infty}^{\times}$, for some $g \in A_{+}$. We observe that the conductor of $\chi, f_{\chi} \in A_{+}$, is a square-free monic polynomial. In this article all Dirichlet characters are assumed to be primitive, i.e. viewed as defined modulo their conductors. For example, if $\zeta \in \overline{\mathbb{F}_{q}}, \rho_{\zeta}$ is a Dirichlet character of conductor $Q$, where $Q$ is the monic irreducible polynomial in $A$ such that $Q(\zeta)=0$. 
Let $\chi$ be a (primitive) Dirichlet character. Then there exist $\zeta_{1}(\chi), \ldots, \zeta_{m}(\chi) \in$ $\overline{\mathbb{F}_{q}}, n_{1}(\chi), \ldots, n_{m}(\chi) \in\{1, \ldots, q-1\}, m \geq 0$, such that:

$$
\forall a \in A, \chi(a)=\prod_{j=1}^{m} a\left(\zeta_{j}(\chi)\right)^{n_{j}(\chi)} .
$$

Note that the conductor of $\chi$ is the least common multiple of the conductors of the $\rho_{\zeta_{j}(\chi)}, j=1, \ldots, m$. The type of $\chi, s(\chi)$, is defined by:

$$
s(\chi)=\sum_{j=1}^{m} n_{j}(\chi) .
$$

For example, the only Dirichlet character of type 0 is the trivial character, and the Dirichlet characters of type 1 are exactly the $\rho_{\zeta}, \zeta \in \overline{\mathbb{F}_{q}}$. A Dirichlet character, $\chi$, is called odd if $s(\chi) \equiv 1(\bmod q-1)$, and even otherwise.

Let's select $\lambda_{\theta} \in \mathbb{C}_{\infty}$ such that $\lambda_{\theta}^{q-1}=-\theta$. We set:

$$
\widetilde{\pi}=\lambda_{\theta} \theta \prod_{j \geq 1}\left(1-\theta^{1-q^{j}}\right)^{-1} \in K_{\infty}\left(\lambda_{\theta}\right)^{\times} .
$$

Recall that $\tau: \mathbb{C}_{\infty} \rightarrow \mathbb{C}_{\infty}$ is the continuous morphism of $\mathbb{F}_{q}$-algebras such that $\tau(\theta)=\theta^{q}$. Let $C$ be the Carlitz module (recall that $C_{\theta}=\tau+\theta$ ). Then:

$$
\operatorname{Ker}\left(\exp _{C}: \mathbb{C}_{\infty} \rightarrow \mathbb{C}_{\infty}\right)=\tilde{\pi} A \text {. }
$$

Observe that:

$$
\lambda_{\theta}=\exp _{C}\left(\frac{\tilde{\pi}}{\theta}\right) .
$$

For $a \in A_{+}$, we set: $\lambda_{a}=\exp _{C}\left(\frac{\widetilde{\pi}}{a}\right) \in \mathbb{F}_{q}\left(\left(\frac{1}{\lambda_{\theta}}\right)\right)$. Let $\sigma: \overline{\mathbb{F}_{q}}\left(\left(\frac{1}{\lambda_{\theta}}\right)\right) \rightarrow \overline{\mathbb{F}_{q}}\left(\left(\frac{1}{\lambda_{\theta}}\right)\right)$ be the continuous morphism of $\overline{\mathbb{F}_{q}}$-algebras such that $\sigma\left(\lambda_{\theta}\right)=\lambda_{\theta}^{q}$.

Finally, we recall the definition of the Gauss-Thakur sum attached to a Dirichlet character. Let $\zeta \in \overline{\mathbb{F}_{q}}$ and let $Q$ be the monic irreducible element in $A$ such that $Q(\zeta)=0$. We set (see also [29]):

$$
g\left(\rho_{\zeta}\right)=-\sum_{n=0}^{\operatorname{deg}_{\theta} Q-1} \sum_{a \in A_{+, n}} \rho_{\zeta}(a)^{-1} C_{a}\left(\lambda_{Q}\right) \in \overline{\mathbb{F}_{q}}\left(\left(\frac{1}{\lambda_{\theta}}\right)\right)^{\times} .
$$

One easily verifies that:

$$
\sigma\left(g\left(\rho_{\zeta}\right)\right)=(\zeta-\theta) g\left(\rho_{\zeta}\right)
$$

In particular:

$$
\tau^{\operatorname{deg}_{\theta} Q}\left(g\left(\rho_{\zeta}\right)\right)=\sigma^{\operatorname{deg}_{\theta} Q}\left(g\left(\rho_{\zeta}\right)\right)=(-1)^{\operatorname{deg}_{\theta} Q} Q g\left(\rho_{\zeta}\right) .
$$

Also, we have (29], Proposition I ):

$$
\sum_{k=0}^{\operatorname{deg}_{\theta} Q-1} g\left(\rho_{\zeta^{q^{k}}}\right)=\lambda_{Q}
$$

Now, let $\chi$ be a Dirichlet character. Let $\zeta_{1}(\chi), \ldots, \zeta_{m}(\chi) \in \overline{\mathbb{F}_{q}}, n_{1}(\chi), \ldots, n_{m}(\chi) \in$ $\{1, \ldots, q-1\}, m \geq 0$, such that: $\forall a \in A, \chi(a)=\prod_{j=1}^{m} a\left(\zeta_{j}(\chi)\right)^{n_{j}(\chi)}$. We set (see 
also [4]):

$$
g(\chi)=\prod_{j=1}^{m} g\left(\rho_{\zeta_{j}(\chi)}\right)^{n_{j}(\chi)}
$$

Then:

$$
\sigma(g(\chi))=\prod_{j=1}^{m}\left(\zeta_{j}(\chi)-\theta\right)^{n_{j}(\chi)} g(\chi) .
$$

\section{2. $P$-adic Dirichlet-Goss $L$-series.}

Let $P$ be a monic irreducible polynomial in $A$ of degree $d$. Let $K^{a c}$ be the algebraic closure of $K$ in $\mathbb{C}_{\infty}$ and we fix once for all a $K$-embedding of $K^{a c}$ in $\mathbb{C}_{P}$. Let $n \geq 0$ be an integer and let $t_{1}, \ldots, t_{n}, z$ be $n+1$ indeterminates over $\mathbb{C}_{P}$. Let's set:

$$
L_{P}\left(t_{1}, \ldots, t_{n} ; z\right)=\sum_{n \geq 0} \sum_{a \in A_{+, n}, a \neq 0} \frac{a\left(t_{1}\right) \ldots a\left(t_{n}\right)}{a} z^{n} \in K\left[t_{1}, \ldots, t_{n}\right][[z]] .
$$

Then by Theorem 3.23 ,

$$
L_{P}\left(t_{1}, \ldots, t_{n} ; z\right) \in \mathbb{T}_{n, z}\left(K_{P}\right) .
$$

Let $\chi$ be a Dirichlet character. Let $\zeta_{1}(\chi), \ldots, \zeta_{m}(\chi) \in \overline{\mathbb{F}_{q}}, n_{1}(\chi), \ldots, n_{m}(\chi) \in$ $\{1, \ldots, q-1\}, m \geq 0$, such that: $\forall a \in A, \chi(a)=\prod_{j=1}^{m} a\left(\zeta_{j}(\chi)\right)^{n_{j}(\chi)}$. Let $n=$ $n_{1}(\chi)+\cdots+n_{m}(\chi)$ be the type of $\chi$. Let's recall that the value at one of the Dirichlet-Goss $P$-adic $L$-series attached to $\chi$ is defined by (see [15]):

$$
L_{P}(1, \chi)=\sum_{n \geq 0} \sum_{a \in A_{+, n}, a \neq 0} \frac{\chi(a)}{a} \in \mathbb{C}_{P} .
$$

Now let $\eta_{1}, \ldots, \eta_{n} \in \overline{\mathbb{F}_{q}}$ such that $\eta_{n_{1}(\chi)+\cdots n_{j-1}(\chi)+k}=\zeta_{j}$, for $k \in\left\{1, \ldots, n_{j}(\chi)\right\}$, $j=1, \ldots, m$. Let $e v_{\chi}: K\left[t_{1}, \ldots, t_{n}\right][[z]] \rightarrow \overline{\mathbb{F}_{q}}(\theta)[[z]]$, be the $K$-linear map defined by:

$$
e v_{\chi}(f)=\left.f\right|_{t_{1}=\zeta_{1}, \ldots, t_{n}=\zeta_{n}} .
$$

It is clear that we have:

$$
L_{P}(1, \chi)=\left.e v_{\chi}\left(L_{P}\left(t_{1}, \ldots, t_{n} ; z\right)\right)\right|_{z=1} .
$$

This implies that, by Corollary 3.24 and Lemma 3.7 and Lemma 3.9, if $\chi$ is odd then:

$$
L_{P}(1, \chi)=0
$$

Thus, if $\chi$ is odd, we set:

$$
L_{P}^{(1)}(1, \chi)=\frac{d}{d z}\left(\left.e v_{\chi}\left(L_{P}\left(t_{1}, \ldots, t_{n} ; z\right)\right)\right|_{z=1} \in \mathbb{C}_{P} .\right.
$$

Theorem 4.1. Let $\chi$ be a Dirichlet character. Then $L_{P}(1, \chi)=0$ if and only if $\chi$ is odd. Furthermore, if $\chi$ is odd, we have:

$$
L_{P}^{(1)}(1, \chi) \neq 0 .
$$


Note that the case of characters of conductor $P$ and $\chi$ even is treated in [7] and the non-vanishing result uses Bosser's P-adic Baker Brumer Theorem (see the appendix of [7]). In what follows, we propose a new approach which does not use the $P$-adic Baker-Brumer Theorem.

We will now work in $K\left[t_{1}, \ldots, t_{n}\right][[z]]$. Let

$$
\tau: K\left[t_{1}, \ldots, t_{n}\right][[z]] \rightarrow K\left[t_{1}, \ldots, t_{n}\right][[z]]
$$

be the continuous morphism (for the $z$-adic topology) of $\mathbb{F}_{q}\left[t_{1}, \ldots, t_{n}\right][[z]]$-algebras such that

$$
\forall x \in K, \tau(x)=x^{q}
$$

Set :

$$
\log _{n, z}=\sum_{k \geq 0} \frac{b_{i}\left(t_{1}\right) \cdots b_{i}\left(t_{n}\right)}{\ell_{k}} z^{k} \tau^{k}
$$

where for $j=1, \ldots, n$, we recall that $b_{0}\left(t_{j}\right)=1$ and for $i \geq 1: b_{i}\left(t_{j}\right)=\prod_{k=0}^{i-1}\left(t_{j}-\right.$ $\left.\theta^{q^{k}}\right), \ell_{0}=1$ and for $n \geq 1, \ell_{n}=\left(\theta-\theta^{q^{n}}\right) \ell_{n-1}$. Then, by (11), there exists $u_{C}\left(t_{1}, \ldots, t_{n} ; z\right) \in A\left[t_{1}, \ldots, t_{n}, z\right]$ such that we have in $K\left[t_{1}, \ldots, t_{n}\right][[z]]$ :

$$
\sum_{k \geq 0} \sum_{a \in A_{+, k}} \frac{a\left(t_{1}\right) \cdots a\left(t_{n}\right)}{a} z^{k}=\log _{n, z}\left(u_{C}\left(t_{1}, \ldots, t_{n} ; z\right)\right) .
$$

Let $\chi$ be a Dirichlet character of type $n$ and conductor $f$. Recall that, by (14), there exists $\eta_{1}, \ldots, \eta_{n} \in \overline{\mathbb{F}_{q}}$ such that :

$$
\sigma(g(\chi))=\left(\eta_{1}-\theta\right) \cdots\left(\eta_{n}-\theta\right) g(\chi) .
$$

We set :

$$
u_{\chi}(z)=\left.g(\chi) u_{C}\left(t_{1}, \ldots, t_{n} ; z\right)\right|_{t_{1}=\eta_{1}, \ldots, t_{n}=\eta_{n}} \in g(\chi) \mathbb{F}_{q}\left(\eta_{1}, \ldots, \eta_{n}\right)[\theta][z] .
$$

Let $[\chi]=\left\{\chi^{q^{i}}, i \geq 0\right\}$. Observe that for $\psi \in[\chi], \psi$ is of type $n$ and conductor $f$. We set :

$$
u_{[\chi]}(z)=\sum_{\psi \in[\chi]} u_{\psi}(z) \in A\left[\lambda_{f}\right][z]
$$

and :

$$
u_{[\chi]}^{(1)}(z)=\frac{d}{d z} u_{[\chi]}(z) .
$$

Finally, let $\Delta_{f}=\operatorname{Gal}\left(K\left(\lambda_{f}\right) / K\right)$.

Let $\log _{C, P}: O \rightarrow \mathbb{C}_{P}$ be the $P$-adic logarithm for the Carlitz module introduced in section 3.4, where $O=\left\{x \in \mathbb{C}_{P}, v_{P}(x) \geq 0\right\}$.

Proposition 4.2. Let $\chi$ be a Dirichlet character of type $n$ and conductor $f$. 1) If $n \not \equiv 1(\bmod q-1)$, then:

$$
L_{P}(1, \chi) g(\chi)=\left(1-P^{-1} \chi(P)\right) \frac{1}{\left|\Delta_{f}\right|} \sum_{\mu \in \Delta_{f}} \chi^{-1}(\mu) \log _{C, P}\left(\mu\left(u_{[\chi]}(1)\right)\right) .
$$

2) If $n \geq q, n \equiv 1(\bmod q-1)$, then :

$$
L_{P}^{(1)}(1, \chi) g(\chi)=\left(1-P^{-1} \chi(P)\right) \frac{1}{\left|\Delta_{f}\right|} \sum_{\mu \in \Delta_{f}} \chi^{-1}(\mu) \log _{C, P}\left(\mu\left(u_{[\chi]}^{(1)}(1)\right)\right) .
$$


3) If $n=1$, we have:

$$
L_{P}^{(1)}(1, \chi) g(\chi)=\left(1-P^{-1} \chi(P)\right) \frac{1}{\left|\Delta_{f}\right|} \frac{1}{\theta-\chi(\theta)} \sum_{\mu \in \Delta_{f}} \chi^{-1}(\mu) \log _{C, P}\left(\mu\left(\lambda_{f}^{q}\right)\right) .
$$

Proof. Let's set :

$$
\log _{\sigma, z}=\sum_{k \geq 0} \frac{1}{\ell_{k}} z^{k} \sigma^{k}
$$

If we specialize $t_{i}$ in $\eta_{i}$ in the formula (15) and multiply by $g(\chi)$, we have the following equality in $\mathbb{F}_{q}\left(\eta_{1}, \ldots, \eta_{n}\right)[\theta]\left[\lambda_{f}\right][[z]]$ :

$$
\sum_{k \geq 0} \sum_{a \in A_{+, k}} \frac{\chi(a)}{a} g(\chi) z^{k}=\log _{\sigma, z} u_{\chi}(z) .
$$

Now, set :

$$
\log _{C, z}=\sum_{k \geq 0} \frac{1}{\ell_{k}} z^{k} \tau^{k}
$$

We get the following equality in $K\left[\lambda_{f}\right][[z]]$ ( $\tau$ acts trivially on $z$ ):

$$
\sum_{\psi \in[\chi]} \sum_{k \geq 0} \sum_{a \in A_{+, k}} \frac{\psi(a)}{a} g(\psi) z^{k}=\log _{C, z} u_{[\chi]}(z)
$$

For $\mu \in \Delta_{f}$, we have : $\mu(g(\chi))=\chi(\mu) g(\chi)$, we therefore deduce that :

$$
\left|\Delta_{f}\right| \sum_{k \geq 0} \sum_{a \in A_{+, k}} \frac{\chi(a)}{a} g(\chi) z^{k}=\sum_{\mu \in \Delta_{f}} \chi^{-1}(\mu) \log _{C, z} \mu\left(u_{[\chi]}(z)\right) .
$$

Thus:

$$
\left|\Delta_{f}\right| g(\chi) \sum_{k \geq 0} \sum_{a \in A_{+, k}} \frac{\chi(a)}{a} z^{k}=\sum_{\mu \in \Delta_{f}} \chi^{-1}(\mu) \log _{C, z} \mu\left(u_{[\chi]}(z)\right) .
$$

And finally :

$g(\chi) \sum_{k \geq 0} \sum_{\substack{a \in A_{+, k} \\ a \neq 0 \\ \bmod P}} \frac{\chi(a)}{a} z^{k}=\left(1-P^{-1} \chi(P) z^{d}\right) \frac{1}{\left|\Delta_{f}\right|} \sum_{\mu \in \Delta_{f}} \chi^{-1}(\mu) \log _{C, z} \mu\left(u_{[\chi]}(z)\right)$.

Recall that :

$$
\theta \log _{C, z}=\log _{C, z}(z \tau+\theta) .
$$

Now, since $u_{[\chi]}(1)$ can be a $P$-adic unit, using the functional equation of $\log _{C, z}$ if necessary, we get if $\chi$ is even (i.e. $n \not \equiv 1(\bmod q-1))$ :

$$
L_{P}(1, \chi) g(\chi)=\left(1-P^{-1} \chi(P)\right) \frac{1}{\left|\Delta_{f}\right|} \sum_{\mu \in \Delta_{f}} \chi^{-1}(\mu) \log _{C, P}\left(\mu\left(u_{[\chi]}(1)\right)\right) .
$$

Let's treat the case where $\chi$ is odd (i.e. $n \equiv 1(\bmod q-1))$. If $n \geq q$, by Lemma 3.9. we have $u_{[\chi]}(1)=0$. Using again the same technique, we get in this case:

$$
L_{P}^{(1)}(1, \chi) g(\chi)=\left(1-P^{-1} \chi(P)\right) \frac{1}{\left|\Delta_{f}\right|} \sum_{\mu \in \Delta_{f}} \chi^{-1}(\mu) \log _{C, P}\left(\mu\left(u_{[\chi]}^{(1)}(1)\right)\right) .
$$

Thus, it remains to treat the case $n=1$. First, by formula (13) and Lemma 3.7 ,

$$
u_{[\chi]}(z)=\lambda_{f}
$$


BRUNO ANGLÈS AND FLORIC TAVARES RIBEIRO

Therefore, in this case :

$$
g(\chi) \sum_{k \geq 0} \sum_{\substack{a \in A_{+, k} \\ a \neq 0}} \frac{\chi(a)}{a} z^{k}=\left(1-P^{-1} \chi(P) z^{d}\right) \frac{1}{\left|\Delta_{f}\right|} \sum_{\mu \in \Delta_{f}} \chi^{-1}(\mu) \log _{C, z} \mu\left(\lambda_{f}\right) .
$$

Now, select $\lambda \in \mathbb{F}_{q}$ such that $f$ is relatively prime to $\theta+\lambda$. Then :

$$
\sum_{\mu \in \Delta_{f}} \chi^{-1}(\mu) \log _{C, z} \mu\left(\lambda_{f}\right)=\chi^{-1}(\theta+\lambda) \sum_{\mu \in \Delta_{f}} \chi^{-1}(\mu) \log _{C, z} C_{\theta+\lambda}\left(\mu\left(\lambda_{f}\right)\right) .
$$

Thus $g(\chi)(\chi(\theta+\lambda)-(\theta+\lambda)) \sum_{k \geq 0} \sum_{a \in A_{+, k}, a \neq 0(\bmod P)} \frac{\chi(a)}{a} z^{k}$ is equal to:

$$
\left(1-P^{-1} \chi(P) z^{d}\right) \frac{1}{\left|\Delta_{f}\right|} \sum_{\mu \in \Delta_{f}} \chi^{-1}(\mu)\left(\log _{C, z} C_{\theta+\lambda}\left(\mu\left(\lambda_{f}\right)\right)-(\theta+\lambda) \log _{C, z} \mu\left(\lambda_{f}\right)\right) .
$$

But $n=1$, thus $\chi(\theta+\lambda)=\chi(\theta)+\lambda$. Thus,

$$
g(\chi)(\chi(\theta)-\theta) \sum_{k \geq 0} \sum_{a \in A_{+, k}, a \neq 0} \frac{\chi(a)}{a} z^{k}
$$

is equal to :

$$
\left(1-P^{-1} \chi(P) z^{d}\right) \frac{1}{\left|\Delta_{f}\right|} \sum_{\mu \in \Delta_{f}} \chi^{-1}(\mu)\left(\log _{C, z} C_{\theta}\left(\mu\left(\lambda_{f}\right)\right)-\theta \log _{C, z} \mu\left(\lambda_{f}\right)\right) .
$$

Set :

$$
\log _{C, z}^{(1)}=\sum_{k \geq 0} \frac{k}{\ell_{k}} z^{k-1} \tau^{k}
$$

Then :

$$
\log _{C, z}^{(1)}(z \tau+\theta)-\theta \log _{C, z}^{(1)}=-\log _{C, z} \tau .
$$

Therefore, for $n=1$, we get :

$$
g(\chi) L_{P}^{(1)}(1, \chi)=\left(1-P^{-1} \chi(P)\right) \frac{1}{\left|\Delta_{f}\right|} \frac{1}{\theta-\chi(\theta)} \sum_{\mu \in \Delta_{f}} \chi^{-1}(\mu) \log _{C, P}\left(\mu\left(\lambda_{f}^{q}\right)\right) .
$$

Proof of Theorem 4.1:

We first treat the case where $\chi$ is even (i.e. $n \not \equiv 1(\bmod q-1))$. Note that by Proposition 3.8 and Lemma 3.7, we have :

$$
u_{[\chi]}(1) \neq 0 .
$$

Since $\chi$ is even this implies that $u_{[\chi]}(1)$ is not a torsion point for the Carlitz module, in particular :

$$
\log _{C, P}\left(u_{[\chi]}(1)\right) \neq 0 \text {. }
$$

But, by the proof of Proposition 4.2, we have :

$$
\log _{C, P}\left(u_{[\chi]}(1)\right)=\sum_{\psi \in[\chi]}\left(1-\frac{\psi(P)}{P}\right)^{-1} L_{P}(1, \psi) g(\psi) .
$$

This implies that there exists $\psi \in[\chi]$ such that :

$$
L_{P}(1, \psi) \neq 0 \text {. }
$$


Thus, we have to prove that if $L_{P}(1, \chi) \neq 0$, then $L_{P}\left(1, \chi^{\frac{1}{q}}\right) \neq 0$. We are going to prove it by performing a change of variable.

Set $K^{(q)}=\mathbb{F}_{q}\left(\theta^{q}\right)$, observe that $K / K^{(q)}$ is totally ramified at every place of $K^{(q)}$. Let $C^{(q)}$ be the Carlitz module for $A^{(q)}:=A^{q}=\mathbb{F}_{q}\left[\theta^{q}\right]$, i.e. $C^{(q)}{ }_{\theta^{q}}=\tau+\theta^{q}$. Let $\lambda_{f\left(\theta^{q}\right)}^{(q)}=\exp _{C^{(q)}}\left(\frac{\tilde{\pi}^{(q)}}{f\left(\theta^{q}\right)}\right)$ (this is also equal to $\left.\lambda_{f}^{q}\right)$. Then :

$$
K\left(\lambda_{f}\right)=K\left(\lambda_{f\left(\theta^{q}\right)}^{(q)}\right) .
$$

Furthermore, the restriction map induces an isomorphism of groups :

$$
\Delta_{f} \simeq \Delta_{f\left(\theta^{q}\right)}^{(q)} .
$$

This implies that :

$$
\forall \sigma \in \Delta_{f}, \chi(\sigma)=\chi\left(\sigma^{(q)}\right)
$$

Let $\left(., K\left(\lambda_{f}\right) / K\right)$ be the Artin map, then for a monic irreducible element $Q$ of $A$ prime to $f$ :

$$
\left.\left(Q, K\left(\lambda_{f}\right) / K\right)\right|_{K^{(q)}\left(\lambda_{f\left(\theta^{q}\right)}^{(q)}\right)}=\left(Q\left(\theta^{q}\right), K^{(q)}\left(\lambda_{f\left(\theta^{q}\right)}^{(q)}\right) / K^{(q)}\right) .
$$

Observe that:

$$
L_{P}(1, \chi)=\sum_{n \geq 0} \sum_{a \in A_{+, n}, a \neq 0} \quad \frac{\chi\left(\left(a, K\left(\lambda_{f}\right) / K\right)\right)}{a} .
$$

Therefore $L_{P}(1, \chi) \neq 0$ implies that $L_{P}^{(q)}(1, \chi) \neq 0$, where :

$$
L_{P}^{(q)}(1, \chi)=\sum_{n \geq 0} \sum_{b \in A_{+, n}^{(q)}, b \neq 00} \frac{\chi\left(\left(b, K^{(q)}\left(\lambda_{f\left(\theta^{q}\right)}^{(q)}\right) / K^{(q)}\right)\right)}{b} .
$$

But :

$$
L_{P}^{(q)}(1, \chi)=\left(L_{P}\left(1, \chi^{\frac{1}{q}}\right)\right)^{q} .
$$

Thus:

$$
L_{P}\left(1, \chi^{\frac{1}{q}}\right) \neq 0 .
$$

Now, we turn to the case where $\chi$ is odd of type $n \geq q$. Again, by Proposition 3.8 , we get :

$$
u_{[\chi]}^{(1)}(1) \neq 0 .
$$

Since $\chi$ is not of type 1 , by formula (13),$u_{[\chi]}^{(1)}(1)$ is not a torsion point, in particular:

$$
\log _{C, P}\left(u_{[\chi]}^{(1)}(1)\right) \neq 0 .
$$

Now by the proof of Theorem 4.2 ;

$$
\log _{C, P}\left(u_{[\chi]}^{(1)}(1)\right)=\sum_{\psi \in[\chi]}\left(1-\frac{\psi(P)}{P}\right)^{-1} L_{P}^{(1)}(1, \psi) g(\psi) .
$$

We can conclude as in the even case. It remains to treat the case $n=1$. Observe that $\lambda_{f}^{q}$ is not a torsion point for the Carlitz module. Furthermore by Proposition 4.2, we have :

$$
\log _{C, P}\left(\lambda_{f}^{q}\right)=\sum_{\psi \in[\chi]}(\theta-\psi(\theta))\left(1-\frac{\psi(P)}{P}\right)^{-1} L_{P}^{(1)}(1, \psi) g(\psi) .
$$


Again, we can conclude as in the even case. This achieves the proof of Theorem 4.1 .

\subsection{The class module of the $P$ th cyclotomic function field.}

Let $P$ be a monic irreducible element in $A$ of degree d. Recall that $\lambda_{P}=$ $\exp _{C}\left(\frac{\widetilde{\pi}}{P}\right)$. Let $L=K\left(\lambda_{P}\right)$, recall that $L / K$ is a finite abelian extension of degree $q^{d}-1$ which is unramified outside $P$ and $\infty$ ([25], Proposition 12.7). Let's set $\Delta=$ $\Delta_{P}=\operatorname{Gal}(L / K)$. Let $O_{L}$ be the integral closure of $A$ in $L$, one can show that $O_{L}=$ $A\left[\lambda_{P}\right]$ ([25], Proposition 12.9) and that $P O_{L}=\lambda_{P}^{q^{d}-1} O_{L}$ ([25], Proposition 12.7). Recall that Taelman's class module associated to $L / K$ and the Carlitz module is defined by:

$$
H=H\left(C / O_{L}\right)=\frac{L_{\infty}}{O_{L}+\exp _{C}\left(L_{\infty}\right)},
$$

where $L_{\infty}=L \otimes_{K} K_{\infty}$. We observe that $H$ is a finite $A[\Delta]$-module.

Let $A_{P}$ be the $P$-adic completion of $A$, and set:

$$
X=H \otimes_{A} A_{P} .
$$

Then $X$ is a finite $A_{P}[\Delta]$-module. For $\chi \in \widehat{\Delta}:=\operatorname{Hom}\left(\Delta, A_{P}^{\times}\right)$, we set:

$$
X(\chi)=e_{\chi} X
$$

where $e_{\chi}=\frac{1}{|\Delta|} \sum_{\delta \in \Delta} \chi(\delta) \delta^{-1} \in \mathbb{F}_{q^{d}}[\Delta]$.

If $\chi$ is an even Dirichlet character, then by [7], Theorem 9.12, we have:

$$
L_{P}(1, \chi) X(\chi)=\{0\} .
$$

Furthermore, by [8], Theorem 1, and [16] (see also [3]), if the character $\chi$ is odd and $v_{P}\left(L_{P}^{(1)}(1, \chi)\right)=0$, then $X(\chi)$ is a cyclic $A_{P}$-module. In fact, we have:

Theorem 4.3. If $\chi$ is an odd Dirichlet character of prime conductor $P$, then $L_{P}^{(1)}(1, \chi) X(\chi)$ is a cyclic $A_{P}$-module.

Proof.

If $\chi$ is of type 1 , by [7, Theorem 8.7, we have that $X(\chi)=\{0\}$. Thus we can assume that $\chi$ is of type $n \geq q, n \equiv 1(\bmod q-1)$. Let $\eta_{1}, \ldots, \eta_{n} \in \mathbb{F}_{q^{d}}$ such that:

$$
\forall a \in A, \chi(a)=a\left(\eta_{1}\right) \cdots a\left(\eta_{n}\right) .
$$

Set $\exp _{C}^{(1)}=\sum_{j \geq 1} \frac{j}{D_{j}} \tau^{j}$. As in Corollary 3.5, combining the isomorphism of $A[\Delta]$-modules given by Proposition 2.6 with the evaluation at $z=1$ we obtain a morphism of $A[\Delta]$-modules induced by $\exp _{C}^{(1)}$ :

$$
\psi: \frac{U\left(C / O_{L}\right)}{U_{S t}\left(C / O_{L}\right)} \rightarrow H\left(C / O_{L}\right)
$$

We will first investigate $\left[e_{[\chi]} \operatorname{Coker} \psi\right]_{A[\Delta]}$.

We will intensively use the results obtained in section 3.3.3 Let $\varphi$ be the canonical deformation of the Carlitz module over $\mathbb{T}_{n}\left(K_{\infty}\right)$ (see section 3.3). Let $e v_{\chi}: \mathbb{T}_{n}\left(K_{\infty}\right) \rightarrow \mathbb{F}_{q}\left(\eta_{1}, \ldots, \eta_{n}\right)\left(\left(\frac{1}{\theta}\right)\right)$ be the surjective map given by $e v_{\chi}(f)=$ $\left.f\right|_{t_{1}=\eta_{1}, \ldots, t_{n}=\eta_{n}}$. Set:

$$
\Omega=L_{\infty} \otimes_{\mathbb{F}_{q}} \mathbb{F}_{q}\left(\eta_{1}, \ldots, \eta_{n}\right)
$$


Let $\sigma=\tau \otimes 1: \Omega \rightarrow \Omega$. Then:

$$
e_{\chi} \Omega=g(\chi) \mathbb{F}_{q}\left(\eta_{1}, \ldots, \eta_{n}\right)\left(\left(\frac{1}{\theta}\right)\right) .
$$

Then, by formula (14), we get:

$$
\begin{aligned}
& \forall f \in \mathbb{T}_{n}\left(K_{\infty}\right),(\sigma+\theta)\left(g(\chi) e v_{\chi}(f)\right)=g(\chi) e v_{\chi}\left(\varphi_{\theta}(f)\right) . \\
& \forall f \in \mathbb{T}_{n}\left(K_{\infty}\right), \exp _{C, \sigma}\left(g(\chi) e v_{\chi}(f)\right)=g(\chi) e v_{\chi}\left(\exp _{\varphi}(f)\right),
\end{aligned}
$$

where:

$$
\exp _{C, \sigma}=\sum_{j \geq 0} \frac{1}{D_{j}} \sigma^{j}
$$

Now, by Theorem 2.13, we have:

$$
e_{\chi} U_{S t}\left(C / O_{L}\right) \otimes_{\mathbb{F}_{q}} \mathbb{F}_{q}\left(\eta_{1}, \ldots, \eta_{n}\right)=A\left[\eta_{1}, \ldots, \eta_{n}\right] g(\chi) L(1, \chi),
$$

where $L(1, \chi)=\sum_{a \in A_{+}} \frac{\chi(a)}{a} \in \mathbb{F}_{q}\left(\eta_{1}, \ldots, \eta_{n}\right)\left(\left(\frac{1}{\theta}\right)\right)^{\times}$. Thus:

$$
e_{\chi} U_{S t}\left(C / O_{L}\right) \otimes_{\mathbb{F}_{q}} \mathbb{F}_{q}\left(\eta_{1}, \ldots, \eta_{n}\right)=g(\chi) e v_{\chi}\left(U_{S t}\left(\varphi / A\left[t_{1}, \ldots, t_{n}\right]\right)\right) .
$$

Select $v$ a place of $L$ above $\infty$, and let $\tilde{\pi}_{v}=(0, \ldots, O, \widetilde{\pi}, 0, \ldots, 0) \in L_{\infty}=$ $\prod_{w \in S_{\infty}(L)} L_{w}$ which has zero at all its components except at the component $v$. Let's set:

$$
\tilde{\pi}_{\chi}=e_{\chi} \tilde{\pi}_{v} \in e_{\chi} \Omega
$$

Then by [7, Proposition 8.4, we have:

$$
\operatorname{Ker}\left(\left.\exp _{C, \sigma}\right|_{e_{\chi} \Omega}\right)=\widetilde{\pi}_{\chi} A\left[\eta_{1}, \ldots, \eta_{n}\right] .
$$

Since $\chi$ is odd and of type $>1$, we deduce that:

$$
e_{\chi} U\left(C / O_{L}\right) \otimes_{\mathbb{F}_{q}} \mathbb{F}_{q}\left(\eta_{1}, \ldots, \eta_{n}\right)=\tilde{\pi}_{\chi} A\left[\eta_{1}, \ldots, \eta_{n}\right] .
$$

Since $e v_{\chi}\left(\mathbb{B}\left(t_{1}, \ldots, t_{n}\right)\right)=\left[e_{\chi} H\left(C / O_{L}\right) \otimes_{\mathbb{F}_{q}} \mathbb{F}_{q}\left(\eta_{1}, \ldots, \eta_{n}\right)\right]_{A\left[\eta_{1}, \ldots, \eta_{n}\right]}$ ([5, Corollary 9.3), by Lemma 3.1 and [7, Theorem A, Theorem B and Proposition 8.5, we get:

$$
e_{\chi} U\left(C / O_{L}\right) \otimes_{\mathbb{F}_{q}} \mathbb{F}_{q}\left(\eta_{1}, \ldots, \eta_{n}\right)=g(\chi) e v_{\chi}\left(U\left(\varphi / A\left[t_{1}, \ldots, t_{n}\right]\right)\right) .
$$

Set:

$$
\exp _{C, \sigma}^{(1)}=\sum_{j \geq 1} \frac{j}{D_{j}} \sigma^{j}
$$

As in the case of section 3.3.3. $\exp _{C, \sigma}^{(1)}$ induces a map:

$$
\frac{e_{\chi} \Omega}{g(\chi) \mathcal{N}} \rightarrow \frac{e_{\chi} \Omega}{g(\chi) \mathcal{N}}
$$

where

$$
\mathcal{N}=\left\{x \in \mathbb{F}_{q}\left(\eta_{1}, \ldots, \eta_{n}\right)\left(\left(\frac{1}{\theta}\right)\right), v_{\infty}(x)>\frac{n-q}{q-1}\right\}
$$

(note that $e_{\chi} \Omega=\widetilde{\pi} A\left[\eta_{1}, \ldots, \eta_{n}\right] \oplus g(\chi) \mathcal{N}$ ). Now, using $e v_{\chi}$, the proof of Proposition 3.14 works in this case and the above map is injective.

Observe that:

$$
\begin{gathered}
\exp _{C, \sigma}\left(e_{\chi} \Omega\right)=g(\chi) \mathcal{N} \\
e_{\chi} \Omega=\widetilde{\pi}_{\chi} A\left[\eta_{1}, \ldots, \eta_{n}\right] \oplus g(\chi) \mathcal{N} .
\end{gathered}
$$


Set $V=\left\{x \in e_{\chi} \Omega, \exp _{C, \sigma}^{(1)}(x) \in g(\chi) A\left[\eta_{1}, \ldots, \eta_{n}\right]+g(\chi) \mathcal{N}\right\}$. Then, we have an injective map induced by $\exp _{C, \sigma}^{(1)}$ :

$$
\frac{V}{g(\chi) \mathcal{N}} \hookrightarrow \frac{g(\chi) A\left[\eta_{1}, \ldots, \eta_{n}\right] \oplus g(\chi) \mathcal{N}}{g(\chi) \mathcal{N}}
$$

Note that the map $\psi$ defined in (16) induces a map of $A\left[\eta_{1}, \ldots, \eta_{n}\right]$-modules:

$$
\psi_{\chi}: e_{\chi} \frac{U\left(C / O_{L}\right)}{U_{S t}\left(C / O_{L}\right)} \otimes_{\mathbb{F}_{q}} \mathbb{F}_{q}\left(\eta_{1}, \ldots, \eta_{n}\right) \rightarrow \frac{e_{\chi} \Omega}{g(\chi) A\left[\eta_{1}, \ldots, \eta_{n}\right] \oplus g(\chi) \mathcal{N}} .
$$

If we consider the proof of Theorem 3.17 there exists a monic polynomial $G \in$ $A\left[\eta_{1}, \ldots, \eta_{n}\right]$ such that:

$$
\begin{gathered}
e_{\chi} U_{S t}\left(C / O_{L}\right) \otimes_{\mathbb{F}_{q}} \mathbb{F}_{q}\left(\eta_{1}, \ldots, \eta_{n}\right) \subset G \widetilde{\pi}_{\chi} A\left[\eta_{1}, \ldots, \eta_{n}\right] \\
\operatorname{Ker} \psi_{\chi}=\frac{G \widetilde{\pi}_{\chi} A\left[\eta_{1}, \ldots, \eta_{n}\right]}{e_{\chi} U_{S t}\left(C / O_{L}\right) \otimes_{\mathbb{F}_{q}} \mathbb{F}_{q}\left(\eta_{1}, \ldots, \eta_{n}\right)}, \\
G \widetilde{\pi}_{\chi} A\left[\eta_{1}, \ldots, \eta_{n}\right]+g(\chi) \mathcal{N}=V .
\end{gathered}
$$

Let $e_{\chi} \mathcal{M}=g(\chi) e v_{\chi}\left(\mathcal{M}\left(\varphi / A\left[t_{1}, \ldots, t_{n}\right]\right)\right)$. Then we have a natural injective map of $A\left[\eta_{1}, \ldots, \eta_{n}\right]$-modules (induced by $\left.\exp _{C, \sigma}^{(1)}\right)$ :

$$
\begin{gathered}
\frac{G \widetilde{\pi}_{\chi} A\left[\eta_{1}, \ldots, \eta_{n}\right] \oplus g(\chi) \mathcal{N}}{e_{\chi} U_{S t}\left(C / O_{L}\right) \otimes_{\mathbb{F}_{q}} \mathbb{F}_{q}\left(\eta_{1}, \ldots, \eta_{n}\right) \oplus g(\chi) \mathcal{N}} \longleftrightarrow \frac{g(\chi) A\left[\eta_{1}, \ldots, \eta_{n}\right] \oplus g(\chi) \mathcal{N}}{e_{\chi} \mathcal{M} \oplus_{\gamma} g(\chi) \mathcal{N}} \\
\frac{g(\chi) A\left[\eta_{1}, \ldots, \eta_{n}\right]}{e_{\chi} \mathcal{M}}
\end{gathered}
$$

thus:

$$
\operatorname{Ker} \psi_{\chi} \hookrightarrow \frac{g(\chi) A\left[\eta_{1}, \ldots, \eta_{n}\right]}{e_{\chi} \mathcal{M}}
$$

Let $\mathcal{M}$ be the $A[\Delta]$-module via $C$ generated by $u_{[\chi]}^{(1)}(1)$ (see section 4.2) and let $\sqrt{\mathcal{M}}=\left\{x \in O_{L}, \exists a \in A \backslash\{0\}, C_{a}(x) \in \mathcal{M}\right\}$. Let's consider the map induced by $\psi$ :

$$
\psi_{[\chi]}: e_{[\chi]} \frac{U\left(C / O_{L}\right)}{U_{S t}\left(C / O_{L}\right)} \rightarrow e_{[\chi]} H\left(C / O_{L}\right)
$$

Then, by the above discussion, we have an injective morphism of $A[\Delta]$-modules:

$$
\operatorname{Ker} \psi_{[\chi]} \hookrightarrow \frac{e_{[\chi]} O_{L}}{\mathcal{M}} .
$$

But $\operatorname{Ker} \psi_{[\chi]}$ is a finite $A$-module, therefore we have an injective morphism of $A[\Delta]$ modules:

$$
\operatorname{Ker} \psi_{[\chi]} \hookrightarrow \frac{\sqrt{\mathcal{M}}}{\mathcal{M}}
$$

Note that $\mathcal{M}$ is a free $A$-module of rank $|[\chi]|$. Thus, by $\left[22, \frac{\sqrt{\mathcal{M}}}{\mathcal{M}}\right.$ is a finite $A[\Delta]$-module. By Corollary 2.12, we have:

$$
\left[e_{[\chi]} \frac{U\left(C / O_{L}\right)}{U_{S t}\left(C / O_{L}\right)}\right]_{A[\Delta]}=\left[e_{[\chi]} H\left(C / O_{L}\right)\right]_{A[\Delta]} .
$$

Thus:

$$
\left[\operatorname{Ker} \psi_{[\chi]}\right]_{A[\Delta]}=\left[\operatorname{Coker} \psi_{[\chi]}\right]_{A[\Delta]}
$$


Since $\frac{U\left(C / O_{L}\right)}{U_{S t}\left(C / O_{L}\right)}$ is $A[\Delta]$-cyclic, we have $\left[\operatorname{Ker} \psi_{[\chi]}\right]_{A[\Delta]} e_{[\chi]} H\left(C / O_{L}\right)$ is $A[\Delta]$-cyclic. But $\left[\operatorname{Ker} \psi_{[\chi]}\right]_{A[\Delta]}$ divides $\left[\frac{\sqrt{\mathcal{M}}}{\mathcal{M}}\right]_{A[\Delta]}$ in $A[\Delta]$, thus we deduce that the module $\left[\frac{\sqrt{\mathcal{M}}}{\mathcal{M}}\right]_{A[\Delta]} e_{[\chi]} H\left(C / O_{L}\right)$ is $A[\Delta]$-cyclic. This in particular implies that :

$$
\left(\operatorname{Fitt}_{A_{P}} e_{\chi}\left(\frac{\sqrt{\mathcal{M}}}{\mathcal{M}} \otimes_{A} A_{P}\right)\right) X(\chi) \text { is } A_{P}-\text { cyclic, }
$$

where for $M$ a finite $A_{P}$-module, Fitt $_{A_{P}} M$ denotes its Fitting ideal.

Let $F$ be the $P$-adic completion of $L$. Let $O_{F}$ be the valuation ring of $F$ and let $\mathfrak{P}=\left\{x \in F, v_{P}(x)>0\right\}$. Recall from section 3.4 that $C$ extends into a morphism of $\mathbb{F}_{q}$-algebras $C: A_{P} \rightarrow A_{P}\{\{\tau\}\}$. We denote by $C(\mathfrak{P})$ the $\mathbb{F}_{q}$-vector space $\mathfrak{P}$ viewed as an $A_{P}$-module via $C$. Then $\log _{C, P}$ induces an isomorphism of $A_{P}[\Delta]$-modules:

$$
\log _{C, P}: C\left(\mathfrak{P}^{2}\right) \simeq \mathfrak{P}^{2} .
$$

We also observe that, since $\chi$ is odd of type $>1, g\left(\chi^{q^{j}}\right) A_{P}=e_{\chi^{q^{j}}} O_{F} \subset \mathfrak{P}^{2}$ for all $j \geq 0$ (this follows for example from [29], Theorem III). In particular:

$$
\mathcal{M}, \sqrt{\mathcal{M}} \subset C\left(\mathfrak{P}^{2}\right) .
$$

Let $\overline{\mathcal{M}}$ be the $P$-adic closure of $\mathcal{M}$ in $C\left(\mathfrak{P}^{2}\right)$. Then clearly $\overline{\mathcal{M}}$ is the $A_{P}[\Delta]$-module generated by $u_{[\chi]}^{(1)}(1)$. Now, by Proposition 4.2 , we have:

$$
\log _{C, P}\left(e_{\chi} \overline{\mathcal{M}}\right)=g(\chi) L_{P}^{(1)}(1, \chi) A_{P} .
$$

By Theorem 4.1, we have an isomorphism of $A_{P}$-modules:

$$
e_{\chi}\left(\mathcal{M} \otimes_{A} A_{P}\right) \simeq e_{\chi} \overline{\mathcal{M}} \text {. }
$$

Furthermore:

$$
\operatorname{Fitt}_{A_{P}} \frac{e_{\chi} C\left(\mathfrak{P}^{2}\right)}{e_{\chi} \overline{\mathcal{M}}}=L_{P}^{(1)}(1, \chi) A_{P} .
$$

Let $\overline{\sqrt{\mathcal{M}}}$ be the $P$-adic closure of $\sqrt{\mathcal{M}}$ in $C\left(\mathfrak{P}^{2}\right)$. Then, we have an isomorphism of $A_{P}$-modules:

Thus:

$$
e_{\chi}\left(\frac{\sqrt{\mathcal{M}}}{\mathcal{M}} \otimes_{A} A_{P}\right) \simeq e_{\chi} \frac{\overline{\sqrt{\mathcal{M}}}}{\overline{\mathcal{M}}} .
$$

$$
L_{P}^{(1)}(1, \chi) A_{P} \subset \operatorname{Fitt}_{A_{P}} e_{\chi}\left(\frac{\sqrt{\mathcal{M}}}{\mathcal{M}} \otimes_{A} A_{P}\right) .
$$

The Theorem follows.

Remark 4.4. Let $P$ be a monic irreducible element in $A$ of degree d. Note that, in general, for $\chi$ odd of conductor $P$, we have:

$$
L_{P}^{(1)}(1, \chi) X(\chi) \neq\{0\} .
$$

Indeed, let $\chi_{P}$ be the $P$-adic Teichmüller character (observe that this is a Dirichlet character of type 1), i.e. :

$$
\forall a \in A, \chi_{P}(a) \equiv a \quad\left(\bmod P A_{P}\right) .
$$

Let $n \in\left\{2, \ldots, q^{d}-2\right\}, n \equiv 1(\bmod q-1)$. Then, by [28], we have:

$$
X\left(\chi_{P}^{n}\right) \neq\{0\} \text { if and only if } B C\left(q^{d}-n\right) \equiv 0 \quad(\bmod P),
$$


BRUNO ANGLÈS AND FLORIC TAVARES RIBEIRO

where $B C\left(q^{d}-n\right)$ denotes the $\left(q^{d}-n\right)$ th Bernoulli-Carlitz number (see 13], paragraph 9.2). Let $\beta(n-1)$ be the $(n-1)$ th Bernoulli-Goss number (see [14], 16], [18), then:

$$
L_{P}^{(1)}\left(1, \chi_{P}^{n}\right) \equiv \beta(n-1) \quad(\bmod P) .
$$

For example, for $q=3, P=\theta^{3}-\theta-1$, we have (by [13], page 354):

$$
B C(10)=\frac{2 \theta^{6}+2 \theta^{4}+2 \theta^{2}+1}{\theta^{3}+2 \theta} \equiv 0 \quad(\bmod P),
$$

and a direct computation shows:

$$
\beta(16)=\theta^{30}+2 \theta^{28}+2 \theta^{4}+\theta^{2}+1 \equiv 1 \quad(\bmod P) .
$$

This implies:

$$
L_{P}^{(1)}\left(1, \chi_{P}^{17}\right) X\left(\chi_{P}^{17}\right) \neq\{0\}
$$

\section{REFERENCES}

[1] G. Anderson, Log-algebraicity of twisted $A$-harmonic series and special values of $L$-series in characteristic $p$, Journal of Number Theory 60 (1996), 165-209.

[2] G. Anderson, D. Thakur, Tensor powers of the Carlitz module and zeta values, Annals of Mathematics 132 (1990), 159-191.

[3] B. Anglès, A. Bandini, F. Bars, I. Longhi, Iwasawa Main Conjecture for the Carlitz cyclotomic extension and applications, arXiv : 1412.5957 .

[4] B. Anglès, F. Pellarin, Universal Gauss-Thakur sums and L-series, Inventiones mathematicae 200 (2015), 653-669.

[5] B. Anglès, F. Pellarin, F. Tavares Ribeiro, Arithmetic of positive characteristic $L$-series values in Tate algebras, to appear in Compositio Mathematica, arXiv:1402.0120

[6] B. Anglès, F. Pellarin, F. Tavares Ribeiro, Anderson-Stark units for $\mathbb{F}_{q}[\theta]$, arXiv: 1501.06804 .

[7] B. Anglès, L. Taelman, Arithmetic of characteristic $p$ special $L$-values, Proceedings of the London Mathematical Society 110 (2015), 1000-1032.

[8] B. Anglès, L. Taelman, The Spiegelungssatz for the Carlitz module, Journal of Number Theory 133 (2013), 2139-2142.

[9] F. Demeslay, A class formula for $L$-series in positive characteristic, arXiv : 412.3704v1 .

[10] J. Fang, Special L-values of abelian t-modules, Journal of Number Theory 147 (2015), 300325.

[11] J. Fang, Equivariant $L$-values for abelian $t$-modules, arXiv: $1503.07243 v 1$.

[12] J. Fresnel, M. van der Put, Rigid Analytic Geometry and Its Applications, Birkhäuser, 2004.

[13] D. Goss, Basic Structures of Function Field Arithmetic, Springer, Berlin, 1996.

[14] D. Goss, Units and class groups in the arithmetic of function fields, Bulletin of the American Mathematical Society 13 (1985), 131-132.

[15] D. Goss, v-adic Zeta Functions, L-series and Measures for Function Fields, Inventiones mathematicae 55 (1979), 107-116.

[16] D. Goss, W. Sinnott, Class groups of function fields, Duke Mathematical Journal 52 ( 1985), 507-516.

[17] R. Greenberg, Iwasawa theory-past and present, Advanced Studies in Pure Mathematics 30 (2001), 335-385.

[18] K. Ireland, D. Small, A note on Bernoulli-Goss polynomials, Canadian Mathematical Bulletin 27 (1984), 179-184.

[19] S. Lang, Cyclotomic Field I and II, Springer-Verlag, 1990.

[20] H.W. Leopoldt, Zur Strukture der l-Klassengruppe galoischer Zahlkorper, Journal für die reine und angewandte Mathematik 199 (1958), 165-174.

[21] B. Mazur, A. Wiles, Class fields of abelian extensions of $\mathbb{Q}$, Inventiones mathematicae 76 (1984), 179-330.

[22] B. Poonen, Local height functions and the Mordell-Weil theorem for Drinfeld modules, Compositio Mathematica 97 (1995), 349-368.

[23] F. Pellarin, Values of certain $L$-series in positive characteristic, Annals of Mathematics 176 (2012), 1-39. 
[24] E. Noether, Normalbasis bei Körpern ohne höhere Verzweigung, Journal für die reine und angewandte Mathematik 167 (1931), 147-152.

[25] M. Rosen, Number Theory in Function Fields, Springer, 2002.

[26] L. Taelman, A Dirichlet unit theorem for Drinfeld modules, Mathematische Annalen 348 (2010), 899-907.

[27] L. Taelman, Special L-values of Drinfeld modules, Annals of Mathematics 75 (2012), 369-391.

[28] L. Taelman, A Herbrand-Ribet theorem for function fields, Inventiones mathematicae 188 (2012), 253-275.

[29] D. Thakur, Gauss sums for $\mathbb{F}_{q}[t]$, Inventiones mathematicae 94 (1988), 105-112 .

[30] L. C. Washington, Introduction to Cyclotomic Fields, Second Edition, Springer, 1997.

Université de Caen Basse-Normandie, Laboratoire de Mathématiques Nicolas Oresme, CNRS UMR 6139, Campus II, Boulevard Maréchal Juin, B.P. 5186, 14032 Caen Cedex, FRANCE.

E-mail address: bruno.angles@unicaen.fr, floric.tavares-ribeiro@unicaen.fr 